\title{
Complex and Multidimensional Lipid Raft Alterations in a Murine Model of Alzheimer's Disease
}

\author{
Wayne Chadwick, ${ }^{1}$ Randall Brenneman, ${ }^{1,2}$ Bronwen Martin, ${ }^{3}$ and Stuart Maudsley ${ }^{1}$ \\ ${ }^{1}$ Receptor Pharmacology Unit, National Institute on Aging, National Institutes of Health, 251 Bayview Boulevard, \\ Suite 100, Baltimore, MD 21224, USA \\ ${ }^{2}$ Miller School of Medicine, University of Miami, Miami, FL 33124, USA \\ ${ }^{3}$ Metabolism Unit, National Institute on Aging, National Institutes of Health, 251 Bayview Boulevard, \\ Suite 100, Baltimore, MD 21224, USA
}

Correspondence should be addressed to Stuart Maudsley, maudsleyst@mail.nih.gov

Received 17 May 2010; Accepted 27 July 2010

Academic Editor: Gemma Casadesus

Copyright ( $) 2010$ Wayne Chadwick et al. This is an open access article distributed under the Creative Commons Attribution License, which permits unrestricted use, distribution, and reproduction in any medium, provided the original work is properly cited.

\begin{abstract}
Various animal models of Alzheimer's disease (AD) have been created to assist our appreciation of AD pathophysiology, as well as aid development of novel therapeutic strategies. Despite the discovery of mutated proteins that predict the development of AD, there are likely to be many other proteins also involved in this disorder. Complex physiological processes are mediated by coherent interactions of clusters of functionally related proteins. Synaptic dysfunction is one of the hallmarks of AD. Synaptic proteins are organized into multiprotein complexes in high-density membrane structures, known as lipid rafts. These microdomains enable coherent clustering of synergistic signaling proteins. We have used mass analytical techniques and multiple bioinformatic approaches to better appreciate the intricate interactions of these multifunctional proteins in the 3xTgAD murine model of AD. Our results show that there are significant alterations in numerous receptor/cell signaling proteins in cortical lipid rafts isolated from $3 x \operatorname{TgAD}$ mice.
\end{abstract}

\section{Introduction}

Alzheimer's disease (AD) is one of the most prevalent neurodegenerative disorders amongst adults of advanced age, and it is the most common form of dementia and cognitive impairment $[1,2]$. The behavioral abnormalities in AD result from dysfunction and death of neurons in brain regions involved in cognition and mood, such as the hippocampus, amygdala, and cortical regions. Progressive short-term and eventual long-term memory loss and reduced cognitive capacity are associated with two primary neurodegenerative lesions, that is, extra- and intracellular $\beta$-amyloid plaques, as well as neurofibrillary tangles (NFTs) composed of the microtubule protein tau [3-5]. In addition to the effects of amyloid plaques and NFTs, the lipid trafficking molecule, apolipoprotein E4 (apoE4), has also been demonstrated to be a genetic risk factor for $\mathrm{AD}[6,7]$. The $\mathrm{AD}$ characteristic extracellular plaques, found in both the hippocampus and cortex of AD patients, consist of 39-42 amino acid long amyloid- $\beta$ (A $\beta)$ peptides. These extracellular peptides are generated by digestion of a transmembrane amyloid precursor protein (APP). Proteolysis of the transmembrane APP by a set of intramembrane enzymes, $\beta$ - (also known as BACE$1)$ and $\gamma$-secretases, is thought to be responsible for toxic $\mathrm{A} \beta$ creation [5]. The discovery of familial mutations in the APP gene that were strongly correlated with the presentation of $\mathrm{AD}$ reinforced the importance of $\mathrm{A} \beta$ processing in this disorder. A growing body of evidence indicates that changes in lipid and cholesterol homeostasis can influence $\mathrm{AD}$ progression and specifically $\mathrm{A} \beta$ production. One of the prime sub cellular regions of amyloidogenic APP processing is thought to be cholesterol-enriched membrane microdomains, termed lipid rafts [8]. Cellular organization of protein signaling complexes, to enhance the magnitude and fidelity of transmembrane signaling receptors, is facilitated by variations in the lipid constituents of the plasma membrane. Lipid rafts represent discontinuous regions of the plasma membrane that form functional microdomains, 
which constrain the association of proteins in a coherent and advantageous manner with respect to neurotransmissive signaling [9]. Disruption of the correct stoichiometry of signaling complexes within lipid rafts may underpin the etiology of many different neurodegenerative disorders [1012]. The hypothesis that changes in the lipid composition of rafts contribute to $\mathrm{AD}$ pathology has gained considerable support. For example, ApoE4 has been strongly correlated with the generation of $\mathrm{AD}$ symptomatology. Both of the amyloidogenic processing enzymes ( $\beta$ - and $\gamma$-secretase), as well as APP, are all enriched in lipid raft membranes $[13,14]$. Reinforcing the connection between lipid density levels and $\mathrm{A} \beta$ production, increasing cholesterol levels elevate the activity of both $\beta$-secretase (BACE-1) and $\gamma$-secretase $[14,15]$. In addition, ganglioside lipids, which are also enriched in lipid rafts, can control the assembly of amyloid$\beta$ proteins [16, 17]. Changes in ganglioside composition, similar to those noted in human $\mathrm{AD}$ patients, are also observed in different transgenic mouse models of AD [18]. In addition to a role of the lipid components of lipid rafts in controlling amyloidogenesis, these raft environments may also affect NFTs as well. It has been demonstrated that $\mathrm{A} \beta$ can induce activation of the tyrosine kinase Fyn in neuronal cells, that is then recruited to lipid rafts which catalyzes phosphorylation of tyrosine residue 18 on tau [19, 20]. Association of $\mathrm{A} \beta$ plaques to lipid rafts can mediate recruitment of excess Fyn to the rafts, as well as further recruitment and phosphorylation of tau. These activities are thought to induce neurotoxicity via the effects of tau-induced changes in the actin cytoskeleton and receptor/cellular signaling pathways [21]. Therefore, the potential changes in the lipid composition of lipid rafts, caused by exposure to cytotoxic activities characteristic to $\mathrm{AD}$, can induce profound changes in cellular signal transduction and thereby induce intracellular changes that lead to the development of $\mathrm{AD}$. The complexity of protein complexes within the lipid raft environments raises considerable challenges to understanding the molecular mechanisms of $\mathrm{AD}$ pathophysiology in both the hippocampus and cortex of animals. Therefore, we have employed a shotgun proteomics approach, allied to advanced bioinformatic functional profiling, to gain a broad and detailed appreciation of the alterations in signaling proteins in lipid rafts in the triple-transgenic (3xTgAD) model of AD [22]. Our study demonstrates that cortical lipid rafts are profoundly affected in the $3 \times \mathrm{TgAD}$ mice and that many of the neurophysiological deficits characteristic of AD (impaired synaptic strength, impaired learning and memory, and increased oxidative stress) can be strongly linked to changes in receptor and cell signaling events in the lipid rafts in these animals. Therefore, the lipid raft environments can be seen as one of the most important pathophysiological loci of this disorder.

\section{Methods}

2.1. Animals and Morris Water Maze Testing. Animal care and experimental procedures followed NIH guidelines and were approved by the National Institute on Aging Animal Care, and Use Committee. Experiments were performed using male 3xTgAD [22-24] and control male C57-BL6 mice that were maintained under a 12-hour light/12-hour dark cycle with continuous access to food and water. Water maze testing took place using a modified version of the methodology described previously [25]. Briefly, animals $(n=$ 10 per group, control male C57-BL6 or male 3xTgAD, on a C57-BL6 background) received 8 days of acquisition training using a nonvisible target platform, consisting of four trials per day, with an intertrial interval of approximately 10 minutes. Each trial lasted until the animal found the platform, or for a maximum of 60 seconds; animals that failed to find the platform within 60 seconds were guided there by the experimenter. On each trial, mice were placed into the pool, facing the wall, with start locations varied pseudorandomly. Distance swam to escape the water, escape time, and swim speed were measured for either control or 3xTgAD mice using a HVS2020 automated tracking system (HVS Image, UK).

\subsection{Isolation of Lipid Raft Detergent-Resistant Membranes.} The mice were anesthetized with isoflurane, decapitated, and the brain was microdissected on ice. After removal of the cortex, the tissue was split into left and right hemisphere, half for mass spectrometry raft analysis and half to prepare lipid raft tissues for Western blot analysis. The hemicortices were washed twice in ice-cold phosphate buffered saline (PBS) and then transferred into a Tris-saline buffer supplemented with a cocktail of protease and phosphatase inhibitors $(50 \mathrm{mM}$ Tris- $\mathrm{HCl}, 150 \mathrm{mM} \mathrm{NaCl}, 5 \mathrm{mM}$ EDTA, and Roche CompleteMini (Roche Diagnostics Inc.) protease and phosphatase inhibitor cocktail, pH 7.4). Crude tissue disruption was then rapidly achieved (at $4^{\circ} \mathrm{C}$ ) using a sonic dismembrator (Fisher Scientific Model 100) followed by a brief centrifugation $\left(4^{\circ} \mathrm{C}\right.$, $1000 \times$ g, 10 minutes) to pellet cell nuclei and unbroken cells. The resultant supernatant was removed and Triton X-100 (Sigma Aldrich, USA) was added to the Tris-saline buffer to a final concentration of $1 \%$. The supernatant membranes were then incubated at $4^{\circ} \mathrm{C}$ for 60 minutes in the Triton $\mathrm{X}-100$ Tris-saline solution. After incubation, the supernatant solution was then added to a discontinuous gradient of $30 \%$ and 60\% OptiPrep (Iodixanol, Sigma Aldrich, and U.S.A.) before centrifugation at $200000 \times \mathrm{g}$ for 16 hours at $4^{\circ} \mathrm{C}$. After centrifugation, a detergent-resistant lipid band was evident in the vertical solution column. Multiple fractions of $300 \mu \mathrm{l} \mathrm{vol-}$ umes were then removed from the vertical centrifugation column. Proteins were then extracted from these fractions using a proprietary ProteoExtract (EMD Biosciences) kit, according to the manufacturer's instructions. Isolated protein pellets were then prepared for mass spectrometric analysis.

2.3. Mass Spectrometric Protein Analysis. Protein pellets were dissolved into an ammonium bicarbonate buffer (100 mM, pH 8.5) and then reduced with dithiothreitol (500 mM: Pierce Biotechnology), alkylated with iodoacetamide ( $800 \mathrm{mM}$ : Sigma Aldrich) and then digested with modified trypsin (5-10 $\mu$ g) (Promega) at $37^{\circ} \mathrm{C}$ for 17 hours. Proteolysis was terminated by the addition of glacial acetic acid. Tryptic peptides were then loaded onto a desalting 
column $(360 \times 200 \mu \mathrm{m}$ fused silica packed with $15 \mathrm{~cm}$ of C18 beads (YMC ODS-AQ, Waters)), washed with $0.1 \%$ acetic acid and eluted into sample tubes with $80 \%$ acetonitrile in $0.1 \%$ acetic acid. Sample volume was reduced to usable volumes under vacuum on a Savant SpeedVac. Samples were then transferred onto a PicoFrit $(75 \times 100 \mathrm{~mm})$ column packed with ProteoPep II $\mathrm{C}_{18}, 300 \AA, 5 \mu \mathrm{m}$ particles (New Objective) connected to a nanoliquid chromatography system (Dionex, Sunnyvale, CA) online with an LTQ ion trap mass spectrometer (Thermo Finnigan, San Jose, CA). The peptides were eluted using a linear gradient of $0-65 \%$ acetonitrile over 90 minutes at a flow rate of $250 \mathrm{nl} / \mathrm{min}$ directly into the mass spectrometer, which was operated to generate collision-induced dissociation spectra (data-dependent MS/MS mode). The resultant tandem mass spectrometry data were processed using the BioWorks suite, and multiple collected spectra were used to interrogate the NCBI nonredundant mouse and Swiss-Prot protein sequence databases, using the computer algorithm SEQUEST to generate accurate protein identities. Protein genpept accession identities were then converted to Official Gene Symbol terms using NIAID-DAVID v. 6.7 (http://david.abcc.ncifcrf.gov/). The statistical analysis and validation of the search results were performed using MASCOT (Matrix Science). For protein identification, a maximum of three missed tryptic cleavages was used, including fixed modification of carbamidomethylation and variable modifications of oxidized methionine and $\mathrm{N}$-terminal glutamine conversion to pyroglutamic acid in the search. Only proteins with at least two validated peptides and a total score 25 and a confidence of identification of at least $95 \%$ were considered valid for reporting. Where required, additional spectral counting was performed to determine simplistic relative quantitation in conjunction with the reported number of identified unambiguous peptides per protein. Three lipid raft fraction samples (fractions 2, 3, and 4) from each three control (nontransgenic gender/age matched C57-BL6) or Alzheimer's disease (3xTgAD) were pooled and then run in an individual random order. Proteins identified based on two unambiguous peptides that were present in at least two out of the three individual animals were employed for further expression pattern analysis.

2.4. Lipid Raft Band Quantification. Digitized images of centrifugal vertical fluid columns were obtained using a Canon Digital camera and were converted from Joint Photographic Experts Group (JPG) files to a TIFF (Tagged Image File Format) form using L-Process v. 2.2 (image handling software: Fuji-Film). Image densitometry was then performed using Fuji-Film Image Gauge v. 4.2. Lipid raft band intensity was represented as a relative absorbance unit $(\mathrm{AU})$ value with background $(\mathrm{B})$ subtraction per square pixel $\left(\mathrm{px}^{2}\right)\left(\mathrm{AU}-\mathrm{B} / \mathrm{px}^{2}\right)$.

2.5. Western Blotting Procedures. For the examination of specific proteins in cortical cell samples (both lipid raft and nonlipid raft), aliquots were removed from centrifugal fractions from Section 2.2 and their protein concentration was determined with a standard BCA protocol. Aliquot samples for western blotting analysis were then mixed with an equal volume of Laemmli sample buffer [26]. Samples were resolved using one-dimensional gel electrophoresis (SDS-PAGE), followed by electrotransfer to polyvinylenedifluoride (PVDF: PerkinElmer, Waltham, MA). PVDF membranes were blocked for one hour at room temperature in $4 \%$ nonfat milk (Santa Cruz; Santa Cruz, CA) before application of specific primary antisera in the same nonfat milk. The presence of primary antibody reactivity with the PVDF membrane was detected by the application of a $1: 5000$ dilution of a species-specific alkaline phosphataseconjugated secondary antibody (Sigma, St. Louis, MO). PVDF-bound immune complexes of secondary and primary antibodies were subsequently detected using enzyme-linked chemifluorescence (ECF: GE Healthcare; Pittsburgh, PA). Chemifluorescent signals from the membranes were captured and quantified using a Typhoon 9410 phosphorimager (GE Healthcare, Pittsburgh, PA). Specific primary antisera used were obtained from the following sources: flotillin-1, proline-rich tyrosine kinase 2 (Pyk2), focal adhesion kinase (FAK), G protein-coupled receptor kinase interactor-1 (GIT1 ), and paxilin antibodies were obtained from BD Bioscience, San Jose, CA; Janus kinase 2 (Jak2), v-Crk avian sarcoma virus CT10 oncogene homolog (Crk), and insulin receptor substrate-1 (IRS1) antibodies were obtained from Santa Cruz Biotechnology Corporation, CA; caspase-7, FKBP12rapamycin complex-associated protein 1 /mammalian target of rapamycin (FRAP1/mTOR), and Fyn and IGF-1 receptor beta antibodies were obtained from Cell Signaling Technology, Danvers, MA); G protein-regulated inducer of neurite outgrowth 2 (Grin2) antibody was obtained from Sigma Aldrich. For the identification of nonspecific total proteins in each sample the highly sensitive protein dye, SYPRO Ruby (Invitrogen Corporation) was employed. Fixed SDS-PAGE gels were immersed in SYPRO-Ruby for 1 hour and then washed in deionized water before scanning using a Typhoon 9410 phosphorimager (GE Healthcare, Pittsburgh, PA).

2.6. Bioinformatic Analyses. Protein identities were converted to standard gene symbol nomenclature for simplicity of usage with the batch conversion tool of NIH Bioinformatics Resources DAVID v. 6.7 (http://david.abcc.ncifcrf.gov/). Primary protein sets (containing consistently identified lipid raft extract proteins) were organized into functional signaling pathway groups and then analyzed for their differential significance of population of these canonical signaling pathways. To compare the relative degree of association of specific signaling pathways with the control or $3 \times \mathrm{TgAD}$ protein sets, the difference between the signaling pathways "hybrid scores" was calculated (control subtracted from $3 x \operatorname{TgAD}$ ). The magnitude of the "hybrid score" is indicative of strength and significance of association of the input protein set with the specific signaling pathway. Signaling pathway hybrid scores were generated using a process that takes into account the significant population and potential activation of that pathway by multiplying the pathway enrichment ratio (percentage of proteins in a designated pathway that were also found in the experimental dataset) 
and the probability $(P)$ that the respective pathway is significantly associated with the experimental dataset. However, to create a simple numerical value, the hybrid pathway score is calculated by multiplication of the ratio with the negative $\log -10$ of the $P$ value. Each signaling pathway considered was required to contain at least two unique proteins from either control or 3xTgAD datasets and possess a $P$ value of $\leq .05$. Unbiased network analysis was also performed on subsets of the primary protein sets that were specifically limited to transmembrane receptor proteins. The networks generated create predictions of the most likely functional interactions between proteins in a complex dataset [27]. Networks are created to indicate the most significant series of molecular interactions. The networks with the highest predictive "scores" possess the highest number of statistically significant "focus molecules": "focus molecules" are proteins that are present in the most statistically-likely predicted functional network and are present in the input experimental dataset. The network "score" is a numerical value used to rank networks according to their degree of relevance to the input dataset. The "score" accounts for the number of experimental focus molecules (proteins) in the network and its size, as well as the total number of proteins in the Ingenuity Knowledge Base that could potentially be included in the specific networks. The network "score" is based on the hypergeometric distribution and is calculated with the right-tailed Fisher's Exact Test. Specific scientific textual associations between filtered protein sets (transmembrane receptor proteins IPA analysis) and Alzheimer's disease processes were created using latent semantic indexing (LSI) algorithms using GeneIndexer (Computable Genomix, Incorporated: https://www.computablegenomix.com/geneindexer). GeneIndexer correlates the strength of association between specific factors (proteins) in a dataset with a user-defined interrogation term. GeneIndexer employs a 2010 murine or human database of over $1 \times 10^{6}$ scientific abstracts to perform text-protein correlation analysis. LSI facilitates the specific textual interrogation of an input dataset with a specific term, that is, Alzheimer's disease, to ascertain which of the input dataset proteins are explicitly associated with the interrogation term. Using LSI algorithms, not only is the direct interrogation term used to analyze the input dataset but also closely correlated additional terms, implicitly associated with the user-defined interrogation term, are also employed in the search patterns. A latent semantic indexing correlation score indicates the strength of association of the interrogation term and the specific proteins in the dataset. A highly relevant protein-term correlation yields a large number of explicitly/implicitly associated proteins with high LSI correlation scores. Therefore, a strong correlation between the proteins in a dataset and a specific user-defined interrogation term yields a large number of correlated proteins with high LSI correlation scores.

\section{Statistical Analysis}

Statistical analysis on multiple samples was performed using a standard nonparametric two-tailed Student's $t$-test using
95\% confidence limits. Analyses were computed using builtin software in GraphPad Prism v. 3.0a (GraphPad Software Inc., La Jolla, CA). Results are expressed as means \pm SE. $P \leq .05$ was considered statistically significant. For statistical analysis using Ingenuity Pathway Analysis v. 8.5 of signaling pathways and interaction network analysis, Fisher's Exact test was employed with a $P \leq .05$ cutoff. Network interaction scores were generated using a right-tailed Fisher's Exact Test.

\section{Results}

4.1. 3xTgAD Mice Demonstrate Impaired Learning and Memory Ability in the Morris Water Maze. Using the nonvisible Morris water maze trial and 16-month-old male control (C57-BL6) and 3xTgAD animals ( $n=10$ for both) we noted that the $3 \times$ TgAD mice demonstrated a significant reduction in their ability to find the location of the hidden platform (Figure 1). The 3xTgAD mice demonstrated significantly longer escape latencies and distances traveled compared to the control mice, while not showing any significant difference in calculated swim speed. Retention testing (three trials one week after the initial training) of these animals (control and 3xTgAD) also demonstrated a reduced cognitive capacity of the $3 \mathrm{xTgAD}$ mice compared to control (data not shown).

4.2. 3xTgAD Mice Demonstrate a Significant Alteration
in Lipid Raft Density and Protein Marker Composition. Employment of the lipid raft isolation process described in the Methods section resulted in the clear visible isolation of a detergent-resistant lipid layer comprising centrifugal fractions 2-4 (Figure 1(a)). The lipid raft marker protein, flotillin-1, was demonstrated to be specifically enriched in these centrifugal fractions (2-4) (Figure 2(a)). The visual lipid density (absorbance units-background/square pixel) of the raft layers was quantified using Fuji-Film Image Gauge. Compared to control, both 8-month-old (Figure 2(c)) and 16-month-old (Figure 2(d)) 3xTgAD-derived centrifugal raft layers demonstrated a significant $(8$ months old $P=$ $.027, n=3 ; 16$ months old $P=.031, n=3)$ increase in buoyant detergent-insoluble density. This 3xTgAD increase in raft size, compared to control animals, demonstrated a strong association with a significant increase in expression of flotillin- 1 in the raft fractions of 3xTgAD mice, especially in centrifugal fraction 2 (Figure $2(\mathrm{e}), P=.017, n=3$ ). Equal levels of total protein (measured using BCA and also SYPRO gel staining) were employed for each Western blot of the raft extracts. Quantification of fraction 2 was chosen, as this reliably indicated the greatest enrichment of this lipid raft marker. Similar quantitative alterations in expression of flotillin- 1 between control and 3xTgAD mice were also seen in the additional lipid raft centrifugal fractions, that is, 3 and 4. Qualitatively similar results, with respect to $3 \mathrm{xTgAD}$ mouse lipid raft density and flotillin-1 expression were noted in parallel experiments carried out with age-matched female mice. In addition we also noted a similar qualitative lipid raft expression of flotillin-1 trend in male human cortex tissue (data not shown). These latter data and their significance 


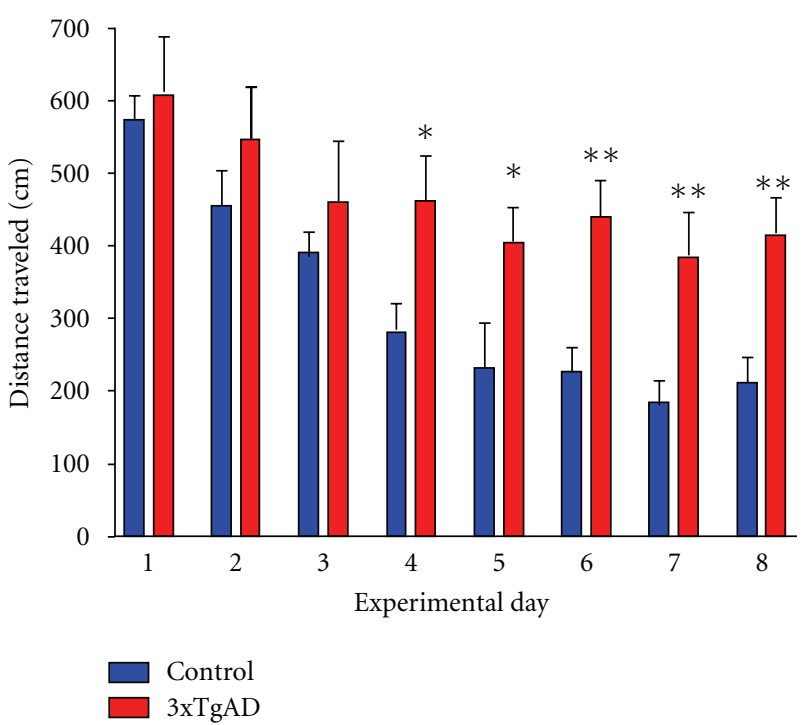

(a)

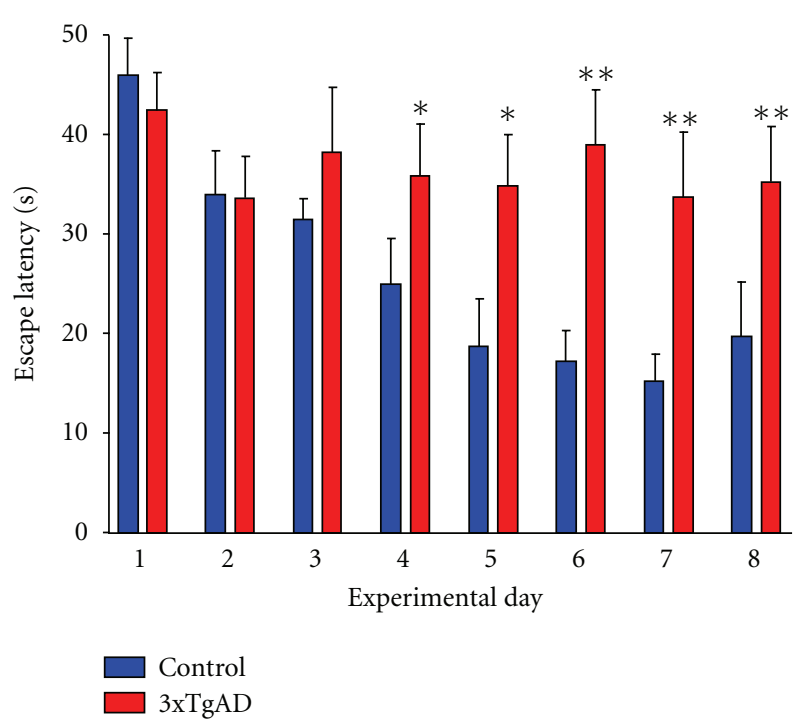

(b)

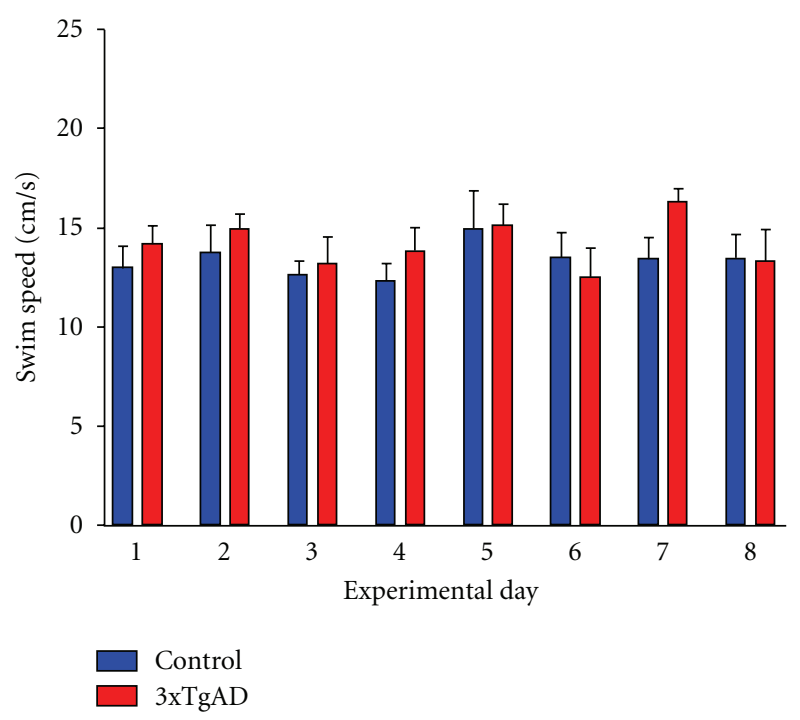

(c)

Figure 1: Morris Water Maze testing of control and 3xTgAD mice. (a) Distance travelled ( $\mathrm{cm}$ ) in the nonvisible probe target results for control ( $n=10$, blue bars) and 3xTgAD mice ( $n=10$, red bars) for 8 days of training. (b) Water maze escape latency (s) for days 1 to 8 of training in the nonvisible probe target. (c) Swim speed $(\mathrm{cm} / \mathrm{s})$ assessment of control and $3 \times \mathrm{xgAD}$ animals during days $1-8$ of training. ${ }^{*} P<.05 ;{ }^{* *} P<.01$.

to our current data will be further addressed in subsequent manuscripts.

\subsection{Differential Protein Expression in Lipid Rafts Isolated} from 3xTgAD Mice Compared to Control Mice. Using an un-biased proteomic analysis of replicate lipid raft extracts, we were able to identify (from at least two individual nonambiguous peptides) multiple proteins in both control and 3xTgAD cortical extracts (control, Appendix A; $3 x \operatorname{TgAD}$, Appendix B). When comparing the relative differences in lipid raft protein expression, only a small minority (17\%: Figure 3(a)) of identified proteins were substantively identified in both control and 3xTgAD raft samples; however many of these common proteins identified were differentially detected (see Supplementary Table 1 in Supplementary Material available online at doi:10.4061/2010/604792). To verify the relative differential expression of multiple proteins in the control versus $3 \times \mathrm{TgAD}$ lipid raft extracts, we also performed multiple Western blot analyses of raft centrifugal fraction-2 (F-2) samples. With loading of total equal protein quantities (50 $\mu \mathrm{g}$ : assessed in an unbiased manner with SYPRO-Ruby: Figure 3(b)) of either control or 3xTgAD F-2 samples, we assessed the relative differential expression of multiple proteins (Figures $3(\mathrm{c})-3(\mathrm{n})$ ). From the Western blot analysis it was consistently demonstrated that 

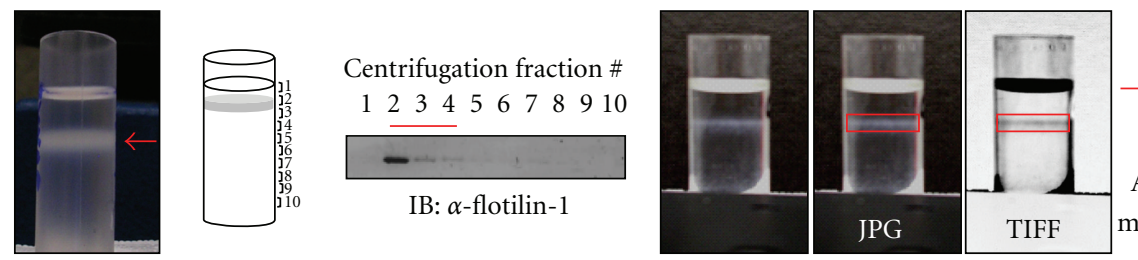

Fuji film-image gauge ${ }^{\mathrm{TM}}(\mathrm{v} 4.2)$

$\rightarrow$ image density processing

(a)

(b)

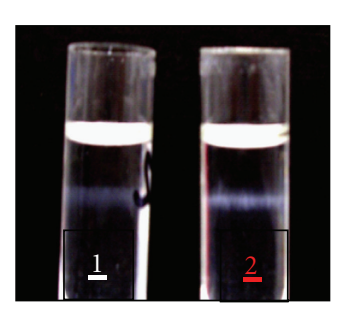

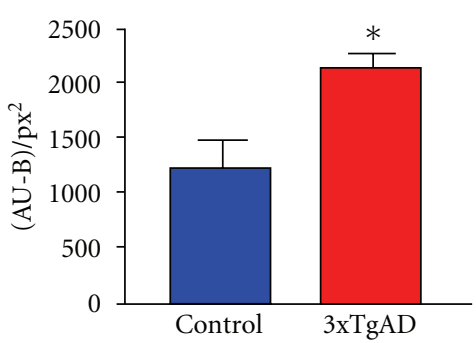

(c)

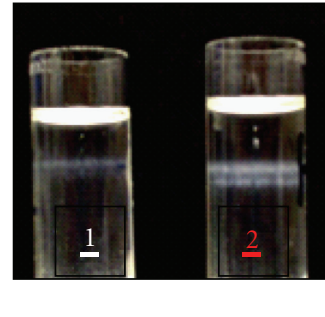

$\begin{array}{lllll}6 & 7 & 8 & 9 & 10\end{array}$

$3 \times \operatorname{TgAD}$

Control

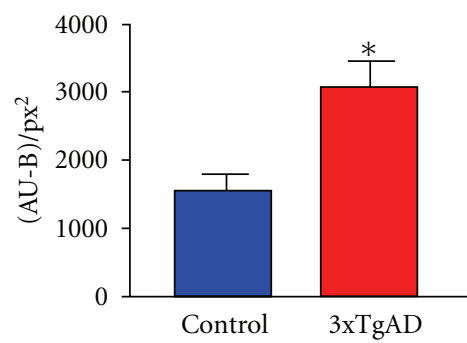

(d)

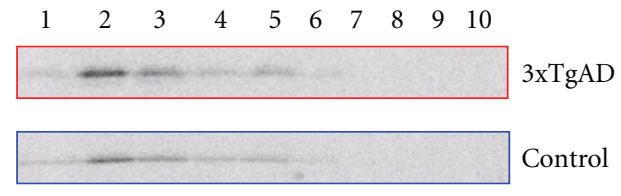

IB: $\alpha$-flotilin-1

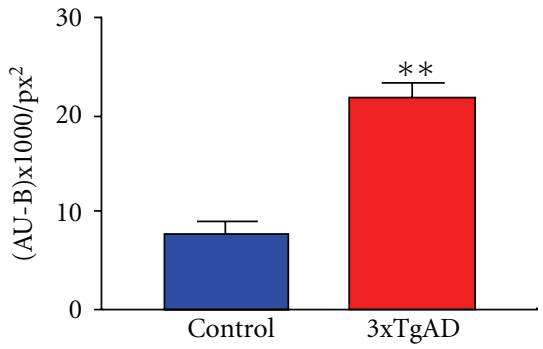

(e)

FIGURE 2: Quantification of detergent-resistant lipid rafts. (a) The pictorial panel depicts an image of iodixanol-separated detergent resistant membrane fractions (centered on red arrow), captured using a Nikon 3200 digital camera. The line diagram indicates the direction of collection of centrifugal fractions 1-10, and the associated Western blot for flotillin-1 demonstrates its enrichment in the raft fractions. (b) Captured Joint Photographic Expert Group (JPG) images were converted to a Tagged Image File Format (TIFF) version and imported to Image Gauge (v4.2) software and the specific area of interest (red box), that is, the detergent-resistant, flotillin-1-rich lipid raft band was quantified into absorbance units minus background absorbance per square pixel area ((AU-B)/px $\left.{ }^{2}\right)$ values. (c) Representative set of male 8-month-old control (1) and 3xTgAD (2) mice detergent-resistant membranes isolated from plasma membrane fractions separated using an Iodixanol gradient. The associated histogram depicts mean \pm s.e. (standard error) mean detergent-resistant membrane intensity $\left((\mathrm{AU}-\mathrm{B}) / \mathrm{px}^{2}\right)$ from at least three separate control and $3 \mathrm{xTgAD}$ mice $(P=.033$, nonpaired, two-tailed $t$-test). (d) Representative set of male 16-month-old control (1) and 3xTgAD (2) mice detergent resistant membranes isolated from plasma membrane fractions separated using an Iodixanol gradient. The associated histogram depicts mean \pm s.e. (standard error) mean detergent resistant membrane intensity ((AU$\left.\mathrm{B}) / \mathrm{px}^{2}\right)$ from at least three separate C57-BL6 and 3xTgAD mice $(P=.0224$, non-paired, two-tailed $t$-test). (e) Representative flotillin-1 Western blot for the lipid raft fraction series for 3xTgAD (red outline) or control (C57-BL6: blue outline) mice. The associated histogram depicts mean \pm s.e. (standard error) mean fraction 2 flotillin expression intensity $\left((\mathrm{AU}-\mathrm{B}) / \mathrm{px}^{2}\right)$ from at least three separate C57-BL6 and $3 x \operatorname{TgAD}$ mice $(P=.015$, non-paired, two-tailed $t$-test).

differential qualitative protein detection in control versus $3 x \operatorname{TgAD}$ raft samples strongly correlated with differential semiquantitative protein expression. Hence, the absence of consistent MS-based detection of Pyk2 (Figure 3(d)), Jak2 (Figure 3(f)), Fyn (Figure 3(h)), paxilin (Figure 3(i)), IRS-1 (Figure 3(j)), caspase 7 (Figure 3(k)), mTOR/FRAP1 (Figure 3(1)) and IGF-1R (Figure 3(n)) in control raft sample correlated to their significantly lower expression in control raft F-2 samples, compared to that in $3 \times \mathrm{TgAD}$ samples. Conversely, the absence of consistent MS-based detection of FAK (Figure 3(c)), GIT-1 (Figure 3(e)), Crk (Figure 3(g)), and Grin2 (Figure $3(\mathrm{~m})$ ), correlated to their significantly lower expression in 3xTgAD raft F-2 samples, compared to that in control samples. 


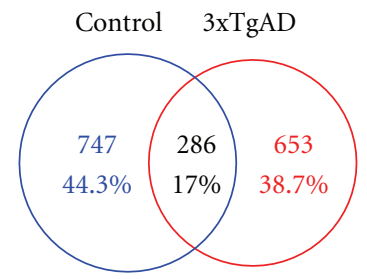

(a)

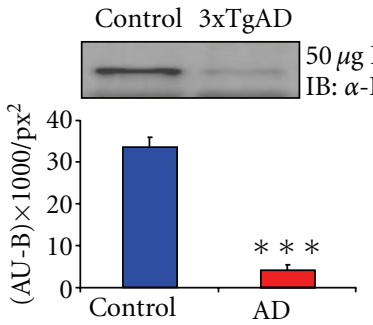

(c)

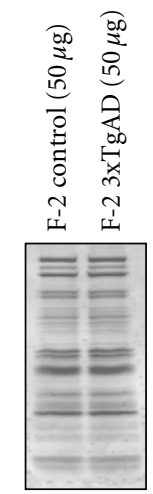

SYPRO Ruby

(b)

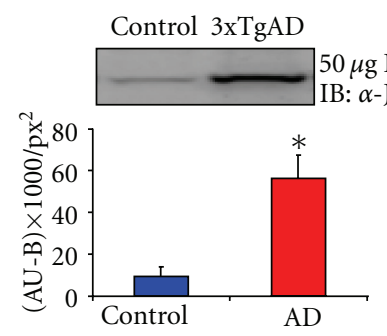

(f)

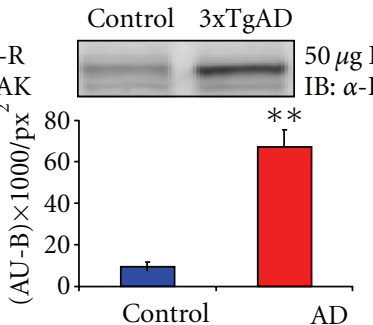

(d)

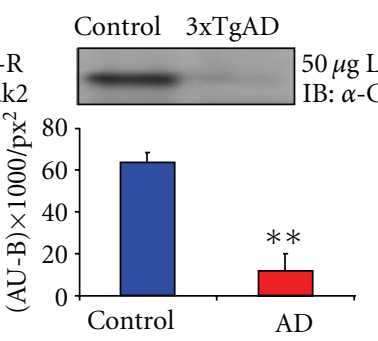

(g)

Control 3xTgAD

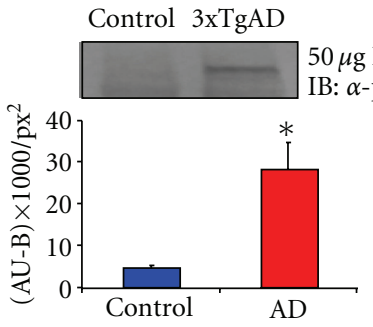

(i)

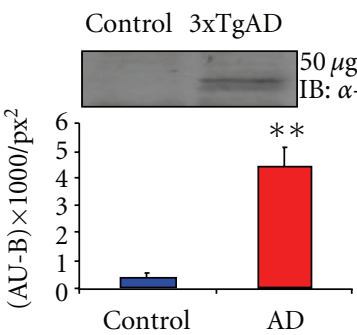

(1)

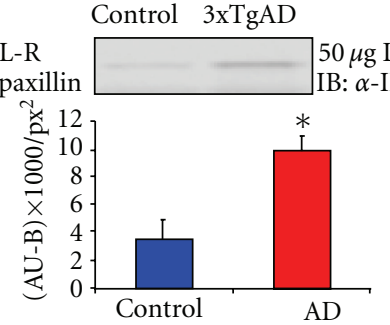

(j)

Control 3xTgAD

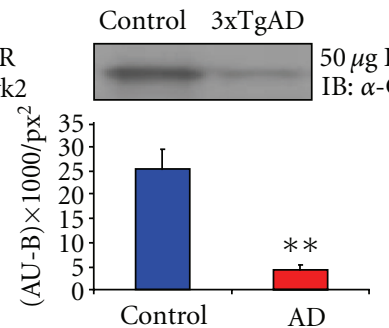

(e)

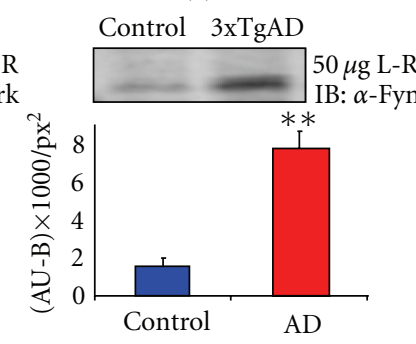

(h)
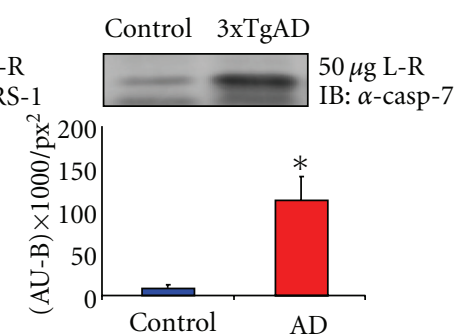

(k)

Control 3xTgAD

(n)

Figure 3: Differential protein expression in control versus 3xTgAD lipid raft extracts. (a) Proportionately drawn Venn diagram analysis of reliably identified proteins from control lipid rafts (blue line) and 3xTgAD rafts (red line). (b) Total protein loading control for centrifugal fraction 2. A total of $50 \mathrm{mg}$ of fraction 2 protein was loaded and stained with SYPRO Ruby and scanned using a phosphorimager. (c)-(n). Representative western blots from multiple expression analysis experiments for differential presentation of proteins in fraction 2 extracts from control (blue) or 3xTgAD mice (red). Associated with each panel (c)-(n) the associated histograms represent the mean \pm s.e. mean of protein expression intensity (measured in $\left((\mathrm{AU}-\mathrm{B}) / \mathrm{px}_{2}\right)$ ) from at least three separate experiments. ${ }^{*} P<.05 ;{ }^{* *} P<.01 ;{ }^{* * *} P<.001$. Protein abbreviations are as follows. FAK: focal adhesion kinase; Pyk2: proline-rich tyrosine kinase 2; GIT-1: GRK interactor-1; Jak2: Janus kinase 2; Crk: v-Crk avian sarcoma virus CT10 oncogene homolog; Fyn: Fyn tyrosine kinase; IRS-1: insulin receptor substrate-1; casp 7: caspase 7; mTOR: mammalian target of rapamycin; Grin2: G protein-regulated inducer of neurite outgrowth 2; IGF-1R: insulin-like growth factor-1 receptor. 


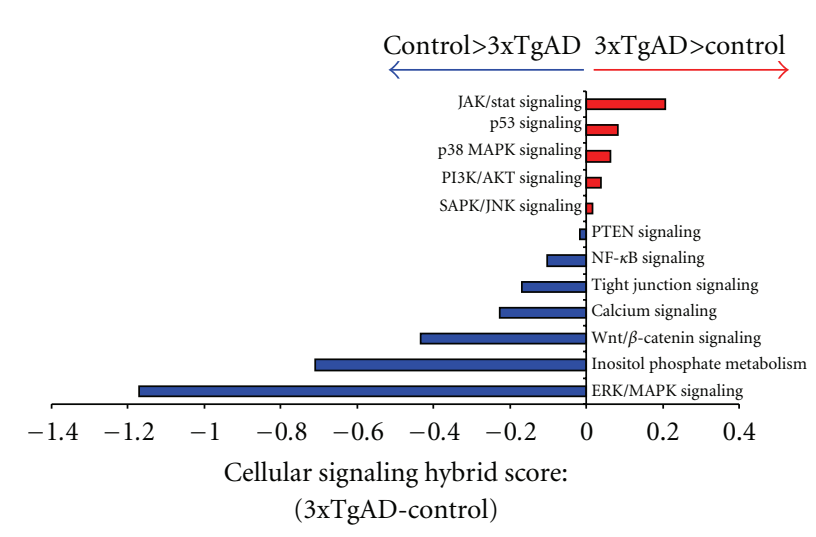

(a)

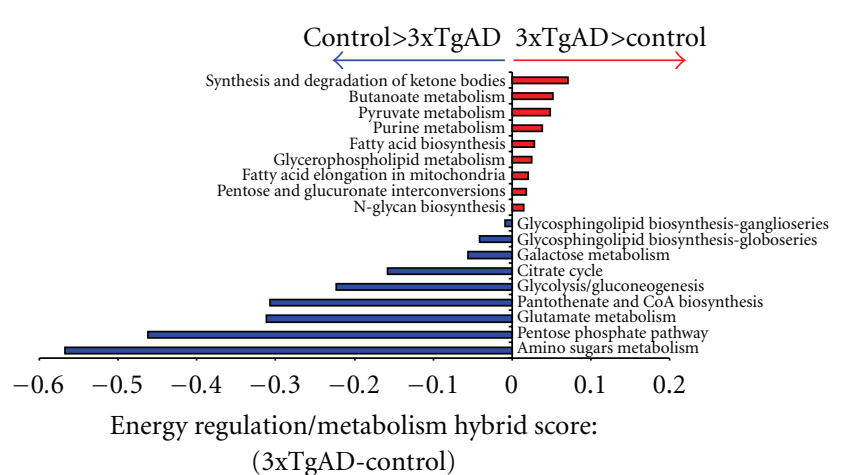

(c)

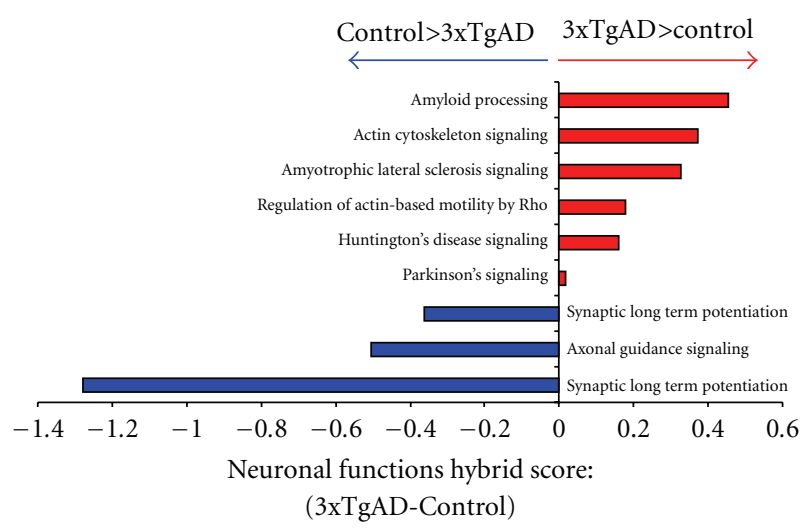

(b)

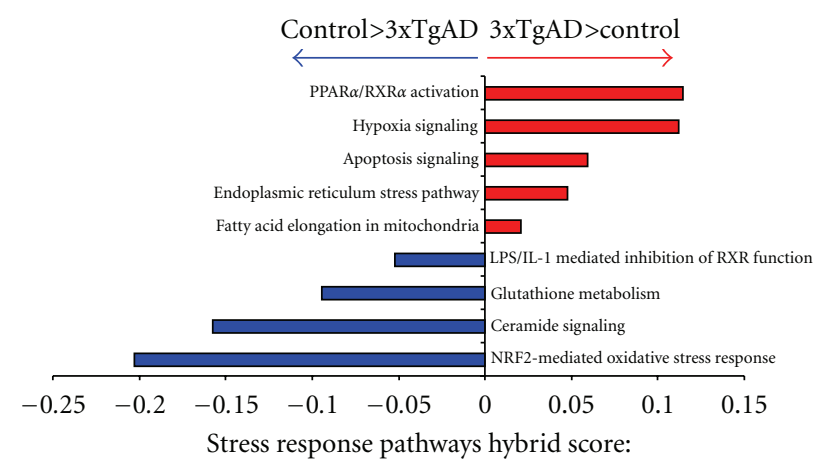

(3xTgAD-control)

(d)

FIGURE 4: Functional pathway informatic clustering of control and 3xTgAD lipid raft proteins. (a) Subtractive representation of hybrid score generation after clustering of lipid raft proteins from control and 3xTgAD animal rafts into cellular signaling pathways. The hybrid scores were generated by multiplication of the protein enrichment ratio of the specific pathway with the negative $\log _{10}\left(-\log _{10}\right)$ of the probability of that enrichment (see Section 2). The data is presented as a numerical value of the control pathway hybrid score subtracted from the 3xTgAD pathway hybrid score. Pathways in which the score in $3 \mathrm{xTgAD}$ was greater than the control are denoted in red; pathways in which the control hybrid score is greater than the $3 x \mathrm{TgAD}$ hybrid score are denoted in blue. A similar depiction format is employed for differential analysis of control versus 3xTgAD Neuronal Function pathways (b), Energy Regulation/Metabolism pathways (c), and Stress Response pathways (d).

4.4. Functional Signaling Cluster Analysis of Control versus 3xTgAD Lipid Raft Proteomes. As our MS-based multidimensional protein identification process identified several hundred proteins from each control or 3xTgAD lipid raft sample, we employed a bioinformatic analysis process to assess the relative functionalities of both the control versus $3 \mathrm{xTgAD}$ raft protein lists. As the majority of cellular signaling processes are mediated and regulated by multiple groups of proteins interacting with each other, we clustered, in a statistically significant manner, proteins in control or $3 x T g A D$ animal raft samples into functional signaling groups. To assess the relative changes in regulation of classical signaling pathways, we applied a subtractive approach for the pathway "hybrid" scores (indicative of the "activity" of the specific signaling pathway: calculated by significant expression enrichment ratio of proteins in that pathway multiplied by the negative $\log _{10}\left(-\log _{10}\right)$ of the probability of the pathway enrichment). For each specific common signaling pathway, our mathematical approach subtracted control pathway "hybrid" scores from the pathway "hybrid" scores generated from the $3 x \operatorname{TgAD}$ protein set. Hence, a positive result of this subtraction indicates a greater activity of this functional pathway in 3xTgAD animals, and a negative score indicates a greater activity of this functional pathway in the control animals. Analysis of pathways involved in cellular signaling (Figure 4(a): proteins and scores in associated Appendix C) demonstrated that pathways commonly associated with cell stress responses were highly activated in 3xTgAD rafts, for example "p53 signaling", "p38 mitogenactivated protein kinase (MAPK) signaling", and "stressactivated protein kinase (SAPK)/JNK pathways". In a stark contrast, prosurvival synaptic connectivity and neurotransmissive pathways were more profoundly activated in the control mice, for example, "tight junction signaling”, "calcium signaling", "PTEN signaling", and "Wnt/ $\beta$-catenin signaling". To investigate the specific neuronal functional effects of these disparate signaling activities, we next studied the significant clustering of raft proteins into neuron-functional pathways (Figure 4(b): Appendix D). As one would expect, the $3 x \mathrm{TgAD}$ mice raft protein clustering revealed a considerably 
greater (relative to control) activation of multiple neurodegenerative neuronal processes including: "amyloid processing", "Amyotrophic lateral sclerosis", "Huntington's disease signaling" and "Parkinson's signaling". In addition to the greater activation of these degenerative processes, the 3xTgAD mice also demonstrated profound changes in the significant clustering of proteins into cytoskeletal remodeling groups ("actin cytoskeleton signaling", regulation of actin-based motility by Rho') compared to the control mice. In accordance with our demonstration of the significant diminution of the learning and memory ability of the $3 \times \mathrm{TgAD}$ mice, it was striking to notice the profoundly greater activation of neuron-functional pathways that control synaptic learningdependent processes (i.e., "synaptic long-term depression", "axonal guidance signaling", and "synaptic long-term potentiation") in the control mice compared to the $3 \mathrm{xTgAD}$ mice. Considerable evidence from multiple experimental studies has recently underlined the importance of the regulation of energy metabolism in controlling the aging process and neurodegenerative disorders [28-30]. Upon inspection of the relative differences in the activation of energy-regulatory pathways created by clustering of control raft proteins versus $3 \times \operatorname{TgAD}$ raft proteins, a profound functional distinction was noted (Figure 4(c): Appendix E). In control animals versus $3 \mathrm{xTgAD}$, there was a considerably stronger activation of energy-generating pathways connected to the use of the primary metabolic substrate, that is, sugars ("amino sugars metabolism", "glycolysis/gluconeogenesis", and "pentose phosphate pathway"). In contrast, the energy regulatory pathways that were more strongly associated with the $3 x \operatorname{TgAD}$ animals involved energy derivation from alternative energy sources, for example, "synthesis and degradation of ketone bodies", "butanoate metabolism", and "fatty acid biosynthesis". Many of the alterations in energy regulation in aging and degenerative disorders are thought to be associated with adaptive responses to the induction of cellular stresses, potentially through toxic effects of $A \beta$, NFTs and accumulated oxidative damage [30]. When the raft proteins from control and $3 \times \mathrm{TgAD}$ mice were clustered into functional stress response pathways, again a stark contrast in the control- or 3xTgAD-associated pathways was demonstrated (Figure 4(d): Appendix F). In the 3xTgAD mice raft clustering it was noted that the association of energyassociated stressful and neuronal damage-related pathways ("PPAR $\alpha / R X R \alpha$ activation", "hypoxia signaling", "apoptosis signaling", and "endoplasmic reticulum stress pathway") was considerably stronger than in the control mice. Indicating a correlated connection between stress response capacity and $\mathrm{AD}$ pathology, there was a considerably greater association of the "Nrf2-mediated oxidative stress response pathway" in control mice compared to the $3 \mathrm{xTgAD}$. Therefore, the $3 x \operatorname{TgAD}$ mice may demonstrate excessive neuronal stress and damage due to the attenuated activation of such stress response pathways in lipid rafts of $\mathrm{AD}$ synapses.

4.5. Functional Receptor Signaling Cluster Analysis of Control versus $3 \times T g A D$ Lipid Raft Proteomes. As one of the most important functions of synaptic lipid rafts is to congregate transmembrane or juxtamembrane receptor systems [31], we next performed an in-depth investigation of the significant differential functional clustering of receptor signaling pathways between control and 3xTgAD rafts. Upon functional clustering of the raft proteins into receptor signaling pathways, strong differences in pathways association between control and 3xTgAD mice were noted (Figure 5: Appendix G). Some of the strongest differences were noted by the considerably poorer activation of growth factor-related signaling ("PDGF signaling", "EGF signaling", and "FGF signaling"), structural trans-synaptic receptor signaling ("Neuregulin signaling" and "Ephrin receptor signaling"), excitatory signaling (glutamate receptor signaling) and neurodevelopmental signaling (Sonic hedgehog signaling) pathways in the $3 \mathrm{xTgAD}$ mice, compared to the control mice. The receptor signaling profile of the $3 \times \mathrm{TgAD}$ mice demonstrated a more profound association compared to control mice for pathways linked to inhibitory synaptic signaling ("GABA receptor signaling" and "Aryl hydrocarbon receptor signaling": [32]), amyloid processing ("Notch signaling" and "Integrin signaling": [33]), and neuronal stress (glucocorticoid receptor signaling). Transmembrane receptor signaling by systems including receptor tyrosine kinases or G protein-coupled receptors (GPCRs) represents one of the most important signaling mechanisms of neuronal synaptic regulation [34]. However, the activation of transmembrane receptor systems and the stimulation of their intracellular signaling cascades, especially for GPCRs, is now considered to be far more complex and intricate than initially proposed by two-state receptor models [34, 35]. Much of this additional signaling diversity is thought to arise from the additional complexity of receptor-accessory scaffolding protein modification of receptor signaling [35]. To appreciate the multiple connections between receptor (and GPCR in particular) activity and the presence of neurophysiological deficits in $\mathrm{AD}$, we performed a multidimensional analysis of the proteins present in control or $3 \mathrm{xTgAD}$ mice. Using the novel, un-biased, bioinformatic GeneIndexer latent semantic indexing (LSI) process (https://www.computablegenomix.com/geneindexer.php), explicit correlations can be made between protein/gene factors from input datasets and their linkage (in over $1 \times 10^{6}$ curated scientific abstracts) to a specific interrogation term, for example, Alzheimer's. The semantic indexing algorithms of GeneIndexer also allow for multidimensional correlations to be measured for terms significantly related to the interrogation term, hence providing a flexible, intelligent query process. For the control and 3xTgAD datasets, we employed multiple interrogation terms targeted to demonstrate differences between control and $3 \times \mathrm{TgAD}$ datasets. Using the following interrogation terms: Alzheimer's, oxidation, neurodegeneration, synaptic transmission, neurogenesis, scaffolding, and GPCR, we demonstrated that, at a multidimensional interactive level, there is minimal functional cross-over between control and 3xTgAD raft samples (Figure 6: Appendix H). Only proteins that demonstrated explicit correlations (latent semantic indexing score of $\geq 0.1$ ) to at least two of the interrogation 


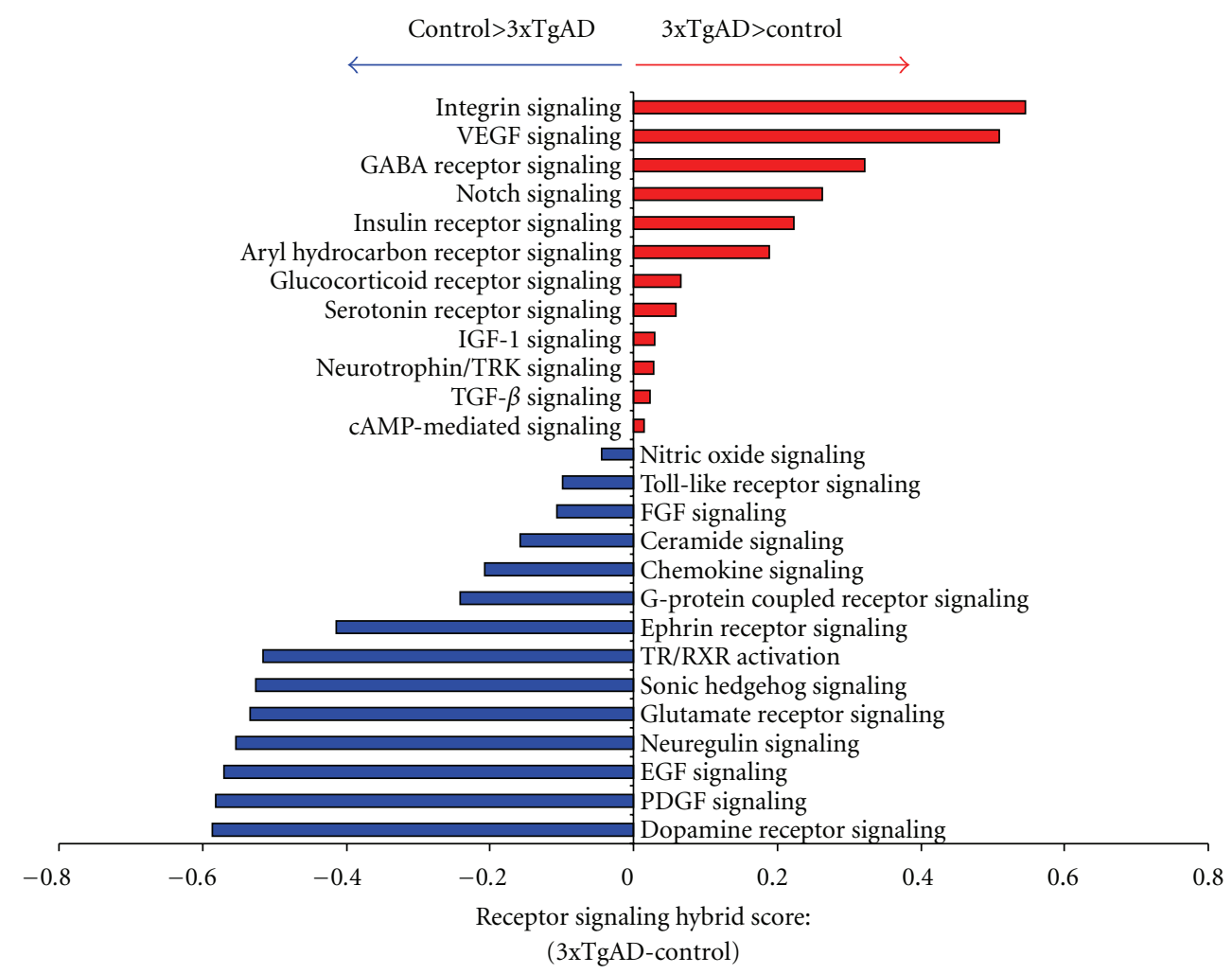

FIGURE 5: Functional receptor signaling pathway informatic clustering of control and 3xTgAD lipid raft proteins. Subtractive representation of hybrid score generation after clustering of raft proteins from control and 3xTgAD animal rafts into receptor signaling pathways. The data is presented as a numerical value of the control pathway hybrid score subtracted from the 3xTgAD pathway hybrid score. Pathways in which the score in 3xTgAD was greater than the control are denoted in red; pathways in which the control hybrid score was greater than the $3 \mathrm{xTgAD}$ hybrid score are denoted in blue.

terms are denoted in the multidimensional heatmap (Figure 6: Appendix H). Therefore, each of the proteins identified in the heatmap are strongly correlated with many of the connected interrogation terms and therefore show potential synergistic activity. Analysis of identified proteins in this manner, that is, selected specifically for multidimensional neurological roles, allows for an unbiased focusing on proteins that may possess keystone-like functions in the molecular signaling networks involved in neurodegeneration. With respect to specific interrogation terms, a strong validation of the technique is demonstrated by the fact that using the Alzheimer's interrogation term, 47 3xTgAD-unique multidimensional proteins were indicated while only 10 such multidimensional proteins were shown in control rafts (Figure 6). Confirming a strong role of oxidative damage, $213 x \operatorname{TgAD}$-unique multidimensional proteins were present in the oxidation results while only 6 such control-unique were demonstrated. Interestingly and perhaps suggestive of a potential future line of $\mathrm{AD}$ research was the observation that considerably fewer multidimensional 3xTgAD-unique proteins were associated with the process of neurogenesis (15), compared to the 32 control-unique multidimensional proteins associated with this neuroprotective mechanism. In accordance with the important role of receptor systems in $\mathrm{AD}$, we additionally noted that more $3 \mathrm{xTgAD}$-unique proteins were associated with GPCRs (27) compared to control-unique GPCR-related multidimensional proteins (16).

\subsection{Functional Interaction Networks of Receptor Signaling} Proteins in Control versus 3xTgAD Lipid Raft Proteomes. From our investigation of the multidimensional nature of raft proteins with respect to neurodegenerative processes, it is clear that there are many factors that are highly likely to work together in complex and intricate functional networks. To investigate the nature of the most statistically likely functional network interactions, we employed IPA network analysis of a receptor-filtered (using IPA-knowledge base data filtering, IPA v. 8,5) subset of the control or $3 x$ TgAD raft protein datasets (Tables 1 and 2, resp.). Using these datasets, un-biased network analysis is able to predict the most likely series of functional interactions (based on empirically derived experimental evidence) that take place between the receptor-associated raft proteins. The control and $3 \times$ TgAD receptor-specific filtered datasets demonstrated a relatively minimal overlap, that is, $11.6 \%$ commonality, indicating that substantial alterations of these proteins may occur in the rafts of 3xTgAD animals (Figure 7(a)). The most statistically likely interaction network that was predicted to occur in control mice centered on neuroprotective and neurotransmissive factors such as phosphoinositide-3-kinse 

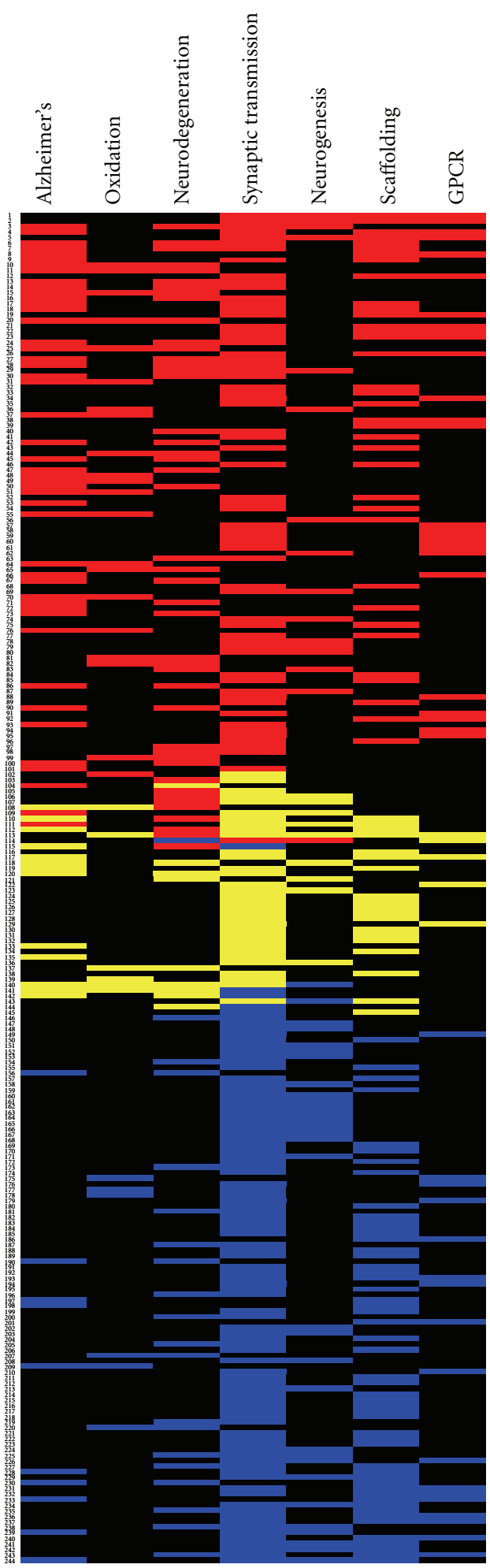

$3 \times \operatorname{TgAD}$

$3 \mathrm{xTgAD}+$ control

Control

FIgURE 6: Multidimensional protein latent semantic indexing (LSI) analysis of proteins extracted from control and 3xTgAD lipid rafts. Proteins from the control or 3xTgAD extracted datasets that possessed an explicit latent semantic indexing (LSI, GeneIndexer, Computable Genomix) score in at least two of the multiple GeneIndexer interrogation terms (Alzheimer's, oxidation, neurodegeneration, synaptic transmission, neurogenesis, scaffolding, and GPCR) are represented in a heatmap format. Proteins are identified on the left side of the heatmap as an individual number (see Appendix H for key). The presence of a colored panel (3xTgAD, red: control, blue: 3xTgAD and control: yellow) on the same lateral as the numbered protein denotes explicit textual correlation of that protein with the specific vertical interrogation term. 
TABLE 1: Receptor-specific protein list from lipid raft extracts from control animals. Primary protein lists of extracted lipid raft proteins were filtered for a specific receptor profile using IPA v. 8.5.

\begin{tabular}{|c|c|c|c|}
\hline Symbol & Protein definition & Location & Protein type \\
\hline $\mathrm{Cd} 4$ & CD4 molecule & Plasma membrane & Transmembrane receptor \\
\hline Celsr2 & Cadherin, EGF LAG seven-pass G-type receptor 2 & Plasma membrane & G-protein coupled receptor \\
\hline Celsr3 & Cadherin, EGF LAG seven-pass G-type receptor 3 & Plasma membrane & G-protein coupled receptor \\
\hline Chrm5 & Cholinergic receptor, muscarinic 5 & Plasma membrane & G-protein coupled receptor \\
\hline Chrnb3 & Cholinergic receptor, nicotinic, beta 3 & Plasma membrane & Transmembrane receptor \\
\hline Chrnb4 & Cholinergic receptor, nicotinic, beta 4 & Plasma membrane & Transmembrane receptor \\
\hline Chrng & Cholinergic receptor, nicotinic, gamma & Plasma membrane & Transmembrane receptor \\
\hline Cldn4 & Claudin 4 & Plasma membrane & Transmembrane receptor \\
\hline Clecsf6 & C-type lectin domain family 4 , member A & Plasma membrane & Transmembrane receptor \\
\hline Cnr1 & Cannabinoid receptor 1 (brain) & Plasma membrane & G-protein coupled receptor \\
\hline Cntfr & Ciliary neurotrophic factor receptor & Plasma membrane & Transmembrane receptor \\
\hline Cxadr & Coxsackie virus and adenovirus receptor & Plasma membrane & Transmembrane receptor \\
\hline Dag1 & Dystroglycan 1 (dystrophin-associated glycoprotein 1) & Plasma membrane & Transmembrane receptor \\
\hline Ednrb & Endothelin receptor type B & Plasma membrane & G-protein coupled receptor \\
\hline Fshr & Follicle stimulating hormone receptor & Plasma membrane & G-protein coupled receptor \\
\hline Fzd9 & Frizzled homolog 9 (Drosophila) & Plasma membrane & G-protein coupled receptor \\
\hline Gabbr1 & Gamma-aminobutyric acid (GABA) B receptor, 1 & Plasma membrane & G-protein coupled receptor \\
\hline Gabrr3 & Gamma-aminobutyric acid (GABA) receptor, rho 3 & Plasma membrane & Transmembrane receptor \\
\hline Gcgr & Glucagon receptor & Plasma membrane & G-protein coupled receptor \\
\hline Gfral & GDNF family receptor alpha 1 & Plasma membrane & Transmembrane receptor \\
\hline Gfra3 & GDNF family receptor alpha 3 & Plasma membrane & Transmembrane receptor \\
\hline Gpr116 & G protein-coupled receptor 116 & Plasma membrane & G-protein coupled receptor \\
\hline Gpr141 & G protein-coupled receptor 141 & Plasma membrane & G-protein coupled receptor \\
\hline Gprc6a & G protein-coupled receptor, family $\mathrm{C}$, group 6 , member A & Plasma membrane & G-protein coupled receptor \\
\hline Grm4 & Glutamate receptor, metabotropic 4 & Plasma membrane & G-protein coupled receptor \\
\hline Grm5 & Glutamate receptor, metabotropic 5 & Plasma membrane & G-protein coupled receptor \\
\hline Cbp & Opsin 1 (cone pigments), long-wave-sensitive & Plasma membrane & G-protein coupled receptor \\
\hline Oprd1 & Opioid receptor, delta 1 & Plasma membrane & G-protein coupled receptor \\
\hline Osmr & Oncostatin $\mathrm{M}$ receptor & Plasma membrane & Transmembrane receptor \\
\hline Prom2 & Prominin 2 & Plasma membrane & Transmembrane receptor \\
\hline Prph2 & Peripherin 2 (retinal degeneration, slow) & Plasma membrane & Transmembrane receptor \\
\hline Ptger2 & Prostaglandin E receptor 2 (subtype EP2), 53kda & Plasma membrane & G-protein coupled receptor \\
\hline Pthr2 & Parathyroid hormone 2 receptor & Plasma membrane & G-protein coupled receptor \\
\hline Sfrp4 & Secreted frizzled-related protein 4 & Plasma membrane & Transmembrane receptor \\
\hline Smo & Smoothened homolog (Drosophila) & Plasma membrane & G-protein coupled receptor \\
\hline Sstr2 & Somatostatin receptor 2 & Plasma membrane & G-protein coupled receptor \\
\hline Tacr1 & Tachykinin receptor 1 & Plasma membrane & G-protein coupled receptor \\
\hline Tas2r41 & Taste receptor, type 2 , member 41 & Plasma membrane & G-protein coupled receptor \\
\hline Thbd & Thrombomodulin & Plasma membrane & Transmembrane receptor \\
\hline Tlr3 & Toll-like receptor 3 & Plasma membrane & Transmembrane receptor \\
\hline Tlr4 & Toll-like receptor 4 & Plasma membrane & Transmembrane receptor \\
\hline Tlr5 & Toll-like receptor 5 & Plasma membrane & Transmembrane receptor \\
\hline Tlr6 & Toll-like receptor 6 & Plasma membrane & Transmembrane receptor \\
\hline Tlr9 & Toll-like receptor 9 & Plasma membrane & Transmembrane receptor \\
\hline Tshr & Thyroid stimulating hormone receptor & Plasma membrane & G-protein coupled receptor \\
\hline Unc5b & Unc-5 homolog B (C. Elegans) & Plasma membrane & Transmembrane receptor \\
\hline Vipr2 & Vasoactive intestinal peptide receptor 2 & Plasma membrane & G-protein coupled receptor \\
\hline
\end{tabular}


TABLE 2: Receptor-specific protein list from lipid raft extracts from $3 x T g A D$ animals. Primary protein lists of extracted lipid raft proteins were filtered for a specific receptor profile using IPA v. 8.5.

\begin{tabular}{|c|c|c|c|}
\hline Symbol & Protein definition & Location & Protein type \\
\hline Adcyap1r1 & Adenylate cyclase activating polypeptide 1 (pituitary) receptor type I & Plasma membrane & G-protein coupled receptor \\
\hline $\mathrm{Cd} 36$ & CD36 molecule (thrombospondin receptor) & Plasma membrane & Transmembrane receptor \\
\hline Cd86 & CD86 molecule & Plasma membrane & Transmembrane receptor \\
\hline Cnr1 & Cannabinoid receptor 1 (brain) & Plasma membrane & G-protein coupled receptor \\
\hline Cxadr & Coxsackie virus and adenovirus receptor & Plasma membrane & Transmembrane receptor \\
\hline Gabbr1 & Gamma-aminobutyric acid (GABA) B receptor, 1 & Plasma membrane & G-protein coupled receptor \\
\hline Gpr1 & G protein-coupled receptor 1 & Plasma membrane & G-protein coupled receptor \\
\hline Gpr141 & G protein-coupled receptor 141 & Plasma membrane & G-protein coupled receptor \\
\hline Gpr56 & G protein-coupled receptor 56 & Plasma membrane & G-protein coupled receptor \\
\hline Htr2c & 5-hydroxytryptamine (serotonin) receptor $2 \mathrm{C}$ & Plasma membrane & G-protein coupled receptor \\
\hline Htr4 & 5-hydroxytryptamine (serotonin) receptor 4 & Plasma membrane & G-protein coupled receptor \\
\hline Igf1r & Insulin-like growth factor 1 receptor & Plasma membrane & Transmembrane receptor \\
\hline Igf2r & Insulin-like growth factor 2 receptor & Plasma membrane & Transmembrane receptor \\
\hline Il1r2 & Interleukin 1 receptor, type II & Plasma membrane & Transmembrane receptor \\
\hline Il1rapl1 & Interleukin 1 receptor accessory protein-like 1 & Plasma membrane & Transmembrane receptor \\
\hline Lifr & Leukemia inhibitory factor receptor alpha & Plasma membrane & Transmembrane receptor \\
\hline Lphn 1 & Latrophilin 1 & Plasma membrane & G-protein coupled receptor \\
\hline Lphn2 & Latrophilin 2 & Plasma membrane & G-protein coupled receptor \\
\hline Lrpap 1 & Low density lipoprotein receptor-related protein associated protein 1 & Plasma membrane & Transmembrane receptor \\
\hline Npr2 & Natriuretic peptide receptor B/guanylate cyclase B & Plasma membrane & G-protein coupled receptor \\
\hline Npy5r & Neuropeptide Y receptor Y5 & Plasma membrane & G-protein coupled receptor \\
\hline Omg & Oligodendrocyte myelin glycoprotein & Plasma membrane & G-protein coupled receptor \\
\hline Cbp & Opsin 1 (cone pigments), long-wave-sensitive & Plasma membrane & G-protein coupled receptor \\
\hline Ptch1 & Patched homolog 1 (Drosophila) & Plasma membrane & Transmembrane receptor \\
\hline Pthr2 & Parathyroid hormone 2 receptor & Plasma membrane & G-protein coupled receptor \\
\hline Robol & Roundabout, axon guidance receptor, homolog 1 (Drosophila) & Plasma membrane & Transmembrane receptor \\
\hline Lgr7 & Relaxin/insulin-like family peptide receptor 1 & Plasma membrane & G-protein coupled receptor \\
\hline Tlr5 & Toll-like receptor 5 & Plasma membrane & Transmembrane receptor \\
\hline Tnfrsf14 & Tumor necrosis factor receptor superfamily, member 14 & Plasma membrane & Transmembrane receptor \\
\hline Tshr & Thyroid stimulating hormone receptor & Plasma membrane & G-protein coupled receptor \\
\hline
\end{tabular}

(PI3K), Akt-1, muscarinic GPCRs (Chrm5), and glutamate receptors (Grm5) (Figure 7(a): Appendix I). In contrast, the highest statistically scoring network from $3 \times \mathrm{TgAD}$ receptorspecific proteins was centered on lipid-regulating factors and stress-related factors including: p38 MAPK; Jnk (cjun $\mathrm{N}$-terminal kinase), Lrpap1 (low-density lipoprotein receptor-related protein associated protein 1); LDL (low density lipoprotein), and NF- $\kappa$ B (Figure 7(b): Appendix J). Therefore, at the level of functional interaction of receptorrelated proteins in the lipid rafts, it is clear that these membrane microdomains are a strong functional locus of this degenerative disease. Reinforcing this AD-relevant microcosm effect in the lipid rafts we analyzed, using latent semantic indexing (LSI) interrogation of these receptorspecific datasets (Table 2-control; Appendix I-3xTgAD), the Alzheimer's disease correlation of these receptor-specific proteins. In Figure 8, we demonstrate that almost twice as many proteins in the $3 \mathrm{xTgAD}$ dataset (21) explicitly correlated with the interrogation term Alzheimer's disease compared to the proteins in the control dataset (11). The degree of correlation of the interrogation term (Alzheimer's) to each specific protein is indicated by the LSI score. In Figure 8(b) the cumulated LSI score for the 3xTgAD Alzheimer's-related proteins was 2.92 while for the control proteins only 1.21 (Figure $8(\mathrm{~b})$ ), demonstrating that a much stronger correlation of receptor-associated proteins existed for the proteins in $3 \mathrm{xTgAD}$ rafts, compared to control. Upon comparison of the phylogenetic relationships of the receptor-specific proteins that form the most likely functional networks (from Figure 7(b)), it is clear that for the control-set proteins only three of these in this network are highly correlated to reports of Alzheimer's disease (27.2\%: Figure 8(c)) while the most coherent interaction network of $3 \mathrm{xTgAD}$ raft proteins possessed a much higher percentage of Alzheimer's disease-related proteins (62\%: Figure 8(d)). Therefore, this suggests that one of the important pathological loci of $\mathrm{AD}$ could be the disruption of interactivity of receptor-associated proteins in the lipid raft microdomains 


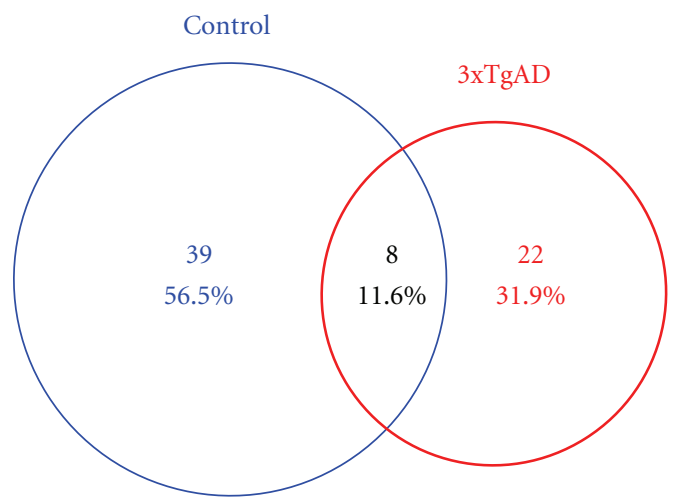

(a)

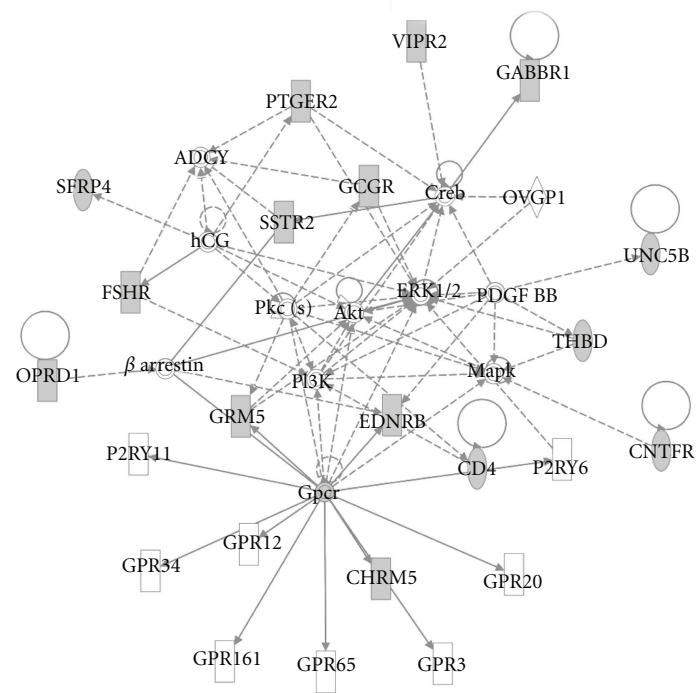

(b)

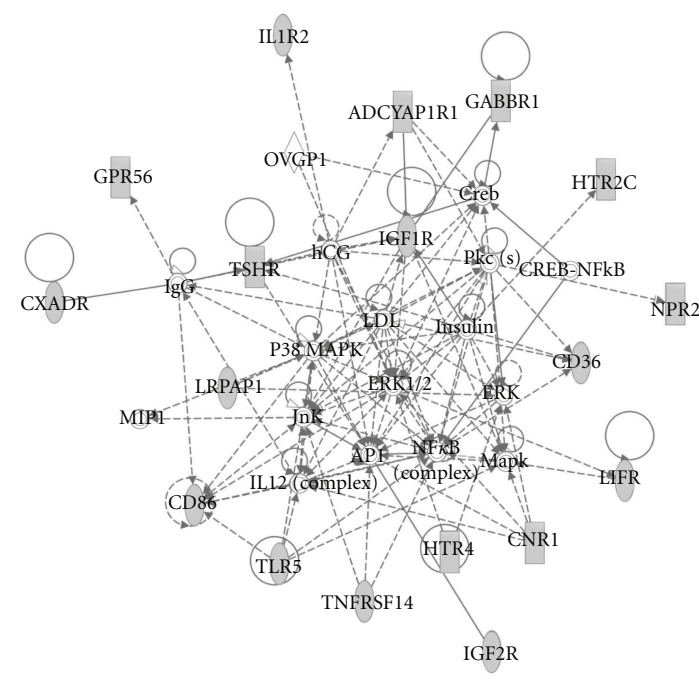

(c)

Figure 7: Receptor-restricted control and 3xTgAD network interaction analysis. (a) Proportionally drawn Venn diagram depicting the relative distribution between control or 3xTgAD raft samples of receptor-specific proteins filtered using IPA version 8.5 (control filtered protein list, Table 1; 3xTgAD filtered protein list, Table 2). (b) The highest scoring protein interaction network generated from IPA Network analysis (network scores and focus molecules are listed in Appendix I) of the receptor-specific control dataset. (c) The highest scoring protein interaction network generated from IPA Network analysis (network scores and focus molecules are listed in Appendix I) of the receptor-specific 3xTgAD dataset (Appendix J). A full description of the nature of interactions based on the connecting lines can be found at the following webpage linked to the IPA analysis module (https://analysis.ingenuity.com/pa/info/help/help.htm\#ipa_help.htm). Dashed lines represent indirect gene interactions while solid lines represent empirically measured direct interactions. The two highest significantly scoring networks (B-control, C-3xTgAD) are based on the highest percentage of the network occupation by specific proteins (focus molecules) from the input receptor-specific datasets.

and that such a finding can be uncovered in a completely unbiased informatic format from extremely large and difficult to interpret datasets.

\section{Discussion}

Multiple informatic techniques were employed to investigate and elucidate the nature of functional protein interactions that occur differentially in the lipid raft microdomains of control versus $3 \times \mathrm{TgAD}$ Alzheimer's disease mice. Cognitively impaired male 3xTgAD mice showed profound differences in the protein constituents of lipid raft extracts compared to age-matched control mice, that is, only $17 \%$ of raft proteins were similar between the two groups of mice. Our microdomain proteomic approach was able to specifically assist in the identification of altered proteins that are highly characteristic of AD-related raft pathophysiology, for example, the Src-family tyrosine kinase Fyn (Figure 3(h)) $[19,20]$.

Currently, biological scientists are often faced with complex biological issues concerning the interpretation of their results due to the development of facile mass data acquisition technologies, that is, extracting relevant and illuminating information from large datasets is often extremely challenging. However, with the application of multiple, sequential 


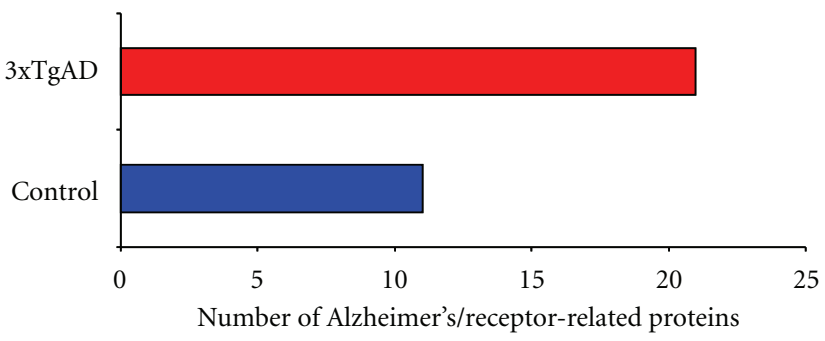

(a)

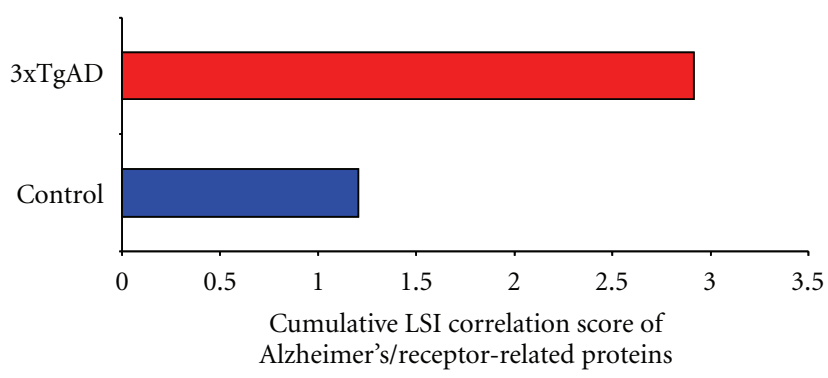

(b)

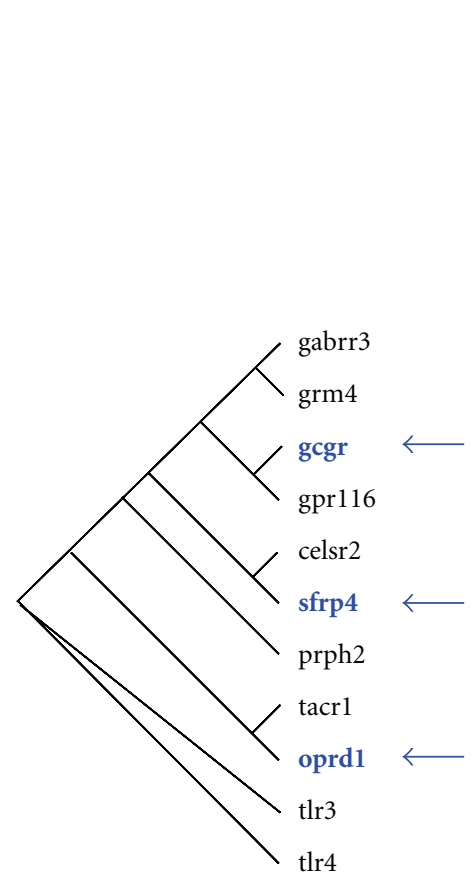

(c)

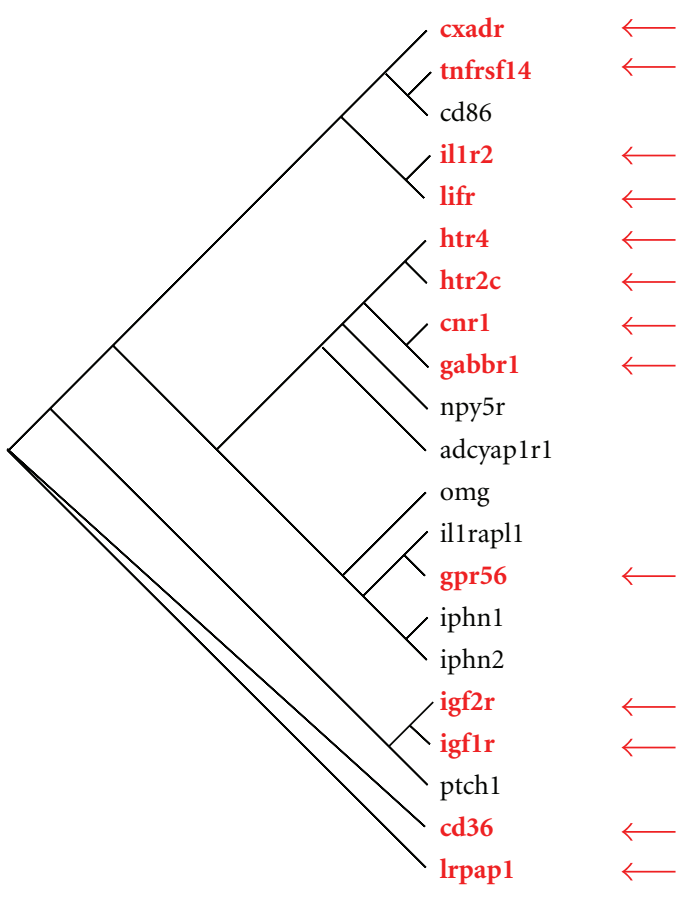

(d)

Figure 8: Alzheimer's disease correlation of control or 3xTgAD receptor-specific lipid raft proteins. (a) The histogram represents the number of receptor-specific raft proteins (Table 1: control, Table 2: 3xTgAD) that demonstrate an explicit LSI correlation to the term "Alzheimer's" for control (blue) or 3xTgAD (red) extracts. (b) The histogram depicts the cumulated LSI correlation scores for the Alzheimer's-related receptorspecific proteins identified in (a). panels (c) and (d) represent the phylogenetic dendrograms for the receptor-specific raft proteins linked to the interrogation term "Alzheimer's" from control (c) or 3xTgAD (d) datasets. The proteins highlighted in red or blue and indicated by an arrow were specifically clustered into the highest scoring protein interaction networks for control (Figure 7(b)) or 3xTgAD (Figure 7(c)) samples.

un-biased informatic processes, we were able to identify specific lipid raft involvement in altered pathways that can control multiple degenerative mechanisms, for example, attenuation of the $\mathrm{Wnt} / \beta$-catenin signaling pathway, profound reduction of the important Nrf2 stress response pathway (Figure 4(d)), the loss of neurogenesis association, and the important involvement of synaptic GPCR-systems (Figure 6) in the 3xTgAD mice [36-38].

Elucidation of the crucial signaling relationships in this degenerative disorder, and the identity of the proteins that mediate them, could lead to the more rational development of novel therapeutics for Alzheimer's disease. Using our informatic receptor-targeted approach, we were also able to reinforce the validity of our discovery process by also identifying the importance of energy-related insulin/insulinlike growth factor (IGF) signaling in $\mathrm{AD}$ (Figures 3(j), $3(\mathrm{n}), 5$, and $8(\mathrm{~d}))$ that has recently become more widely appreciated by other researchers [39-42]. In addition to IGF receptor activity, we noted a potential implication of the presynaptic latrophilins (lphn) in AD pathophysiology (Figure $8(d)$ ). These unique receptors form the high affinity target of $\alpha$-latrotoxin and may be able to integrate presynaptic calcium regulation with an ability to potentially physically interact with the postsynaptic neuron [43]. Another receptor signaling system that our analysis revealed to possess a potential role in $\mathrm{AD}$ is the Sonic Hedgehog (Shh) systems (Figures 5 and $8(\mathrm{~d})$ ). With a specific correlation to AD pathophysiology, it has been demonstrated that Shh can act in a synergistic manner with nerve growth factor to act on central nervous system cholinergic neurons [44], and cholinergic insufficiency has been strongly associated with cognitive decline and Alzheimer's-related pathophysiology [45]. 


\section{Conclusions}

The combined use of broad range proteomic analysis, with sequential multidimensional informatic analysis enables the determination of important correlations between pathophysiology and functional protein differences. Using discrete proteomes, that is, lipid raft extracts, versus whole-cell/tissue proteomes, facilitates an improved ability to understand the complex interactivity between transmembrane receptor protein systems in a disease setting. The appreciation and clustering of protein datasets into coherent groups greatly increases our capacity to focus upon potentially important therapeutic target networks. The knowledge gained of protein activity networks may greatly assist in the future development of network-targeting therapeutics that possess a multidimensional efficacy at several interacting proteins.

\section{Appendices}

\section{A.}

Lipid raft-derived extracted proteins from control mouse 16-month-old cortical tissue. Specific proteins isolated from control mouse cortex were identified with multidimensional protein identification technology (MudPIT) LC MS/MS. Each protein was identified in at least two out of three experimental replicate animals and from at least two isolated peptides per protein. The following are the protein symbol and its corresponding definitions.

Atm: ataxia telangiectasia mutated

Bcat1: branched chain aminotransferase 1, cytosolic

Bcl11a: B-cell CLL/lymphoma 11A (zinc finger protein)

Bnip1: BCL2/adenovirus E1B $19 \mathrm{kDa}$ interacting protein 1

Brd2: bromodomain containing 2

Bteb1: Kruppel-like factor 9

Bzrap1: benzodiazepine receptor (peripheral) associated protein 1

C3: complement component 3

C5: complement component 5

Cacnala: calcium channel, voltage-dependent, P/Q type, alpha $1 \mathrm{~A}$ subunit

Cacna1c: calcium channel, voltage-dependent, L type, alpha $1 \mathrm{C}$ subunit

Cacna2d2: calcium channel, voltage-dependent, alpha $2 /$ delta subunit 2

Cadps: Ca++-dependent secretion activator

Calb1: calbindin 1, $28 \mathrm{kDa}$

Calb2: calbindin 2

Calm2: calmodulin 2 (phosphorylase kinase, delta)

Camkk1: calcium/calmodulin-dependent protein kinase kinase 1, alpha
Camkk2: calcium/calmodulin-dependent protein kinase kinase 2, beta

Cap1: CAP, adenylate cyclase-associated protein 1 (yeast)

Cast: calpastatin

Cat: catalase

Catsper2: cation channel, sperm associated 2

Cav1: caveolin 1, caveolae protein, $22 \mathrm{kDa}$

Cblb: Cas-Br-M (murine) ecotropic retroviral transforming sequence $b$

Cbp: opsin 1 (cone pigments), long-wave-sensitive

Cbr1: carbonyl reductase 1

Cbwd1: COBW domain containing 1

Cbx5: chromobox homolog 5 (HP1 alpha homolog, Drosophila)

Ccbl1: cysteine conjugate-beta lyase, cytoplasmic

Ccin: calicin

Ccl28: chemokine (C-C motif) ligand 28

Ccl4: chemokine (C-C motif) ligand 4

ccnb1: cyclin B1

Ccnc: cyclin C

Cct2: chaperonin containing TCP1, subunit 2 (beta)

Cct3: chaperonin containing TCP1, subunit 3 (gamma)

Cct4: chaperonin containing TCP1, subunit 4 (delta)

Cct5: chaperonin containing TCP1, subunit 5 (epsilon)

Cct6a: chaperonin containing TCP1, subunit 6A (zeta 1)

Cd1: ribosomal protein L5

Cd151: CD151 molecule (Raph blood group)

Cd200: CD200 molecule

Cd276: CD276 molecule

Cd2ap: CD2-associated protein

Cd4: CD4 molecule

Cdc10: septin 7

Cdc25b: cell division cycle 25 homolog B (S. pombe)

Cdc42: cell division cycle 42 (GTP binding protein, $25 \mathrm{kDa}$ )

Cdc42bpa: CDC42 binding protein kinase alpha (DMPK-like)

Cdc5l: CDC5 cell division cycle 5-like (S. pombe)

Cdh1: cadherin 1, type 1, E-cadherin (epithelial)

Cdh10: cadherin 10, type 2 (T2-cadherin)

Cdh2: cadherin 2, type 1, N-cadherin (neuronal)

Cdh4: cadherin 4, type 1, R-cadherin (retinal) 
Cdk2: cyclin-dependent kinase 2

Cdk5rap2: CDK5 regulatory subunit associated protein 2

Cdk5rap3: CDK5 regulatory subunit associated protein 3

Cdk6: cyclin-dependent kinase 6

Cdk7: cyclin-dependent kinase 7

Cdkl2: cyclin-dependent kinase-like 2 (CDC2-related kinase)

Cdkl3: cyclin-dependent kinase-like 3

Cdkn1b: cyclin-dependent kinase inhibitor 1B (p27, Kip1)

Cds1: CDP-diacylglycerol synthase (phosphatidate cytidylyltransferase) 1

Cdv1: intraflagellar transport 81 homolog (Chlamydomonas)

Cebpe: CCAAT/enhancer binding protein (C/EBP), epsilon

Cebpz: CCAAT/enhancer binding protein (C/EBP), zeta

Celsr2: cadherin, EGF LAG seven-pass G-type receptor 2 (flamingo homolog, Drosophila)

Celsr3: cadherin, EGF LAG seven-pass G-type receptor 3 (flamingo homolog, Drosophila)

Cend1: cell cycle exit and neuronal differentiation 1

Cenpf: centromere protein F, 350/400ka (mitosin)

Centa1: ArfGAP with dual PH domains 1

Centg1: ArfGAP with GTPase domain, ankyrin repeat and $\mathrm{PH}$ domain 2

Cep350: centrosomal protein $350 \mathrm{kDa}$

Ces1: carboxylesterase 1 (monocyte/macrophage serine esterase 1)

Ces3: carboxylesterase 3

Cetn2: centrin, EF-hand protein, 2

Cetn3: centrin, EF-hand protein, 3 (CDC31 homo$\log$, yeast)

Cfh: complement factor $\mathrm{H}$

Cfl1: cofilin 1 (nonmuscle)

Chd3: chromodomain helicase DNA binding protein 3

Chd4: chromodomain helicase DNA binding protein 4

Chga: chromogranin A (parathyroid secretory protein 1)

Chl1: cell adhesion molecule with homology to L1CAM (close homolog of L1)

Chmpla: chromatin modifying protein $1 \mathrm{~A}$

Chn2: chimerin (chimaerin) 2
Chrm5: cholinergic receptor, muscarinic 5

Chrnb3: cholinergic receptor, nicotinic, beta 3

Chrnb4: cholinergic receptor, nicotinic, beta 4

Chrng: cholinergic receptor, nicotinic, gamma

Chst7: carbohydrate ( $\mathrm{N}$-acetylglucosamine 6-O) sulfotransferase 7

Chx10: visual system homeobox 2

Cirbp: cold inducible RNA binding protein

Cit: citron (rho-interacting, serine/threonine kinase 21)

Cktsf1b1: gremlin 1, cysteine knot superfamily, homolog (Xenopus laevis)

Clasp2: cytoplasmic linker associated protein 2

Clcc1: chloride channel CLIC-like 1

Clcn7: chloride channel 7

Cldn1: claudin 1

Cldn19: claudin 19

Cldn4: claudin 4

CLEC10A: C-type lectin domain family 10, member A

Clecsf6: C-type lectin domain family 4, member A

Clic4: chloride intracellular channel 4

CLIP3: CAP-GLY domain containing linker protein 3

Clpb: ClpB caseinolytic peptidase B homolog (E. coli)

Clstn1: calsyntenin 1

Cltb: clathrin, light chain (Lcb)

Cndp2: CNDP dipeptidase 2 (metallopeptidase M20 family)

Cnga1: cyclic nucleotide gated channel alpha 1

Cnga4: cyclic nucleotide gated channel alpha 4

Cnksr2: connector enhancer of kinase suppressor of Ras 2

Cnot6: CCR4-NOT transcription complex, subunit 6 Cnp: $2^{\prime}, 3^{\prime}$-cyclic nucleotide $3^{\prime}$ phosphodiesterase

Cnr1: cannabinoid receptor 1 (brain)

Cntfr: ciliary neurotrophic factor receptor

Cntn1: contactin 1

Cog3: component of oligomeric golgi complex 3

Colm: gliomedin

Copa: coatomer protein complex, subunit alpha

Copb1: coatomer protein complex, subunit beta 1

COPB2: coatomer protein complex, subunit beta 2 (beta prime)

Cops4: COP9 constitutive photomorphogenic homolog subunit 4 (Arabidopsis) 
Cops8: COP9 constitutive photomorphogenic homolog subunit 8 (Arabidopsis)

Corola: coronin, actin binding protein, 1A

Coro7: coronin 7

Cp: ceruloplasmin (ferroxidase)

Cpe: carboxypeptidase E

Cplx1: complexin 1

Cpt1a: carnitine palmitoyltransferase 1A (liver)

Cpt2: carnitine palmitoyltransferase 2

Crabp2: cellular retinoic acid binding protein 2

Crhbp: corticotropin releasing hormone binding protein

Crip2: cysteine-rich protein 2

Crk: v-crk sarcoma virus CT10 oncogene homolog (avian)

Crkl: v-crk sarcoma virus CT10 oncogene homolog (avian)-like

Crmp1: collapsin response mediator protein 1

Crnkl1: crooked neck pre-mRNA splicing factor-like 1 (Drosophila)

Crp: C-reactive protein, pentraxin-related

Cryl: cryptochrome 1 (photolyase-like)

Crygc: crystallin, gamma C

Crygs: crystallin, gamma S

Crym: crystallin, mu

Cs: citrate synthase

Csf1: colony stimulating factor 1 (macrophage)

Csnk1e: casein kinase 1, epsilon

Csnk1g2: casein kinase 1, gamma 2

Csnk2a1: casein kinase 2, alpha 1 polypeptide

Csnk2b: casein kinase 2, beta polypeptide

Cspg2: versican

Cspg3: neurocan

Cspg5: chondroitin sulfate proteoglycan 5 (neuroglycan C)

Cspg6: structural maintenance of chromosomes 3

Cst6: cystatin E/M

Cstb: cystatin B (stefin B)

Ctf1: cardiotrophin 1

Cthrc1: collagen triple helix repeat containing 1

Ctla4: cytotoxic T-lymphocyte-associated protein 4

Ctnnb1: catenin (cadherin-associated protein), beta $1,88 \mathrm{kDa}$

Ctnnd2: catenin (cadherin-associated protein), delta 2 (neural plakophilin-related arm-repeat protein)

Ctrb: chymotrypsinogen B1
Ctrl: chymotrypsin-like

Ctsd: cathepsin D

Cttn: cortactin

Cttnbp2: cortactin binding protein 2

Cul1: cullin 1

Cul5: cullin 5

Cuta: cutA divalent cation tolerance homolog (E. coli)

Cutl1: cut-like homeobox 1

CX3CL1: chemokine (C-X3-C motif) ligand 1

Cxadr: coxsackie virus and adenovirus receptor

Cxcl2: chemokine (C-X-C motif) ligand 2

Cyb5: cytochrome b5 type A (microsomal)

Cyb5b: cytochrome b5 type B (outer mitochondrial membrane)

Cyb5r1: cytochrome b5 reductase 1

Cybb: cytochrome b-245, beta polypeptide

Cyln2: CAP-GLY domain containing linker protein 2

Cyp2s1: cytochrome P450, family 2, subfamily S, polypeptide 1

Cyr61: cysteine-rich, angiogenic inducer, 61

Dab2: disabled homolog 2, mitogen-responsive phosphoprotein (Drosophila)

Dab2ip: DAB2 interacting protein

Dad1: defender against cell death 1

Dag1: dystroglycan 1 (dystrophin-associated glycoprotein 1)

Dap: death-associated protein

Dapk3: death-associated protein kinase 3

Dbc1: deleted in bladder cancer 1

$\mathrm{DBH}$ : dopamine beta-hydroxylase (dopamine betamonooxygenase)

Dbn1: drebrin 1

Dbndd2: dysbindin (dystrobrevin binding protein 1) domain containing 2

Dbnl: drebrin-like

Dcd: dermcidin

Dci: dodecenoyl-Coenzyme A delta isomerase (3,2 trans-enoyl-Coenzyme A isomerase)

Dclk2: doublecortin-like kinase 2

Dclre1c: DNA cross-link repair 1C (PSO2 homolog, S. cerevisiae)

Dctn2: dynactin 2 (p50)

Ddah2: dimethylarginine dimethylaminohydrolase 2

Ddb1: damage-specific DNA binding protein 1, $127 \mathrm{kDa}$ 
Ddn: dendrin

Ddost: dolichyl-diphosphooligosaccharide-protein glycosyltransferase

Ddr2: discoidin domain receptor tyrosine kinase 2

Ddt: D-dopachrome tautomerase

Ddx1: DEAD (Asp-Glu-Ala-Asp) box polypeptide 1

Ddx17: DEAD (Asp-Glu-Ala-Asp) box polypeptide 17

Ddx47: DEAD (Asp-Glu-Ala-Asp) box polypeptide 47

Ddx5: DEAD (Asp-Glu-Ala-Asp) box polypeptide 5

Decr1: 2,4-dienoyl CoA reductase 1, mitochondrial

Des: desmin

Dgcr14: DiGeorge syndrome critical region gene 14

Dgkb: diacylglycerol kinase, beta $90 \mathrm{kDa}$

Dgkg: diacylglycerol kinase, gamma $90 \mathrm{kDa}$

Dhcr7: 7-dehydrocholesterol reductase

Dhodh: dihydroorotate dehydrogenase

Dhrs8: hydroxysteroid (17-beta) dehydrogenase 11

Dhx16: DEAH (Asp-Glu-Ala-His) box polypeptide 16

Dhx30: DEAH (Asp-Glu-Ala-His) box polypeptide 30

Dial: cytochrome b5 reductase 3

Diol: deiodinase, iodothyronine, type I

Disc1: disrupted in schizophrenia 1

Dkc1: dyskeratosis congenita 1, dyskerin

Dlat: dihydrolipoamide S-acetyltransferase

Dlc1: deleted in liver cancer 1

Dld: dihydrolipoamide dehydrogenase

Dlgap1: discs, large (Drosophila) homolog-associated protein 1

Dlgap2: discs, large (Drosophila) homolog-associated protein 2

Dlgap4: discs, large (Drosophila) homolog-associated protein 4

Dll1: delta-like 1 (Drosophila)

Dll3: delta-like 3 (Drosophila)

Dlx5: distal-less homeobox 5

Dmd: dystrophin

Dmn: synemin, intermediate filament protein

Dmrt1: doublesex and mab-3 related transcription factor 1

Dnah6: dynein, axonemal, heavy chain 6

Dnaja2: DnaJ (Hsp40) homolog, subfamily A, member 2
Dnch1: dynein, cytoplasmic 1, heavy chain 1

Dncl2a: dynein, light chain, roadblock-type 1

Dntt: deoxynucleotidyltransferase, terminal

Dock9: dedicator of cytokinesis 9

Dpp3: dipeptidyl-peptidase 3

Dpyd: dihydropyrimidine dehydrogenase

Dpysl3: dihydropyrimidinase-like 3

Dpysl4: dihydropyrimidinase-like 4

Drd1ip: calcyon neuron-specific vesicular protein

Drg1: developmentally regulated GTP binding protein 1

Drpla: atrophin 1

Dscam: Down syndrome cell adhesion molecule

Dscr111: regulator of calcineurin 2

Dtnb: dystrobrevin, beta

Duoxl: dual oxidase 1

Dusp12: dual specificity phosphatase 12

Dusp2: dual specificity phosphatase 2

Dvl1: dishevelled, dsh homolog 1 (Drosophila)

Dync1h1: dynein, cytoplasmic 1 , heavy chain 1

Dync1li2: dynein, cytoplasmic 1, light intermediate chain 2

Dyx1c1: dyslexia susceptibility 1 candidate 1

Eaf2: ELL associated factor 2

Ecel1: endothelin converting enzyme-like 1

Echdc1: enoyl Coenzyme A hydratase domain containing 1

Ecm1: extracellular matrix protein 1

Ecm2: extracellular matrix protein 2, female organ and adipocyte specific

Edf1: endothelial differentiation-related factor 1

Ednrb: endothelin receptor type B

Eef2: eukaryotic translation elongation factor 2

Efcbp 1: N-terminal EF-hand calcium binding protein 1

Efemp2: EGF-containing fibulin-like extracellular matrix protein 2

Efnb1: ephrin-B1

Egf: epidermal growth factor (beta-urogastrone)

Egfl3: multiple EGF-like-domains 6

Egfr: epidermal growth factor receptor (erythroblastic leukemia viral (v-erb-b) oncogene homolog, avian)

Ehd2: EH-domain containing 2

Ehd4: EH-domain containing 4

Eif2c2: eukaryotic translation initiation factor 2C, 2 
Eif5b: eukaryotic translation initiation factor $5 \mathrm{~B}$

Elac2: elaC homolog 2 (E. coli)

Elavl3: ELAV (embryonic lethal, abnormal vision, Drosophila)-like 3 (Hu antigen C)

ELK1: ELK1, member of ETS oncogene family

Elmo1: engulfment and cell motility 1

Emb: embigin homolog (mouse)

Emd: emerin

Eml5: echinoderm microtubule associated protein like 5

Enah: enabled homolog (Drosophila)

Enc1: ectodermal-neural cortex (with BTB-like domain)

Eno2: enolase 2 (gamma, neuronal)

Eno3: enolase 3 (beta, muscle)

Enpp2: ectonucleotide pyrophosphatase/phosphodiesterase 2

Enpp3: ectonucleotide pyrophosphatase/phosphodiesterase 3

Enpp5: ectonucleotide pyrophosphatase/phosphodiesterase 5 (putative function)

Ensa: endosulfine alpha

Entpd2: ectonucleoside triphosphate diphosphohydrolase 2

Entpd8: ectonucleoside triphosphate diphosphohydrolase 8

Epha3: EPH receptor A3

Epha5: EPH receptor A5

Epha7: EPH receptor A7

Ephb3: EPH receptor B3

Ephx1: epoxide hydrolase 1, microsomal (xenobiotic)

Epn1: epsin 1

Eprs: glutamyl-prolyl-tRNA synthetase

Eps811: EPS8-like 1

Erap1: endoplasmic reticulum aminopeptidase 1

Erbb3: v-erb-b2 erythroblastic leukemia viral oncogene homolog 3 (avian)

Erbb4: v-erb-a erythroblastic leukemia viral oncogene homolog 4 (avian)

Ercc5: excision repair cross-complementing rodent repair deficiency, complementation group 5

Erp29: endoplasmic reticulum protein 29

Esd: esterase D/formylglutathione hydrolase

Esr2: estrogen receptor 2 (ER beta)

Etfb: electron-transfer-flavoprotein, beta polypeptide Ets1: v-ets erythroblastosis virus E26 oncogene homolog 1 (avian)
Evl: Enah/Vasp-like

Exoc5: exocyst complex component 5

Exoc7: exocyst complex component 7

Ezr: ezrin

Fabp1: fatty acid binding protein 1, liver

Fabp2: fatty acid binding protein 2, intestinal

Fabp3: fatty acid binding protein 3, muscle and heart (mammary-derived growth inhibitor)

Fabp4: fatty acid binding protein 4, adipocyte

Fabp7: fatty acid binding protein 7 , brain

Fads1: fatty acid desaturase 1

Fads2: fatty acid desaturase 2

Faim: Fas apoptotic inhibitory molecule

Fancd2: Fanconi anemia, complementation group D2

Faslg: Fas ligand (TNF superfamily, member 6)

Fasn: fatty acid synthase

Fat: FAT tumor suppressor homolog 1 (Drosophila)

Fat3: FAT tumor suppressor homolog 3 (Drosophila)

Fbn1: fibrillin 1

Fbn2: fibrillin 2

Fbxw11: F-box and WD repeat domain containing 11

Fetub: fetuin B

FGA: fibrinogen alpha chain

Fgb: fibrinogen beta chain

Fgf18: fibroblast growth factor 18

Fgf23: fibroblast growth factor 23

Fgfr1: fibroblast growth factor receptor 1

Fgfr1op2: FGFR1 oncogene partner 2

Fgfr2: fibroblast growth factor receptor 2

Fgfr3: fibroblast growth factor receptor 3

Fhl1: four and a half LIM domains 1

Fignl1: fidgetin-like 1

Fip1l1: FIP1 like 1 (S. cerevisiae)

Fkbp14: FK506 binding protein 14, 22 kDa

Fkbp1b: FK506 binding protein 1B, $12.6 \mathrm{kDa}$

Fkbp5: FK506 binding protein 5

Flg: filaggrin

Flii: flightless I homolog (Drosophila)

Fmo3: flavin containing monooxygenase 3

Fnta: farnesyltransferase, CAAX box, alpha

Fos: FBJ murine osteosarcoma viral oncogene homo$\log$

Fpgt: fucose-1-phosphate guanylyltransferase

Freq: frequenin homolog (Drosophila) 
Fshr: follicle stimulating hormone receptor

Fthfd: aldehyde dehydrogenase 1 family, member L1

Fuca1: fucosidase, alpha-L-1, tissue

fut10: fucosyltransferase 10 (alpha $(1,3)$ fucosyltransferase)

Fxc1: fracture callus 1 homolog (rat)

Fxyd6: FXYD domain containing ion transport regulator 6

Fzd9: frizzled homolog 9 (Drosophila)

Gaa: glucosidase, alpha; acid

Gabarap: GABA(A) receptor-associated protein

Gabarapl2: GABA(A) receptor-associated proteinlike 2

Gabbr1: gamma-aminobutyric acid (GABA) B receptor, 1

Gabra3: gamma-aminobutyric acid (GABA) A receptor, alpha 3

Gabrg2: gamma-aminobutyric acid (GABA) A receptor, gamma 2

Gabrr3: gamma-aminobutyric acid (GABA) receptor, rho 3

Gadd45a: growth arrest and DNA-damage-inducible, alpha

Galc: galactosylceramidase

Galk1: galactokinase 1

Galt: galactose-1-phosphate uridylyltransferase

Gapdh: glyceraldehyde-3-phosphate dehydrogenase

Gars: glycyl-tRNA synthetase

Gas6: growth arrest-specific 6

Gas7: growth arrest-specific 7

Gata2: GATA binding protein 2

Gata3: GATA binding protein 3

GC: group-specific component (vitamin D binding protein)

Gc: group-specific component (vitamin D binding protein)

Gcgr: glucagon receptor

Gckr: glucokinase (hexokinase 4) regulator

Gclc: glutamate-cysteine ligase, catalytic subunit

Gcnt3: glucosaminyl (N-acetyl) transferase 3, mucin type

Gdap2: ganglioside induced differentiation associated protein 2

Gdil: GDP dissociation inhibitor 1

Gdi2: GDP dissociation inhibitor 2

Gfap: glial fibrillary acidic protein
Gfpt2: glutamine-fructose-6-phosphate transaminase 2

Gfra1: GDNF family receptor alpha 1

Gfra3: GDNF family receptor alpha 3

Ggt1: gamma-glutamyltransferase 1

Ggtl3: gamma-glutamyltransferase 7

Ggtla1: gamma-glutamyltransferase 5

Ghitm: growth hormone inducible transmembrane protein

Gif: gastric intrinsic factor (vitamin B synthesis)

Gipc1: GIPC PDZ domain containing family, member 1

Git1: G protein-coupled receptor kinase interacting ArfGAP 1

Gjal: gap junction protein, alpha $1,43 \mathrm{kDa}$

Gja10: gap junction protein, alpha $10,62 \mathrm{kDa}$

Gja4: gap junction protein, alpha $4,37 \mathrm{kDa}$

Gjb6: gap junction protein, beta $6,30 \mathrm{kDa}$

Gla: galactosidase, alpha

Gldc: glycine dehydrogenase (decarboxylating)

Glg1: golgi apparatus protein 1

Gli: GLI family zinc finger 1

Glo1: glyoxalase I

Glra2: glycine receptor, alpha 2

Glrx2: glutaredoxin 2

Glud1: glutamate dehydrogenase 1

Glul: glutamate-ammonia ligase (glutamine synthetase)

Gm2a: GM2 ganglioside activator

Gmcl1: germ cell-less homolog 1 (Drosophila)

Gmfb: glia maturation factor, beta

Gmfg: glia maturation factor, gamma

Gmpr2: guanosine monophosphate reductase 2

Gng7: guanine nucleotide binding protein ( $G$ protein), gamma 7

Gnpat: glyceronephosphate O-acyltransferase

Gns: glucosamine (N-acetyl)-6-sulfatase

Golga2: golgi autoantigen, golgin subfamily a, 2

Golgb1: golgin B1, golgi integral membrane protein

Golph3: golgi phosphoprotein 3 (coat-protein)

Golph4: golgi integral membrane protein 4

Gosr2: golgi SNAP receptor complex member 2

Got1: glutamic-oxaloacetic transaminase 1, soluble (aspartate aminotransferase 1)

Got2: glutamic-oxaloacetic transaminase 2, mitochondrial (aspartate aminotransferase 2) 
Gpam: glycerol-3-phosphate acyltransferase, mitochondrial

Gpc2: glypican 2

Gpi: glucose phosphate isomerase

Gpm6a: glycoprotein M6A

Gpr116: G protein-coupled receptor 116

Gpr141: G protein-coupled receptor 141

Gprc6a: G protein-coupled receptor, family C, group 6, member A

Gpsm1: G-protein signaling modulator 1 (AGS3-like, C. elegans)

Gpt: glutamic-pyruvate transaminase (alanine aminotransferase)

Gramd1a: GRAM domain containing 1A

Grasp: GRP1 (general receptor for phosphoinositides 1)-associated scaffold protein

Grem1: gremlin 1, cysteine knot superfamily, homo$\log$ (Xenopus laevis)

Gria1: glutamate receptor, ionotropic, AMPA 1

Grid2: glutamate receptor, ionotropic, delta 2

Grik1: glutamate receptor, ionotropic, kainate 1

Grik4: glutamate receptor, ionotropic, kainate 4

Grin2a: glutamate receptor, ionotropic, N-methyl Daspartate $2 \mathrm{~A}$

Grin2b: glutamate receptor, ionotropic, N-methyl Daspartate $2 \mathrm{~B}$

Grip1: glutamate receptor interacting protein 1

Gripap1: GRIP1 associated protein 1

Grk4: G protein-coupled receptor kinase 4

Grk5: G protein-coupled receptor kinase 5

Grk6: G protein-coupled receptor kinase 6

Grm4: glutamate receptor, metabotropic 4

Grm5: glutamate receptor, metabotropic 5

Grpel1: GrpE-like 1, mitochondrial (E. coli)

Gstk1: glutathione S-transferase kappa 1

Gstm2: glutathione S-transferase mu 2 (muscle)

Gsto1: glutathione S-transferase omega 1

Gtf2ird1: GTF2I repeat domain containing 1

Gtpbp4: GTP binding protein 4

Gucyla2: guanylate cyclase 1, soluble, alpha 2

Gucyla3: guanylate cyclase 1, soluble, alpha 3

Gucy1b2: guanylate cyclase 1, soluble, beta 2

Gucy2d: guanylate cyclase 2D, membrane (retinaspecific)

Gulp1: GULP, engulfment adaptor PTB domain containing 1
Haao: 3-hydroxyanthranilate 3,4-dioxygenase Itpka: inositol 1,4,5-trisphosphate 3-kinase A

Khdrbs2: KH domain containing, RNA binding, signal transduction associated 2

Kpnb1: karyopherin (importin) beta 1

Lamp2: lysosomal-associated membrane protein 2

Ncstn: nicastrin

Nedd4l: neural precursor cell expressed, developmentally down-regulated 4-like

Nip7: nuclear import 7 homolog (S. cerevisiae)

Nnat: neuronatin

Nos1: nitric oxide synthase 1 (neuronal)

Nox1: NADPH oxidase 1

Npepps: aminopeptidase puromycin sensitive

Nr1h3: nuclear receptor subfamily 1, group $H$, member 3

Nr1i2: nuclear receptor subfamily 1, group I, member 2

Nr6a1: nuclear receptor subfamily 6, group A, member 1

Nrcam: neuronal cell adhesion molecule

Nrd1: nardilysin ( $\mathrm{N}$-arginine dibasic convertase)

Ntn1: netrin 1

Nucb2: nucleobindin 2

Nudc: nuclear distribution gene C homolog (A. nidulans)

Nup35: nucleoporin $35 \mathrm{kDa}$

Nup54: nucleoporin $54 \mathrm{kDa}$

Nup98: nucleoporin $98 \mathrm{kDa}$

Oaz1: ornithine decarboxylase antizyme 1

Ocrl: oculocerebrorenal syndrome of Lowe

Oit3: oncoprotein induced transcript 3

Olfm2: olfactomedin 2

Opa1: optic atrophy 1 (autosomal dominant)

Oplah: 5-oxoprolinase (ATP-hydrolysing)

Oprd1: opioid receptor, delta 1

Orc4l: origin recognition complex, subunit 4-like (yeast)

Osmr: oncostatin $\mathrm{M}$ receptor

Oxr1: oxidation resistance 1

P2rx7: purinergic receptor $\mathrm{P} 2 \mathrm{X}$, ligand-gated ion channel, 7

P4hb: prolyl 4-hydroxylase, beta polypeptide

Pa2g4: proliferation-associated 2G4, $38 \mathrm{kDa}$

Pabpc4: poly(A) binding protein, cytoplasmic 4 (inducible form) 
Pace4: proprotein convertase subtilisin/kexin type 6

Pacs1: phosphofurin acidic cluster sorting protein 1

Pafah1b2: platelet-activating factor acetylhydrolase, isoform $\mathrm{Ib}$, subunit $2(30 \mathrm{kDa})$

Pafah1b3: platelet-activating factor acetylhydrolase, isoform Ib, subunit $3(29 \mathrm{kDa})$

Pak1: p21 protein (Cdc42/Rac)-activated kinase 1

Pak2: p21 protein (Cdc42/Rac)-activated kinase 2

Pak3: p21 protein (Cdc42/Rac)-activated kinase 3

Palm: paralemmin

Pamci: Ras association (RalGDS/AF-6) domain family (N-terminal) member 9

Panx1: pannexin 1

Panx2: pannexin 2

Pard3: par-3 partitioning defective 3 homolog (C. elegans)

Parg: poly (ADP-ribose) glycohydrolase

Park7: Parkinson disease (autosomal recessive, early onset) 7

Pawr: PRKC, apoptosis, WT1, regulator

Pax3: paired box 3

Pbp: phosphatidylethanolamine binding protein 1

Pc: pyruvate carboxylase

Pcca: propionyl Coenzyme A carboxylase, alpha polypeptide

Pcdh21: protocadherin 21

Pcdh7: protocadherin 7

Pcdha3: protocadherin alpha 3

Pcdhb1: protocadherin beta 1

Pck1: phosphoenolpyruvate carboxykinase 1 (soluble)

Pclo: piccolo (presynaptic cytomatrix protein)

Pcm1: pericentriolar material 1

Pcmt1: protein-L-isoaspartate (D-aspartate) Omethyltransferase

PCNT: pericentrin

Pcnx: pecanex homolog (Drosophila)

Pcnxl3: pecanex-like 3 (Drosophila)

Pcsk1: proprotein convertase subtilisin/kexin type 1

Pcsk1n: proprotein convertase subtilisin/kexin type 1 inhibitor

Pcsk5: proprotein convertase subtilisin/kexin type 5

Pcyox1: prenylcysteine oxidase 1

Pcyt1b: phosphate cytidylyltransferase 1, choline, beta

Pdap1: PDGFA associated protein 1
Pdc: phosducin

Pdcd2: programmed cell death 2

Pdcl: phosducin-like

Pde10a: phosphodiesterase 10A

Pde1a: phosphodiesterase 1A, calmodulin-dependent Pde1c: phosphodiesterase 1C, calmodulin-dependent $70 \mathrm{kDa}$

Pde4a: phosphodiesterase 4A, cAMP-specific (phosphodiesterase E2 dunce homolog, Drosophila)

Pde4b: phosphodiesterase 4B, cAMP-specific (phosphodiesterase E4 dunce homolog, Drosophila)

Pde4d: phosphodiesterase 4D, cAMP-specific (phosphodiesterase E3 dunce homolog, Drosophila)

Pde5a: phosphodiesterase 5A, cGMP-specific

Pde7a: phosphodiesterase 7A

Pde7b: phosphodiesterase 7B

Pdgfra: platelet-derived growth factor receptor, alpha polypeptide

Pdhb: pyruvate dehydrogenase (lipoamide) beta

Pdia3: protein disulfide isomerase family A, member 3

Pdia4: protein disulfide isomerase family A, member 4

Pdk4: pyruvate dehydrogenase kinase, isozyme 4

Pdlim7: PDZ and LIM domain 7 (enigma)

Pds5b: PDS5, regulator of cohesion maintenance, homolog B (S. cerevisiae)

Pdzd3: PDZ domain containing 3

Pepd: peptidase D

Per3: period homolog 3 (Drosophila)

Pex14: peroxisomal biogenesis factor 14

Pfkl: phosphofructokinase, liver

Pfkm: phosphofructokinase, muscle

Pfkp: phosphofructokinase, platelet

Pgam1: phosphoglycerate mutase 1 (brain)

PGAP1: post-GPI attachment to proteins 1

Pgd: phosphogluconate dehydrogenase

Pgf: placental growth factor

Pgk1: phosphoglycerate kinase 1

Pgm1: phosphoglucomutase 1

Pgr: progesterone receptor

Pgrmc1: progesterone receptor membrane component 1

Phactr1: phosphatase and actin regulator 1

Phactr3: phosphatase and actin regulator 3

Phb: prohibitin 
Phex: phosphate regulating endopeptidase homolog, X-linked

Phgdh: phosphoglycerate dehydrogenase

Phip: pleckstrin homology domain interacting protein

Phtf1: putative homeodomain transcription factor 1

Phyhipl: phytanoyl-CoA 2-hydroxylase interacting protein-like

Pi4ka: phosphatidylinositol 4-kinase, catalytic, alpha Picalm: phosphatidylinositol binding clathrin assembly protein

Pigq: phosphatidylinositol glycan anchor biosynthesis, class $\mathrm{Q}$

Pigs: phosphatidylinositol glycan anchor biosynthesis, class $\mathrm{S}$

Pik3cg: phosphoinositide-3-kinase, catalytic, gamma polypeptide

Pip5k1a: phosphatidylinositol-4-phosphate 5-kinase, type I, alpha

Pip5k1c: phosphatidylinositol-4-phosphate 5-kinase, type I, gamma

Pip5k2a: phosphatidylinositol-5-phosphate 4-kinase, type II, alpha

Pip5k2c: phosphatidylinositol-5-phosphate 4-kinase, type II, gamma

Pipox: pipecolic acid oxidase

Pitpn: phosphatidylinositol transfer protein, alpha

Pkd1: polycystic kidney disease 1 (autosomal dominant)

Pkia: protein kinase (cAMP-dependent, catalytic) inhibitor alpha

Pkm2: pyruvate kinase, muscle

Pla2g4c: phospholipase A2, group IVC (cytosolic, calcium-independent)

Plaa: phospholipase A2-activating protein

Plau: plasminogen activator, urokinase

Plcb1: phospholipase C, beta 1 (phosphoinositidespecific)

Plcl1: phospholipase C-like 1

Pld3: phospholipase D family, member 3

Plekha4: pleckstrin homology domain containing, family A (phosphoinositide binding specific) member 4

Plekhe1: $\mathrm{PH}$ domain and leucine rich repeat protein phosphatase 1

Plekhm1: pleckstrin homology domain containing, family M (with RUN domain) member 1

Plg: plasminogen

Plk1: polo-like kinase 1 (Drosophila)
Plrg1: pleiotropic regulator 1 (PRL1 homolog, Arabidopsis)

Plunc: palate, lung and nasal epithelium associated

Plvap: plasmalemma vesicle associated protein

Plxnb2: plexin B2

Pmpca: peptidase (mitochondrial processing) alpha

Pmvk: phosphomevalonate kinase

Pnliprp2: pancreatic lipase-related protein 2

Pnma1: paraneoplastic antigen MA1

Pno1: partner of NOB1 homolog (S. cerevisiae)

Pnpla2: patatin-like phospholipase domain containing 2

Pofut1: protein O-fucosyltransferase 1

Pon2: paraoxonase 2

Pon3: paraoxonase 3

Pop7: processing of precursor 7, ribonuclease P/MRP subunit (S. cerevisiae)

Por: P450 (cytochrome) oxidoreductase

Pou2f2: POU class 2 homeobox 2

Ppfia3: protein tyrosine phosphatase, receptor type, $\mathrm{f}$ polypeptide (PTPRF), interacting protein, alpha 3

Ppgb: cathepsin A

Ppib: peptidylprolyl isomerase B (cyclophilin B)

Ppid: peptidylprolyl isomerase D

Ppil3: peptidylprolyl isomerase (cyclophilin)-like 3

Ppm2c: pyruvate dehydrogenase phosphatase catalytic subunit 1

Ppp1r10: protein phosphatase 1, regulatory (inhibitor) subunit 10

Ppp1r14b: protein phosphatase 1, regulatory (inhibitor) subunit $14 \mathrm{~B}$

Ppp1r14d: protein phosphatase 1, regulatory (inhibitor) subunit $14 \mathrm{D}$

Ppplrla: protein phosphatase 1, regulatory (inhibitor) subunit $1 \mathrm{~A}$

Ppp1r7: protein phosphatase 1, regulatory (inhibitor) subunit 7

Ppp1r8: protein phosphatase 1, regulatory (inhibitor) subunit 8

Ppp1r9a: protein phosphatase 1, regulatory (inhibitor) subunit $9 \mathrm{~A}$

Ppp1r9b: protein phosphatase 1, regulatory (inhibitor) subunit $9 \mathrm{~B}$

Ppp2cb: protein phosphatase 2 (formerly 2A), catalytic subunit, beta isoform

Ppp2r1a: protein phosphatase 2 (formerly 2A), regulatory subunit $\mathrm{A}$, alpha isoform 
Ppp2r1b: protein phosphatase 2 (formerly 2A), regulatory subunit $\mathrm{A}$, beta isoform

Ppp2r2a: protein phosphatase 2 (formerly 2A), regulatory subunit $\mathrm{B}$, alpha isoform

PPP2R2B: protein phosphatase 2 (formerly 2A), regulatory subunit $\mathrm{B}$, beta isoform

Ppp2r5e: protein phosphatase 2, regulatory subunit B', epsilon isoform

Ppp3cc: protein phosphatase 3 (formerly 2B), catalytic subunit, gamma isoform

Ppp5c: protein phosphatase 5, catalytic subunit

Prdm4: PR domain containing 4

Prdx1: peroxiredoxin 1

Prep: prolyl endopeptidase

Prf1: perforin 1 (pore forming protein)

Prkaa2: protein kinase, AMP-activated, alpha 2 catalytic subunit

Prkaca: protein kinase, cAMP-dependent, catalytic, alpha

prkar1a: protein kinase, cAMP-dependent, regulatory, type I, alpha (tissue specific extinguisher 1)

Prkarla: protein kinase, cAMP-dependent, regulatory, type I, alpha (tissue specific extinguisher 1)

Prkar2a: protein kinase, cAMP-dependent, regulatory, type II, alpha

Prkar2b: protein kinase, cAMP-dependent, regulatory, type II, beta

Prkca: protein kinase $\mathrm{C}$, alpha

Prkcb1: protein kinase $\mathrm{C}$, beta

Prkci: protein kinase $\mathrm{C}$, iota

Prkcm: protein kinase D1

PRKCQ: protein kinase C, theta

Prkcz: protein kinase $\mathrm{C}$, zeta

Prkwnk1: WNK lysine deficient protein kinase 1

Prmt3: protein arginine methyltransferase 3

Prnp: prion protein

Prom2: prominin 2

Prph2: peripherin 2 (retinal degeneration, slow)

Prpsap1: phosphoribosyl pyrophosphate synthetaseassociated protein 1

Prpsap2: phosphoribosyl pyrophosphate synthetaseassociated protein 2

Prr3: proline rich 3

Prrx2: paired related homeobox 2

Prrxl1: dorsal root ganglia homeobox

Prss 1: protease, serine, 1 (trypsin 1)

Prss12: protease, serine, 12 (neurotrypsin, motopsin)
Prss15: lon peptidase 1, mitochondrial

Prtg: protogenin homolog (Gallus gallus)

Prx: periaxin

Psat1: phosphoserine aminotransferase 1

Psd: pleckstrin and Sec7 domain containing

Psd4: pleckstrin and Sec7 domain containing 4

Psmc1: proteasome (prosome, macropain) 26S subunit, ATPase, 1

Psmc3: proteasome (prosome, macropain) 26S subunit, ATPase, 3

Psmc5: proteasome (prosome, macropain) 26S subunit, ATPase, 5

Psmd1: proteasome (prosome, macropain) 26S subunit, non-ATPase, 1

Psme1: proteasome (prosome, macropain) activator subunit 1 (PA28 alpha)

Psme2: proteasome (prosome, macropain) activator subunit 2 (PA28 beta)

Psme3: proteasome (prosome, macropain) activator subunit 3 (PA28 gamma; Ki)

Psph: phosphoserine phosphatase

Ptbp1: polypyrimidine tract binding protein 1

Ptbp2: polypyrimidine tract binding protein 2

Ptger2: prostaglandin E receptor 2 (subtype EP2), $53 \mathrm{kDa}$

Ptgis: prostaglandin I2 (prostacyclin) synthase

Ptgs1: prostaglandin-endoperoxide synthase 1 (prostaglandin $\mathrm{G} / \mathrm{H}$ synthase and cyclooxygenase)

Pth: parathyroid hormone

Pthr2: parathyroid hormone 2 receptor

Ptk2: PTK2 protein tyrosine kinase 2

Ptn: pleiotrophin

Ptov1: prostate tumor overexpressed 1

Ptp4a1: protein tyrosine phosphatase type IVA, member 1

Ptpn11: protein tyrosine phosphatase, non-receptor type 11

Rnmt: RNA (guanine-7-) methyltransferase

Rraga: Ras-related GTP binding A

Rtn4: reticulon 4

Ryr3: ryanodine receptor 3

Scal: ataxin 1

Sca10: ataxin 10

Scfd1: sec1 family domain containing 1

Scgb3a2: secretoglobin, family $3 \mathrm{~A}$, member 2

Scn1a: sodium channel, voltage-gated, type I, alpha subunit 
Scn2a1: sodium channel, voltage-gated, type II, alpha subunit

Scp2: sterol carrier protein 2

Sdad1: SDA1 domain containing 1

Sdc2: syndecan 2

Sdcbp: syndecan binding protein (syntenin)

Sdfr1: neuroplastin

Sdha: succinate dehydrogenase complex, subunit A, flavoprotein (Fp)

Sec1113: SEC11 homolog C (S. cerevisiae)

Sec3111: SEC31 homolog A (S. cerevisiae)

Sectm1: secreted and transmembrane 1

Sema6c: sema domain, transmembrane domain (TM), and cytoplasmic domain, (semaphorin) 6C

Sephs1: selenophosphate synthetase 1

Serinc3: serine incorporator 3

Serpinb3: serpin peptidase inhibitor, clade B (ovalbumin), member 3

Serpine2: serpin peptidase inhibitor, clade E (nexin, plasminogen activator inhibitor type 1), member 2

Sez6: seizure related 6 homolog (mouse)

Sf3b1: splicing factor $3 \mathrm{~b}$, subunit 1, $155 \mathrm{kDa}$

Sfpq: splicing factor proline/glutamine-rich (polypyrimidine tract binding protein associated)

Sfrp4: secreted frizzled-related protein 4

Sfrs10: transformer 2 beta homolog (Drosophila)

Sfrs8: splicing factor, arginine/serine-rich 8 (suppressor-of-white-apricot homolog, Drosophila)

Sfrs9: splicing factor, arginine/serine-rich 9

Sftpb: surfactant protein B

Sfxn3: sideroflexin 3

Sfxn5: sideroflexin 5

Sgca: sarcoglycan, alpha $(50 \mathrm{kDa}$ dystrophin-associated glycoprotein)

Sgtb: small glutamine-rich tetratricopeptide repeat (TPR)-containing, beta

Sh2d4a: SH2 domain containing 4A

Sh3bp4: SH3-domain binding protein 4

Sh3bp5: SH3-domain binding protein 5 (BTK-associated)

Sh3gl3: SH3-domain GRB2-like 3

Sh3glb2: SH3-domain GRB2-like endophilin B2

Shank1: SH3 and multiple ankyrin repeat domains 1

Shank2: SH3 and multiple ankyrin repeat domains 2

Shc1: SHC (Src homology 2 domain containing) transforming protein 1

Shh: sonic hedgehog homolog (Drosophila)
Shoc2: soc-2 suppressor of clear homolog (C. elegans)

Siat7A: ST6 (alpha-N-acetyl-neuraminyl-2,3-betagalactosyl-1,3)-N-acetylgalactosaminide alpha-2,6sialyltransferase 1

Sipa1: signal-induced proliferation-associated 1

Sipa112: signal-induced proliferation-associated 1 like 2

Sipa1l3: signal-induced proliferation-associated 1 like 3

Sirpa: signal-regulatory protein alpha

Sirt2: sirtuin (silent mating type information regulation 2 homolog) 2 (S. cerevisiae)

Sirt5: sirtuin (silent mating type information regulation 2 homolog) 5 (S. cerevisiae)

Slc11a2: solute carrier family 11 (proton-coupled divalent metal ion transporters), member 2

Slc12a1: solute carrier family 12 (sodium/potassium/chloride transporters), member 1

Slc12a5: solute carrier family 12 (potassium-chloride transporter), member 5

Slco3a1: solute carrier organic anion transporter family, member 3A1

Slit1: slit homolog 1 (Drosophila)

Slit2: slit homolog 2 (Drosophila)

Slit3: slit homolog 3 (Drosophila)

Slk: STE20-like kinase (yeast)

Slu7: SLU7 splicing factor homolog (S. cerevisiae)

Smad1: SMAD family member 1

Smarca2: SWI/SNF related, matrix associated, actin dependent regulator of chromatin, subfamily a, member 2

Smc1l1: structural maintenance of chromosomes 1A Smo: smoothened homolog (Drosophila)

Smoc1: SPARC related modular calcium binding 1

Smu1: smu-1 suppressor of mec-8 and unc-52 homolog (C. elegans)

Sn: sialic acid binding Ig-like lectin 1, sialoadhesin

Snail: snail homolog 1 (Drosophila)

Snai2: snail homolog 2 (Drosophila)

Snap23: synaptosomal-associated protein, $23 \mathrm{kDa}$

Snap25: synaptosomal-associated protein, $25 \mathrm{kDa}$

Snap91: synaptosomal-associated protein, $91 \mathrm{kDa}$ homolog (mouse)

Snca: synuclein, alpha (non A4 component of amyloid precursor)

Sncb: synuclein, beta 
Snd1: staphylococcal nuclease and tudor domain containing 1

Snph: syntaphilin

Snrk: SNF related kinase

Snx17: sorting nexin 17

Snx25: sorting nexin 25

Snx3: sorting nexin 3

Soat1: sterol O-acyltransferase 1

Socs 1: suppressor of cytokine signaling 1

SOD1: superoxide dismutase 1, soluble

Sod2: superoxide dismutase 2, mitochondrial

Sord: sorbitol dehydrogenase

Sox10: SRY (sex determining region Y)-box 10

Sp2: Sp2 transcription factor

Sparc: secreted protein, acidic, cysteine-rich (osteonectin)

Spata20: spermatogenesis associated 20

Spint1: serine peptidase inhibitor, Kunitz type 1

Spp1: secreted phosphoprotein 1

Spsb4: splA/ryanodine receptor domain and SOCS box containing 4

Sqle: squalene epoxidase

Src: v-src sarcoma (Schmidt-Ruppin A-2) viral oncogene homolog (avian)

Srd5a2: steroid-5-alpha-reductase, alpha polypeptide 2

Srebf1: sterol regulatory element binding transcription factor 1

Srprb: signal recognition particle receptor, B subunit Ssb: Sjogren syndrome antigen B (autoantigen La)

Ssr4: signal sequence receptor, delta (translocon-associated protein delta)

Ssrp1: structure specific recognition protein 1

Sstr2: somatostatin receptor 2

Ssx2ip: synovial sarcoma, $\mathrm{X}$ breakpoint 2 interacting protein

St14: suppression of tumorigenicity 14 (colon carcinoma)

St18: suppression of tumorigenicity 18 (breast carcinoma) (zinc finger protein)

St3gal1: ST3 beta-galactoside alpha-2,3-sialyltransferase 1

St6gal2: ST6 beta-galactosamide alpha-2,6-sialyltransferase 2

St7: suppression of tumorigenicity 7

St7l: suppression of tumorigenicity 7 like

Star: steroidogenic acute regulatory protein
Stard3nl: STARD3 N-terminal like

Stat1: signal transducer and activator of transcription $1,91 \mathrm{kDa}$

Stat3: signal transducer and activator of transcription 3 (acute-phase response factor)

Stau1: staufen, RNA binding protein, homolog 1 (Drosophila)

Stch: heat shock protein $70 \mathrm{kDa}$ family, member 13

Ste: sulfotransferase family $1 \mathrm{E}$, estrogen-preferring, member 1

Stim2: stromal interaction molecule 2

Stip1: stress-induced-phosphoprotein 1

Stk10: serine/threonine kinase 10

Stk11: serine/threonine kinase 11

Stmn1: stathmin 1

Stmn2: stathmin-like 2

Stmn3: stathmin-like 3

Stmn4: stathmin-like 4

Stnl: eukaryotic translation elongation factor 1 alpha 2

Stom: stomatin

Strbp: spermatid perinuclear RNA binding protein

Strn: striatin, calmodulin binding protein

Stx12: syntaxin 12

Stxla: syntaxin 1A (brain)

Stx4a: syntaxin 4

Stx5a: syntaxin 5

Stx6: syntaxin 6

Stx8: syntaxin 8

Stxbp3: syntaxin binding protein 3

Stxbp5: syntaxin binding protein 5 (tomosyn)

Suclg1: succinate-CoA ligase, alpha subunit

Sult1a1: sulfotransferase family, cytosolic, 1A, phenol-preferring, member 1

Sult1c1: sulfotransferase family, cytosolic, 1C, member 2

Sult1c2: sulfotransferase family, cytosolic, 1C, member 2

Sult1e1: sulfotransferase family 1E, estrogenpreferring, member 1

Sult4a1: sulfotransferase family $4 \mathrm{~A}$, member 1

Supv311: suppressor of var1, 3-like 1 (S. cerevisiae)

Surf6: surfeit 6

Suv420h2: suppressor of variegation 4-20 homolog 2 (Drosophila)

Sv2a: synaptic vesicle glycoprotein $2 \mathrm{~A}$ 
Sv2b: synaptic vesicle glycoprotein $2 \mathrm{~B}$

Sycp 1: synaptonemal complex protein 1

Sycp2: synaptonemal complex protein 2

Syn1: synapsin I

Syn2: synapsin II

Syn3: synapsin III

Syngap1: synaptic Ras GTPase activating protein 1 homolog (rat)

Synj1: synaptojanin 1

Synj2: synaptojanin 2

Synj2bp: synaptojanin 2 binding protein

Synpo: synaptopodin

Sypl: synaptophysin-like 1

Syt11: synaptotagmin XI

Syt12: synaptotagmin XII

Syt5: synaptotagmin V

Syt6: synaptotagmin VI

Syt8: synaptotagmin VIII

Tacc1: transforming, acidic coiled-coil containing protein 1

Tacc2: transforming, acidic coiled-coil containing protein 2

Tacr1: tachykinin receptor 1

Taf1c: TATA box binding protein (TBP)-associated factor, RNA polymerase I, C, $110 \mathrm{kDa}$

Taf9l: TAF9B RNA polymerase II, TATA box binding protein (TBP)-associated factor, $31 \mathrm{kDa}$

Tagln: transgelin

Tagln3: transgelin 3

Taldo1: transaldolase 1

Taok1: TAO kinase 1

Tapbp: TAP binding protein (tapasin)

Tars: threonyl-tRNA synthetase

Tas2r41: taste receptor, type 2, member 41

Tat: tyrosine aminotransferase

Tax1bp1: Tax1 (human T-cell leukemia virus type I) binding protein 1

Tbc1d10b: TBC1 domain family, member 10B

Tbca: tubulin folding cofactor A

Tbx2: T-box 2

Tcirg1: T-cell, immune regulator 1, ATPase, $\mathrm{H}+$ transporting, lysosomal V0 subunit A3

Tcn2: transcobalamin II; macrocytic anemia

Tcp1: t-complex 1

Tcra: T cell receptor alpha locus

Tdg: thymine-DNA glycosylase
Tdrd7: tudor domain containing 7

Tead1: TEA domain family member 1 (SV40 transcriptional enhancer factor)

Tegt: transmembrane BAX inhibitor motif containing 6

Tg: thyroglobulin

Tgfb 1: transforming growth factor, beta 1

Tgfbr3: transforming growth factor, beta receptor III Tgm1: transglutaminase 1 (K polypeptide epidermal type I, protein-glutamine-gamma-glutamyltransferase)

Tgm2: transglutaminase 2 (C polypeptide, proteinglutamine-gamma-glutamyltransferase)

Thbd: thrombomodulin

Thbs4: thrombospondin 4

Thpo: thrombopoietin

Thra: thyroid hormone receptor, alpha (erythroblastic leukemia viral (v-erb-a) oncogene homolog, avian)

Thrap3: thyroid hormone receptor associated protein 3

Thrb: thyroid hormone receptor, beta (erythroblastic leukemia viral (v-erb-a) oncogene homolog 2, avian)

Thy1: Thy-1 cell surface antigen $j$ list-item $i j l a b e l / i$

Tigd3: tigger transposable element derived 3

Timm44: translocase of inner mitochondrial membrane 44 homolog (yeast)

Timm8a: translocase of inner mitochondrial membrane 8 homolog A (yeast)

Tinag: tubulointerstitial nephritis antigen

Tjp1: tight junction protein 1 (zona occludens 1)

Tjp2: tight junction protein 2 (zona occludens 2)

Tkt: transketolase

Tle3: transducin-like enhancer of split 3 (E(sp1) homolog, Drosophila)

Tlr3: toll-like receptor 3

Tlr4: toll-like receptor 4

Tlr5: toll-like receptor 5

Tlr6: toll-like receptor 6

Tlr9: toll-like receptor 9

Tm4sf8: tetraspanin 3

Tm9sf2: transmembrane 9 superfamily member 2

Tm9sf3: transmembrane 9 superfamily member 3

Tmcol: transmembrane and coiled-coil domains 1

Tmeff1: transmembrane protein with EGF-like and two follistatin-like domains 1

Tmem27: transmembrane protein 27 
Tmf1: TATA element modulatory factor 1

Tmpo: thymopoietin

Tmsb10: thymosin beta 10

Tnfaip1: tumor necrosis factor, alpha-induced protein 1 (endothelial)

Tnnil: troponin I type 1 (skeletal, slow)

Tnni2: troponin I type 2 (skeletal, fast)

Tnnt3: troponin T type 3 (skeletal, fast)

Tob1: transducer of ERBB2, 1

Tob2: transducer of ERBB2, 2

Tom1: target of myb1 (chicken)

Tor3a: torsin family 3, member A

Tpbg: trophoblast glycoprotein

Tpd5212: tumor protein D52-like 2

Tph2: tryptophan hydroxylase 2

Tpm3: tropomyosin 3

Tpo: thyroid peroxidase

Tpst2: tyrosylprotein sulfotransferase 2

Tpt1: tumor protein, translationally-controlled 1

Tr4: nuclear receptor subfamily 2 , group C, member 2

Traf3ip1: TNF receptor-associated factor 3 interacting protein 1

Trak2: trafficking protein, kinesin binding 2

Trap1: TNF receptor-associated protein 1

Trib3: tribbles homolog 3 (Drosophila)

Trim13: tripartite motif-containing 13

Trim54: tripartite motif-containing 54

Trip 10: thyroid hormone receptor interactor 10

Trpc2: transient receptor potential cation channel, subfamily C, member 2 (pseudogene)

Trpc4: transient receptor potential cation channel, subfamily C, member 4

Trpc5: transient receptor potential cation channel, subfamily C, member 5

Trpc7: transient receptor potential cation channel, subfamily C, member 7

Trpv1: transient receptor potential cation channel, subfamily $\mathrm{V}$, member 1

Trpv4: transient receptor potential cation channel, subfamily $\mathrm{V}$, member 4

Tsc1: tuberous sclerosis 1

Tsc2: tuberous sclerosis 2

Tsg101: tumor susceptibility gene 101

Tsga10: testis specific, 10

Tsga10ip: testis specific, 10 interacting protein
Tshr: thyroid stimulating hormone receptor

Tsn: translin

Tsnax: translin-associated factor $\mathrm{X}$

Tst: thiosulfate sulfurtransferase (rhodanese)

Ttc1: tetratricopeptide repeat domain 1

Ttn: titin

Tub: tubby homolog (mouse)

Tuba1a: tubulin, alpha 1a

Tubb2b: tubulin, beta $2 \mathrm{~B}$

Tubb2c: tubulin, beta 2C

Tubb3: tubulin, beta 3

Tusc3: tumor suppressor candidate 3

Txn: thioredoxin

Txn2: thioredoxin 2

Txndc4: endoplasmic reticulum protein 44

Txnip: thioredoxin interacting protein

Txnl2: glutaredoxin 3

Txnrd1: thioredoxin reductase 1

Txnrd2: thioredoxin reductase 2

Ubc: ubiquitin C

Ube2e2: ubiquitin-conjugating enzyme E2E 2 (UBC4/5 homolog, yeast)

Ubl3: ubiquitin-like 3

Ubqln1: ubiquilin 1

Ubxd3: UBX domain protein 10

Uchl1: ubiquitin carboxyl-terminal esterase L1 (ubiquitin thiolesterase)

Ucp1: uncoupling protein 1 (mitochondrial, proton carrier)

Ucp2: uncoupling protein 2 (mitochondrial, proton carrier)

Ugtla1: UDP glucuronosyltransferase 1 family, polypeptide $\mathrm{A} 1$

Umod: uromodulin

Unc13a: unc-13 homolog A (C. elegans)

Unc13b: unc-13 homolog B (C. elegans)

Unc13c: unc-13 homolog C (C. elegans)

Unc5b: unc-5 homolog B (C. elegans)

Uqcrc2: ubiquinol-cytochrome c reductase core protein II

Uqcrfs 1: ubiquinol-cytochrome c reductase, Rieske iron-sulfur polypeptide 1

Uso1: USO1 homolog, vesicle docking protein (yeast)

Usp11: ubiquitin specific peptidase 11

Vamp1: vesicle-associated membrane protein 1 (synaptobrevin 1) 
Vamp2: vesicle-associated membrane protein 2 (synaptobrevin 2)

Vamp3: vesicle-associated membrane protein 3 (cellubrevin)

Vapa: VAMP (vesicle-associated membrane protein)associated protein $\mathrm{A}, 33 \mathrm{kDa}$

Vapb: VAMP (vesicle-associated membrane protein)associated protein $\mathrm{B}$ and $\mathrm{C}$

VCAN: versican

Vcan: versican

Vcp: valosin-containing protein

Vcpip1: valosin containing protein (p97)/p47 complex interacting protein 1

Vdac1: voltage-dependent anion channel 1

Vdac2: voltage-dependent anion channel 2

Vegfa: vascular endothelial growth factor A

Vgcnll: sodium leak channel, non-selective

Vgf: VGF nerve growth factor inducible

Vipr2: vasoactive intestinal peptide receptor 2

Vpreb1: pre-B lymphocyte 1

Vps33a: vacuolar protein sorting 33 homolog A (S. cerevisiae)

Vps33b: vacuolar protein sorting 33 homolog B (yeast)

Vps4a: vacuolar protein sorting 4 homolog A (S. cerevisiae)

Vsnl1: visinin-like 1

Vtn: vitronectin

Wars: tryptophanyl-tRNA synthetase

Wbp2: WW domain binding protein 2

Wdr10: intraflagellar transport 122 homolog (Chlamydomonas)

Wdr44: WD repeat domain 44

Wdr7: WD repeat domain 7

Wfs1: Wolfram syndrome 1 (wolframin)

Wifl: WNT inhibitory factor 1

Wipf1: WAS/WASL interacting protein family, member 1

Wnk4: WNK lysine deficient protein kinase 4

Wnt5a: wingless-type MMTV integration site family, member $5 \mathrm{~A}$

Wnt7a: wingless-type MMTV integration site family, member 7A

Xkr6: XK, Kell blood group complex subunit-related family, member 6

Xpnpep2: X-prolyl aminopeptidase (aminopeptidase P) 2, membrane-bound
Xpo1: exportin 1 (CRM1 homolog, yeast)

Xpo6: exportin 6

Xrcc1: X-ray repair complementing defective repair in Chinese hamster cells 1

Xrcc5: X-ray repair complementing defective repair in Chinese hamster cells 5

Xylt1: xylosyltransferase I

Yars2: tyrosyl-tRNA synthetase 2, mitochondrial

Yes1: v-yes-1 Yamaguchi sarcoma viral oncogene homolog 1

Ykt6: YKT6 v-SNARE homolog (S. cerevisiae)

Ywhab: tyrosine 3-monooxygenase/tryptophan 5monooxygenase activation protein, beta polypeptide

Yy1: YY1 transcription factor

Znf382: zinc finger protein 382

Zranb1: zinc finger, RAN-binding domain containing 1

Zwint: ZW10 interactor.

\section{B.}

Lipid raft-derived extracted proteins from Alzheimer's disease (3xTgAD) mouse 16-month-old cortical tissue. Specific proteins isolated from $3 \mathrm{xTgAD}$ mouse cortex were identified with multidimensional protein identification technology (MudPIT) LC MS/MS. Each protein was identified in at least two out of three experimental replicate animals and from at least two isolated peptides per protein. The following are the protein symbol and its corresponding definition.

Abca1: ATP-binding cassette, sub-family A (ABC1), member 1

ABCA7: ATP-binding cassette, sub-family A (ABC1), member 7

Abcb4: ATP-binding cassette, sub-family B (MDR/ TAP), member 4

Abcc1: ATP-binding cassette, sub-family C (CFTR/ MRP), member 1

Abcc2: ATP-binding cassette, sub-family C (CFTR/ MRP), member 2

Abcc4: ATP-binding cassette, sub-family C (CFTR/ MRP), member 4

Abi1: abl-interactor 1

Acadl: acyl-Coenzyme A dehydrogenase, long chain

Acat1: acetyl-Coenzyme A acetyltransferase 1

Acin 1: apoptotic chromatin condensation inducer 1

Acmsd: aminocarboxymuconate semialdehyde decarboxylase

Acp2: acid phosphatase 2, lysosomal

Acta1: actin, alpha 1, skeletal muscle 
Actb: actin, beta

Actn4: actinin, alpha 4

Acvr1: activin A receptor, type I

Adam2: ADAM metallopeptidase domain 2

Adam6: ADAM metallopeptidase domain 6 (pseudogene)

Adcy1: adenylate cyclase 1 (brain)

Adcy5: adenylate cyclase 5

Adcy8: adenylate cyclase 8 (brain)

Adcyap1: adenylate cyclase activating polypeptide 1 (pituitary)

Adcyap 1r1: adenylate cyclase activating polypeptide 1 (pituitary) receptor type I

Add1: adducin 1 (alpha)

Add2: adducin 2 (beta)

Adrbk1: adrenergic, beta, receptor kinase 1

Aes: amino-terminal enhancer of split

Agpat1: 1-acylglycerol-3-phosphate O-acyltransferase 1 (lysophosphatidic acid acyltransferase, alpha)

Agpat4: 1-acylglycerol-3-phosphate O-acyltransferase 4 (lysophosphatidic acid acyltransferase, delta)

Agrn: agrin

Ahi1: Abelson helper integration site 1

Ahr: aryl hydrocarbon receptor

Ahrr: aryl-hydrocarbon receptor repressor

Ak3: adenylate kinase 3

Akap12: A kinase (PRKA) anchor protein 12

Akap3: A kinase (PRKA) anchor protein 3

Akap4: A kinase (PRKA) anchor protein 4

Akap9: A kinase (PRKA) anchor protein (yotiao) 9

Alad: aminolevulinate, delta-, dehydratase

Aldh1a3: aldehyde dehydrogenase 1 family, member A3

Alox5: arachidonate 5-lipoxygenase

Ampd1: adenosine monophosphate deaminase 1 (isoform M)

Anxa3: annexin A3

Anxa6: annexin A6

Apex2: APEX nuclease (apurinic/apyrimidinic endonuclease) 2

Apob: apolipoprotein B (including $\mathrm{Ag}(\mathrm{x})$ antigen)

Apod: apolipoprotein D

App: amyloid beta (A4) precursor protein

Aps: kallikrein-related peptidase 3

Aqp3: aquaporin 3 (Gill blood group)

Aqp4: aquaporin 4
Aqp8: aquaporin 8

Arf1: ADP-ribosylation factor 1

Arf4: ADP-ribosylation factor 4

Arf6: ADP-ribosylation factor 6

Arfip1: ADP-ribosylation factor interacting protein 1 Arhgap20: Rho GTPase activating protein 20

Arhgap8: Rho GTPase activating protein 8

Arhgdia: Rho GDP dissociation inhibitor (GDI) alpha

Arhgef11: Rho guanine nucleotide exchange factor (GEF) 11

Arhgef12: Rho guanine nucleotide exchange factor (GEF) 12

Arhgef6: Rac/Cdc42 guanine nucleotide exchange factor (GEF) 6

Arnt: aryl hydrocarbon receptor nuclear translocator Arntl: aryl hydrocarbon receptor nuclear translocator-like

Arpp19: cAMP-regulated phosphoprotein, $19 \mathrm{kDa}$

Ascc1: activating signal cointegrator 1 complex subunit 1

Asz1: ankyrin repeat, SAM and basic leucine zipper domain containing 1

Atcay: ataxia, cerebellar, Cayman type

Atf3: activating transcription factor 3

Atm: ataxia telangiectasia mutated

Atpla1: ATPase, $\mathrm{Na}+/ \mathrm{K}+$ transporting, alpha 1 polypeptide

Atp1a4: ATPase, $\mathrm{Na}+/ \mathrm{K}+$ transporting, alpha 4 polypeptide

Atp1b1: ATPase, $\mathrm{Na}+/ \mathrm{K}+$ transporting, beta 1 polypeptide

ATP2B1: ATPase, $\mathrm{Ca}++$ transporting, plasma membrane 1

Atp2b1: ATPase, $\mathrm{Ca}++$ transporting, plasma membrane 1

Atp2b2: ATPase, $\mathrm{Ca}++$ transporting, plasma membrane 2

Atp5a1: ATP synthase, $\mathrm{H}+$ transporting, mitochondrial F1 complex, alpha subunit 1, cardiac muscle

Atp5b: ATP synthase, $\mathrm{H}+$ transporting, mitochondrial F1 complex, beta polypeptide

Atp5f1: ATP synthase, $\mathrm{H}+$ transporting, mitochondrial F0 complex, subunit B1

Atp5h: ATP synthase, $\mathrm{H}+$ transporting, mitochondrial F0 complex, subunit d

Atp5i: ATP synthase, $\mathrm{H}+$ transporting, mitochondrial F0 complex, subunit E 
Atp5j: ATP synthase, $\mathrm{H}+$ transporting, mitochondrial F0 complex, subunit F6

Atp6b2: ATPase, $\mathrm{H}+$ transporting, lysosomal $56 / 58 \mathrm{kDa}, \mathrm{V} 1$ subunit B2

Atp6l: ATPase, $\mathrm{H}+$ transporting, lysosomal $16 \mathrm{kDa}$, V0 subunit c

Atp6v0a1: ATPase, $\mathrm{H}+$ transporting, lysosomal V0 subunit a1

ATPBD1C: GPN-loop GTPase 3

Atxn3: ataxin 3

Avil: advillin

B3gat1: beta-1,3-glucuronyltransferase 1 (glucuronosyltransferase $\mathrm{P}$ )

Bace1: beta-site APP-cleaving enzyme 1

Basp1: brain abundant, membrane attached signal protein 1

Bat2: HLA-B associated transcript 2

Bat5: HLA-B associated transcript 5

Bax: BCL2-associated X protein

Bcl10: B-cell CLL/lymphoma 10

Begain: brain-enriched guanylate kinase-associated homolog (rat)

Bmp6: bone morphogenetic protein 6

Bnip1: BCL2/adenovirus E1B $19 \mathrm{kDa}$ interacting protein 1

Bnip3: BCL2/adenovirus E1B $19 \mathrm{kDa}$ interacting protein 3

Bpnt1: $3^{\prime}\left(2^{\prime}\right), 5^{\prime}$-bisphosphate nucleotidase 1

Brd2: bromodomain containing 2

Brd8: bromodomain containing 8

Bst1: bone marrow stromal cell antigen 1

Btg2: BTG family, member 2

Bzrap1: benzodiazepine receptor (peripheral) associated protein 1

c3orf6: coiled-coil domain containing 50

C4a: complement component 4A (Rodgers blood group)

c8b: complement component 8 , beta polypeptide

Cabcl: chaperone, $\mathrm{ABC} 1$ activity of bc1 complex homolog (S. pombe)

Cabin1: calcineurin binding protein 1

Cacnalg: calcium channel, voltage-dependent, $\mathrm{T}$ type, alpha $1 \mathrm{G}$ subunit

Cacna2d1: calcium channel, voltage-dependent, alpha 2/delta subunit 1

Cacna2d2: calcium channel, voltage-dependent, alpha 2 /delta subunit 2
Cacna2d3: calcium channel, voltage-dependent, alpha 2/delta subunit 3

Cacnb2: calcium channel, voltage-dependent, beta 2 subunit

Calb1: calbindin 1, $28 \mathrm{kDa}$

Calb2: calbindin 2

Cald1: caldesmon 1

calm2: calmodulin 2 (phosphorylase kinase, delta)

Calr: calreticulin

Camk2g: calcium/calmodulin-dependent protein kinase II gamma

Camkk1: calcium/calmodulin-dependent protein kinase kinase 1 , alpha

Camkv: CaM kinase-like vesicle-associated

Canx: calnexin

Cap1: CAP, adenylate cyclase-associated protein 1 (yeast)

Capn1: calpain 1, (mu/l) large subunit

Capn5: calpain 5

Capn6: calpain 6

Capzb: capping protein (actin filament) muscle Zline, beta

Card9: caspase recruitment domain family, member 9

Carhsp1: calcium regulated heat stable protein 1, $24 \mathrm{kDa}$

Caskin1: CASK interacting protein 1

Casp7: caspase 7, apoptosis-related cysteine peptidase Cast: calpastatin

Cbp: opsin 1 (cone pigments), long-wave-sensitive

Cbx3: chromobox homolog 3 (HP1 gamma homo$\log$, Drosophila)

Ccnd2: cyclin D2

Ccs: copper chaperone for superoxide dismutase

Cct2: chaperonin containing TCP1, subunit 2 (beta)

Cd2ap: CD2-associated protein

Cd36: CD36 molecule (thrombospondin receptor)

Cd86: CD86 molecule

Cdc25b: cell division cycle 25 homolog B (S. pombe)

Cdc42: cell division cycle 42 (GTP binding protein, $25 \mathrm{kDa})$

Cdc5l: CDC5 cell division cycle 5-like (S. pombe)

Cdh1: cadherin 1, type 1, E-cadherin (epithelial)

Cdh10: cadherin 10, type 2 (T2-cadherin)

Cdh13: cadherin 13, H-cadherin (heart)

Cdh2: cadherin 2, type 1, N-cadherin (neuronal) 
Cdk5rap2: CDK5 regulatory subunit associated protein 2

Cdkn1b: cyclin-dependent kinase inhibitor 1B (p27, Kip1)

Cds1: CDP-diacylglycerol synthase (phosphatidate cytidylyltransferase) 1

Cdv1: intraflagellar transport 81 homolog (Chlamydomonas)

Cebp: CCAAT/enhancer binding protein $(\mathrm{C} / \mathrm{EBP})$, alpha

Cend1: cell cycle exit and neuronal differentiation 1

Cenpc1: centromere protein $\mathrm{C} 1$

Cenpi: centromere protein I

Centa1: ArfGAP with dual PH domains 1

Ces1: carboxylesterase 1 (monocyte/macrophage serine esterase 1)

Cetn2: centrin, EF-hand protein, 2

Cfd: complement factor D (adipsin)

Cfl1: cofilin 1 (nonmuscle)

Cftr: cystic fibrosis transmembrane conductance regulator (ATP-binding cassette sub-family C, member 7)

Chgb: chromogranin B (secretogranin 1)

Chka: choline kinase alpha

Chm: choroideremia (Rab escort protein 1)

Chst10: carbohydrate sulfotransferase 10

Chx10: visual system homeobox 2

Cktsf1b1: gremlin 1, cysteine knot superfamily, homolog (Xenopus laevis)

Clcf1: cardiotrophin-like cytokine factor 1

Cldn18: claudin 18

Clic4: chloride intracellular channel 4

Clip3: CAP-GLY domain containing linker protein 3

Clta: clathrin, light chain (Lca)

Cltb: clathrin, light chain (Lcb)

Cltc: clathrin, heavy chain $(\mathrm{Hc})$

Clu: clusterin

Cnga1: cyclic nucleotide gated channel alpha 1

Cngb1: cyclic nucleotide gated channel beta 1

Cnot4: CCR4-NOT transcription complex, subunit 4

Cnr1: cannabinoid receptor 1 (brain)

Col5a3: collagen, type V, alpha 3

Copb1: coatomer protein complex, subunit beta 1

Cops4: COP9 constitutive photomorphogenic homolog subunit 4 (Arabidopsis)

Corola: coronin, actin binding protein, 1A
Cp: ceruloplasmin (ferroxidase)

Cpa2: carboxypeptidase A2 (pancreatic)

Cpt2: carnitine palmitoyltransferase 2

Creb1: cAMP responsive element binding protein 1

Crhbp: corticotropin releasing hormone binding protein

Crip2: cysteine-rich protein 2

Cript: cysteine-rich PDZ-binding protein

Crmp1: collapsin response mediator protein 1

Cry2: cryptochrome 2 (photolyase-like)

Csf1: colony stimulating factor 1 (macrophage)

Csh1: chorionic somatomammotropin hormone 1 (placental lactogen)

Csnk1e: casein kinase 1, epsilon

Csnk1g1: casein kinase 1, gamma 1

Csnk1g3: casein kinase 1, gamma 3

Cspg6: structural maintenance of chromosomes 3

Cst6: cystatin E/M

Cstb: cystatin B (stefin B)

Ctnnb1: catenin (cadherin-associated protein), beta $1,88 \mathrm{kDa}$

Cttn: cortactin

Cugbp2: CUG triplet repeat, RNA binding protein 2 Cul5: cullin 5

Cutl1: cut-like homeobox 1

Cxadr: coxsackie virus and adenovirus receptor

Cxcl10: chemokine (C-X-C motif) ligand 10

Cyb5r4: cytochrome b5 reductase 4

Cyln2: CAP-GLY domain containing linker protein 2 Cyp19a1: cytochrome P450, family 19, subfamily A, polypeptide 1

Cypla1: cytochrome P450, family 1, subfamily A, polypeptide 1

Cyp4x1: cytochrome P450, family 4, subfamily X, polypeptide 1

Dab2ip: DAB2 interacting protein

Dbn 1: drebrin 1

Dctn1: dynactin 1 (p150, glued homolog, Drosophila)

Dctn2: dynactin 2 (p50)

Dctn4: dynactin 4 (p62)

Dcxr: dicarbonyl/L-xylulose reductase

Ddah2: dimethylarginine dimethylaminohydrolase 2

Ddb1: damage-specific DNA binding protein 1, $127 \mathrm{kDa}$

Ddt: D-dopachrome tautomerase 
Ddx1: DEAD (Asp-Glu-Ala-Asp) box polypeptide 1

Ddx19: DEAD (Asp-Glu-Ala-As) box polypeptide 19B

Ddx27: DEAD (Asp-Glu-Ala-Asp) box polypeptide 27

Ddx5: DEAD (Asp-Glu-Ala-Asp) box polypeptide 5

Dedd: death effector domain containing

Degs1: degenerative spermatocyte homolog 1, lipid desaturase (Drosophila)

Des: desmin

Dgkg: diacylglycerol kinase, gamma $90 \mathrm{kDa}$

Dhcr7: 7-dehydrocholesterol reductase

Dhodh: dihydroorotate dehydrogenase

Dhx40: DEAH (Asp-Glu-Ala-His) box polypeptide 40

Dial: cytochrome b5 reductase 3

Disc1: disrupted in schizophrenia 1

Dkc1: dyskeratosis congenita 1 , dyskerin

Dlat: dihydrolipoamide S-acetyltransferase

Dlc1: deleted in liver cancer 1

Dlgap2: discs, large (Drosophila) homolog-associated protein 2

Dlgap4: discs, large (Drosophila) homolog-associated protein 4

Dll1: delta-like 1 (Drosophila)

Dnah7: dynein, axonemal, heavy chain 7

Dnah9: dynein, axonemal, heavy chain 9

Dnajc5: DnaJ (Hsp40) homolog, subfamily C, member 5

Dnch1: dynein, cytoplasmic 1 , heavy chain 1

Dnch2: dynein, cytoplasmic 2, heavy chain 1

Dnm1: dynamin 1

Dnmt1: DNA (cytosine-5-)-methyltransferase 1

Dnmt3a: DNA (cytosine-5-)-methyltransferase 3 alpha

Dntt: deoxynucleotidyltransferase, terminal

Dpp3: dipeptidyl-peptidase 3

Dpp6: dipeptidyl-peptidase 6

Dpyd: dihydropyrimidine dehydrogenase

Drd1ip: calcyon neuron-specific vesicular protein

Drg1: developmentally regulated GTP binding protein 1

Dtnb: dystrobrevin, beta

Dtnbp1: dystrobrevin binding protein 1

Duox1: dual oxidase 1

Dvl1: dishevelled, dsh homolog 1 (Drosophila)
Dyx1c1: dyslexia susceptibility 1 candidate 1

Eaf2: ELL associated factor 2

Echs1: enoyl Coenzyme A hydratase, short chain, 1, mitochondrial

Eef1g: eukaryotic translation elongation factor 1 gamma

Eef2: eukaryotic translation elongation factor 2

Eef2k: eukaryotic elongation factor-2 kinase

Efemp2: EGF-containing fibulin-like extracellular matrix protein 2

Egfr: epidermal growth factor receptor (erythroblastic leukemia viral (v-erb-b) oncogene homolog, avian)

Eif2ak3: eukaryotic translation initiation factor 2alpha kinase 3

Eif2b1: eukaryotic translation initiation factor 2B, subunit 1 alpha, $26 \mathrm{kDa}$

Eif2c2: eukaryotic translation initiation factor 2C, 2

Eif2s2: eukaryotic translation initiation factor 2, subunit 2 beta, $38 \mathrm{kDa}$

eif4a1: eukaryotic translation initiation factor $4 \mathrm{~A}$, isoform 1

Elavl3: ELAV (embryonic lethal, abnormal vision, Drosophila)-like 3 ( $\mathrm{Hu}$ antigen $\mathrm{C}$ )

ELK1: ELK1, member of ETS oncogene family

Emd: emerin

Eno2: enolase 2 (gamma, neuronal)

Enpp2: ectonucleotide pyrophosphatase/phosphodiesterase 2

Entpd8: ectonucleoside triphosphate diphosphohydrolase 8

Ephx2: epoxide hydrolase 2, cytoplasmic

Epn1: epsin 1

Erp29: endoplasmic reticulum protein 29

Esd: esterase D/formylglutathione hydrolase

Espn: espin

Esrrb: estrogen-related receptor beta

Etfa: electron-transfer-flavoprotein, alpha polypeptide

Etfb: electron-transfer-flavoprotein, beta polypeptide

Exoc7: exocyst complex component 7

Ezr: ezrin

F5: coagulation factor V (proaccelerin, labile factor)

Fabp 1: fatty acid binding protein 1, liver

Fancd2: Fanconi anemia, complementation group D2

Fat: FAT tumor suppressor homolog 1 (Drosophila) 
Fat2: FAT tumor suppressor homolog 2 (Drosophila)

Fat3: FAT tumor suppressor homolog 3 (Drosophila)

Fbn1: fibrillin 1

Fdps: farnesyl diphosphate synthase

Fgfr1op2: FGFR1 oncogene partner 2

Filip1: filamin A interacting protein 1

Fkbp1b: FK506 binding protein 1B, 12.6 kDa

Flg: filaggrin

Flot1: flotillin 1

Flot2: flotillin 2

Fosl1: FOS-like antigen 1

Fos12: FOS-like antigen 2

Fpgt: fucose-1-phosphate guanylyltransferase

Frap1: mechanistic target of rapamycin (serine/threonine kinase)

Freq: frequenin homolog (Drosophila)

Fyn: FYN oncogene related to SRC, FGR, YES

Gaa: glucosidase, alpha; acid

Gabbr1: gamma-aminobutyric acid (GABA) B receptor, 1

Gabra3: gamma-aminobutyric acid (GABA) A receptor, alpha 3

Gabre: gamma-aminobutyric acid (GABA) A receptor, epsilon

Gadd45a: growth arrest and DNA-damage-inducible, alpha

Galc: galactosylceramidase

Galk1: galactokinase 1

Galnt10: UDP-N-acetyl-alpha-D-galactosamine: polypeptide $\mathrm{N}$-acetylgalactosaminyltransferase 10

Gars: glycyl-tRNA synthetase

Gas7: growth arrest-specific 7

Gc: group-specific component (vitamin D binding protein)

Gdil: GDP dissociation inhibitor 1

Gdi2: GDP dissociation inhibitor 2

Gfap: glial fibrillary acidic protein

Gif: gastric intrinsic factor (vitamin B synthesis)

Gjb2: gap junction protein, beta 2, $26 \mathrm{kDa}$

Gla: galactosidase, alpha

Gldc: glycine dehydrogenase (decarboxylating)

Glra2: glycine receptor, alpha 2

Glut3: solute carrier family 2 (facilitated glucose transporter), member 3

Gmcl1: germ cell-less homolog 1 (Drosophila)

Gmfb: glia maturation factor, beta
Gna11: guanine nucleotide binding protein ( $\mathrm{G}$ protein), alpha 11 (Gq class)

Gnail: guanine nucleotide binding protein (G protein), alpha inhibiting activity polypeptide 1

Gnai2: guanine nucleotide binding protein (G protein), alpha inhibiting activity polypeptide 2

Gnaol: guanine nucleotide binding protein (G protein), alpha activating activity polypeptide $\mathrm{O}$

Gnaq: guanine nucleotide binding protein ( $\mathrm{G}$ protein), q polypeptide

Gnaz: guanine nucleotide binding protein (G protein), alpha z polypeptide

Gnb211: guanine nucleotide binding protein (G protein), beta polypeptide 2-like 1

Gnl3: guanine nucleotide binding protein-like 3 (nucleolar)

Gnpat: glyceronephosphate O-acyltransferase

Gpi: glucose phosphate isomerase

Gpm6a: glycoprotein M6A

Gpr1: G protein-coupled receptor 1

Gpr141: G protein-coupled receptor 141

Gpr56: G protein-coupled receptor 56

Gpsm1: G-protein signaling modulator 1 (AGS3-like, C. elegans)

Gpt2: glutamic pyruvate transaminase (alanine aminotransferase) 2

Grin1: glutamate receptor, ionotropic, N-methyl Daspartate 1

Grpel1: GrpE-like 1, mitochondrial (E. coli)

Gstm2: glutathione S-transferase mu 2 (muscle)

Gstm3: glutathione S-transferase mu 3 (brain)

Gucy2d: guanylate cyclase 2D, membrane (retinaspecific)

Gucy2f: guanylate cyclase 2F, retinal

H1f0: $\mathrm{H} 1$ histone family, member 0

H1f4: histone cluster 1, H1e

H2afy: H2A histone family, member Y

Haao: 3-hydroxyanthranilate 3,4-dioxygenase

Hap 1: huntingtin-associated protein 1

Hapln3: hyaluronan and proteoglycan link protein 3

Hbld2: iron-sulfur cluster assembly 1 homolog (S. cerevisiae)

Hck: hemopoietic cell kinase

Hcn1: hyperpolarization activated cyclic nucleotidegated potassium channel 1

Hdac6: histone deacetylase 6

Hdgf: hepatoma-derived growth factor (highmobility group protein 1-like) 
Hdlbp: high density lipoprotein binding protein

Hey1: hairy/enhancer-of-split related with YRPW motif 1

Hgf: hepatocyte growth factor (hepapoietin A; scatter factor)

Hip1r: huntingtin interacting protein 1 related

Hivep1: human immunodeficiency virus type I enhancer binding protein 1

Hk1: hexokinase 1

Hmga2: high mobility group AT-hook 2

Hmgcr: 3-hydroxy-3-methylglutaryl-Coenzyme A reductase

Hmgcs1: 3-hydroxy-3-methylglutaryl-Coenzyme A synthase 1 (soluble)

Hmgn3: high mobility group nucleosomal binding domain 3

Hn1: hematological and neurological expressed 1

Hnf4a: hepatocyte nuclear factor 4, alpha

Hnmt: histamine N-methyltransferase

Hnrnph1: heterogeneous nuclear ribonucleoprotein $\mathrm{H} 1(\mathrm{H})$

Hnrpa1: heterogeneous nuclear ribonucleoprotein A1

Hnrpk: heterogeneous nuclear ribonucleoprotein $\mathrm{K}$

Hnrpu: heterogeneous nuclear ribonucleoprotein $\mathrm{U}$ (scaffold attachment factor A)

hook3: hook homolog 3 (Drosophila)

Hps5: Hermansky-Pudlak syndrome 5

Hpse: heparanase

Hras1: v-Ha-ras Harvey rat sarcoma viral oncogene homolog

Hsd11b2: hydroxysteroid (11-beta) dehydrogenase 2

Hsd17B1: hydroxysteroid (17-beta) dehydrogenase 1

Hsf1: heat shock transcription factor 1

Hsp90ab1: heat shock protein $90 \mathrm{kDa}$ alpha (cytosolic), class B member 1

Hspa14: heat shock $70 \mathrm{kDa}$ protein 14

Hspe1: heat shock $10 \mathrm{kDa}$ protein 1 (chaperonin 10)

Htr2c: 5-hydroxytryptamine (serotonin) receptor 2C

Htr3a: 5-hydroxytryptamine (serotonin) receptor 3A

Htr4: 5-hydroxytryptamine (serotonin) receptor 4

Hyou1: hypoxia up-regulated 1

Id2: inhibitor of DNA binding 2, dominant negative helix-loop-helix protein

Idh1: isocitrate dehydrogenase 1 (NADP+), soluble

Igf1r: insulin-like growth factor 1 receptor

Igf2r: insulin-like growth factor 2 receptor
Ikbkap: inhibitor of kappa light polypeptide gene enhancer in B-cells, kinase complex-associated protein

IL1b: interleukin 1, beta

Il1r2: interleukin 1 receptor, type II

Il1 rapl1: interleukin 1 receptor accessory protein-like 1

Il6: interleukin 6 (interferon, beta 2)

Ilf3: interleukin enhancer binding factor $3,90 \mathrm{kDa}$

Inhbc: inhibin, beta $\mathrm{C}$

Inpp4a: inositol polyphosphate-4-phosphatase, type I, $107 \mathrm{kDa}$

Inpp4b: inositol polyphosphate-4-phosphatase, type II, $105 \mathrm{kDa}$

Inpp5d: inositol polyphosphate-5-phosphatase, $145 \mathrm{kDa}$

Irs1: insulin receptor substrate 1

Irs2: insulin receptor substrate 2

Isg20: interferon stimulated exonuclease gene $20 \mathrm{kDa}$

Itga6: integrin, alpha 6

Itm2c: integral membrane protein $2 \mathrm{C}$

Itpr2: inositol 1,4,5-triphosphate receptor, type 2

Itpr3: inositol 1,4,5-triphosphate receptor, type 3

Ivl: involucrin

Jag1: jagged 1 (Alagille syndrome)

Jak1: Janus kinase 1

Jak2: Janus kinase 2

Junb: jun B proto-oncogene

Kalrn: kalirin, RhoGEF kinase

Katna1: katanin p60 (ATPase-containing) subunit A 1

Kcnh1: potassium voltage-gated channel, subfamily $\mathrm{H}$ (eag-related), member 1

Kcnh2: potassium voltage-gated channel, subfamily $\mathrm{H}$ (eag-related), member 2

Kcnk2: potassium channel, subfamily K, member 2

Kcns1: potassium voltage-gated channel, delayedrectifier, subfamily $S$, member 1

Kcns3: potassium voltage-gated channel, delayedrectifier, subfamily S, member 3

Kcnt1: potassium channel, subfamily $\mathrm{T}$, member 1

Kif5a: kinesin family member $5 \mathrm{~A}$

Klc1: kinesin light chain 1

Klhl12: kelch-like 12 (Drosophila)

Kpnb1: karyopherin (importin) beta 1

Kras: v-Ki-ras2 Kirsten rat sarcoma viral oncogene homolog 
Lama5: laminin, alpha 5

Lancl1: LanC lantibiotic synthetase component Clike 1 (bacterial)

Lars: leucyl-tRNA synthetase

Lbp: lipopolysaccharide binding protein

Lcmt1: leucine carboxyl methyltransferase 1

Letm1: leucine zipper-EF-hand containing transmembrane protein 1

Lgil: leucine-rich, glioma inactivated 1

Lgr7: relaxin/insulin-like family peptide receptor 1

Lhfpl4: lipoma HMGIC fusion partner-like 4

Lhx3: LIM homeobox 3

Lifr: leukemia inhibitory factor receptor alpha

Lin10: chromosome 16 open reading frame 70

LMO7: LIM domain 7

Lphn1: latrophilin 1

Lphn2: latrophilin 2

Lpin 1: lipin 1

Lrpap 1: low density lipoprotein receptor-related protein associated protein 1

Lsamp: limbic system-associated membrane protein

Ltbp1: latent transforming growth factor beta binding protein 1

Luzp1: leucine zipper protein 1

Ly6g5b: lymphocyte antigen 6 complex, locus G5B

Lyar: Ly1 antibody reactive homolog (mouse)

LYL1: lymphoblastic leukemia derived sequence 1

Lyst: lysosomal trafficking regulator

Lzic: leucine zipper and CTNNBIP1 domain containing

M6pr: mannose-6-phosphate receptor (cation dependent)

Magi3: membrane associated guanylate kinase, WW and PDZ domain containing 3

Maoa: monoamine oxidase A

Map1lc3a: microtubule-associated protein 1 light chain 3 alpha

Map2: microtubule-associated protein 2

Map3k7ip2: mitogen-activated protein kinase kinase kinase 7 interacting protein 2

Map4: microtubule-associated protein 4

Mapk1: mitogen-activated protein kinase 1

Mapkapk2: mitogen-activated protein kinase-activated protein kinase 2

Mark1: MAP/microtubule affinity-regulating kinase 1
Mark3: MAP/microtubule affinity-regulating kinase 3

Matr3: matrin 3

Mcm7: minichromosome maintenance complex component 7

Mdga2: MAM domain containing glycosylphosphatidylinositol anchor 2

Mdh1: malate dehydrogenase 1, NAD (soluble)

Mecp2: methyl CpG binding protein 2 (Rett syndrome)

Mfap3: microfibrillar-associated protein 3

Mgat1: mannosyl (alpha-1,3-)-glycoprotein beta-1,2$\mathrm{N}$-acetylglucosaminyltransferase

Mgat5: mannosyl (alpha-1,6-)-glycoprotein beta-1,6$\mathrm{N}$-acetyl-glucosaminyltransferase

Mid1: midline 1 (Opitz/BBB syndrome)

Mkln1: muskelin 1, intracellular mediator containing kelch motifs

Mkrn2: makorin ring finger protein 2

Mlp: MARCKS-like 1

Mmp10: matrix metallopeptidase 10 (stromelysin 2)

Mnat1: ménage à trois homolog 1, cyclin $\mathrm{H}$ assembly factor (Xenopus laevis)

Mpdz: multiple PDZ domain protein

Mpi: mannose phosphate isomerase

Mpo: myeloperoxidase

mpp7: membrane protein, palmitoylated 7 (MAGUK p55 subfamily member 7 )

mre11: MRE11 meiotic recombination 11 homolog A (S. cerevisiae)

Mrpl38: mitochondrial ribosomal protein L38

Mrp19: mitochondrial ribosomal protein L9

Mrps9: mitochondrial ribosomal protein S9

Msn: moesin

Mtap: methylthioadenosine phosphorylase

Mtdh: metadherin

Mtmr3: myotubularin-related protein 3

Mtr: 5-methyltetrahydrofolate-homocysteine methyltransferase

Mtus1: microtubule-associated tumor suppressor 1

Mx1: myxovirus (influenza virus) resistance 1, interferon-inducible protein p78 (mouse)

Mybpc1: myosin binding protein C, slow type

myc: v-myc myelocytomatosis viral oncogene homo$\log$ (avian)

Mycbpap: MYCBP associated protein

Myh10: myosin, heavy chain 10, nonmuscle 
Myh6: myosin, heavy chain 6, cardiac muscle, alpha

Myh9: myosin, heavy chain 9, nonmuscle

Myola: myosin IA

Myole: myosin IE

Myo5a: myosin VA (heavy chain 12, myoxin)

Myo5b: myosin VB

Myo7a: myosin VIIA

Myo9a: myosin IXA

Myom 1: myomesin 1, $185 \mathrm{kDa}$

Nab1: NGFI-A binding protein 1 (EGR1 binding protein 1)

Nab2: NGFI-A binding protein 2 (EGR1 binding protein 2)

Naca: nascent polypeptide-associated complex alpha subunit

Naglu: $\mathrm{N}$-acetylglucosaminidase, alpha-

Nap1l3: nucleosome assembly protein 1-like 3

Napepld: N-acyl phosphatidylethanolamine phospholipase D

Nasp: nuclear autoantigenic sperm protein (histonebinding)

Ncam1: neural cell adhesion molecule 1

Ncam2: neural cell adhesion molecule 2

Ncdn: neurochondrin

Ncl: nucleolin

Ncstn: nicastrin

Ndel1: nudE nuclear distribution gene E homolog (A. nidulans)-like 1

Ndufa9: NADH dehydrogenase (ubiquinone) 1 alpha subcomplex, 9, $39 \mathrm{kDa}$

Ndufc2: NADH dehydrogenase (ubiquinone) 1, subcomplex unknown, $2,14.5 \mathrm{kDa}$

Ndufs1: NADH dehydrogenase (ubiquinone) Fe-S protein 1, $75 \mathrm{kDa}$ (NADH-coenzyme Q reductase)

Ndufs6: NADH dehydrogenase (ubiquinone) Fe-S protein $6,13 \mathrm{kDa}$ (NADH-coenzyme Q reductase)

Ndufs7: NADH dehydrogenase (ubiquinone) Fe-S protein 7, $20 \mathrm{kDa}$ (NADH-coenzyme Q reductase)

Nedd4: neural precursor cell expressed, developmentally down-regulated 4

Nef3: neurofilament, medium polypeptide

Nefh: neurofilament, heavy polypeptide

Negr1: neuronal growth regulator 1

Nell1: NEL-like 1 (chicken)

Neo1: neogenin homolog 1 (chicken)

Nes: nestin

Nexn: nexilin (F actin binding protein)
Nf2: neurofibromin 2 (merlin)

Nfix: nuclear factor I/X (CCAAT-binding transcription factor)

Nfkb1: nuclear factor of kappa light polypeptide gene enhancer in B-cells 1

Ninj1: ninjurin 1

Nlgn2: neuroligin 2

Nlgn3: neuroligin 3

Nme2: non-metastatic cells 2, protein (NM23B) expressed in

Nolc1: nucleolar and coiled-body phosphoprotein 1

Nos1: nitric oxide synthase 1 (neuronal)

Nos3: nitric oxide synthase 3 (endothelial cell)

Notch1: Notch homolog 1, translocation-associated (Drosophila)

Notch2: Notch homolog 2 (Drosophila)

Notch4: Notch homolog 4 (Drosophila)

Npc2: Niemann-Pick disease, type C2

Npdc1: neural proliferation, differentiation and control, 1

Npepps: aminopeptidase puromycin sensitive

Npm1: nucleophosmin (nucleolar phosphoprotein B23, numatrin)

Npr2: natriuretic peptide receptor B/guanylate cyclase B (atrionatriuretic peptide receptor B)

Npvf: neuropeptide VF precursor

Npy5r: neuropeptide Y receptor Y5

Nr1i2: nuclear receptor subfamily 1, group I, member 2

$\mathrm{Nr} 5 \mathrm{a} 2$ : nuclear receptor subfamily 5 , group A, member 2

Nr6a1: nuclear receptor subfamily 6, group A, member 1

Nras: neuroblastoma RAS viral (v-ras) oncogene homolog

Nrbf2: nuclear receptor binding factor 2

Nrp2: neuropilin 2

Nsf: N-ethylmaleimide-sensitive factor

Ntrk2: neurotrophic tyrosine kinase, receptor, type 2

Nucb1: nucleobindin 1

Nucb2: nucleobindin 2

Nudc: nuclear distribution gene C homolog (A. nidulans)

Nup88: nucleoporin $88 \mathrm{kDa}$

Oas3: $2^{\prime}-5^{\prime}$-oligoadenylate synthetase $3,100 \mathrm{kDa}$

Obscn: obscurin, cytoskeletal calmodulin and titininteracting RhoGEF 
Ociad1: OCIA domain containing 1

Odc1: ornithine decarboxylase 1

Odf2: outer dense fiber of sperm tails 2

Ogfr: opioid growth factor receptor

Olfm2: olfactomedin 2

Olfm3: olfactomedin 3

Omg: oligodendrocyte myelin glycoprotein

Optn: optineurin

Osbp: oxysterol binding protein

Oxr1: oxidation resistance 1

P4hb: prolyl 4-hydroxylase, beta polypeptide

Pabpc1: poly(A) binding protein, cytoplasmic 1

Pabpc4: poly(A) binding protein, cytoplasmic 4 (inducible form)

Padi2: peptidyl arginine deiminase, type II

Pak3: p21 protein (Cdc42/Rac)-activated kinase 3

Palm: paralemmin

Panx1: pannexin 1

Pard3: par-3 partitioning defective 3 homolog (C. elegans)

Parg: poly (ADP-ribose) glycohydrolase

Park7: Parkinson disease (autosomal recessive, early onset) 7

Pbp: phosphatidylethanolamine binding protein 1

Pcca: propionyl Coenzyme A carboxylase, alpha polypeptide

Pcdh21: protocadherin 21

Pcdha3: protocadherin alpha 3

Pcdhb1: protocadherin beta 1

Pcdhb10: protocadherin beta 10

Pcdhga12: protocadherin gamma subfamily A, 12

Pclo: piccolo (presynaptic cytomatrix protein)

Pcolce: procollagen C-endopeptidase enhancer

Pcsk1n: proprotein convertase subtilisin/kexin type 1 inhibitor

Pcsk5: proprotein convertase subtilisin/kexin type 5

Pcyox1: prenylcysteine oxidase 1

Pdap1: PDGFA associated protein 1

Pdcd4: programmed cell death 4 (neoplastic transformation inhibitor)

Pdcl: phosducin-like

Pde10a: phosphodiesterase 10A

Pde4b: phosphodiesterase 4B, cAMP-specific (phosphodiesterase E4 dunce homolog, Drosophila)

Pdlim7: PDZ and LIM domain 7 (enigma)

Pea15: phosphoprotein enriched in astrocytes 15
Pecr: peroxisomal trans-2-enoyl-CoA reductase

Per1: period homolog 1 (Drosophila)

Per3: period homolog 3 (Drosophila)

Pfkl: phosphofructokinase, liver

PGAP1: post-GPI attachment to proteins 1

Phactr3: phosphatase and actin regulator 3

Phb: prohibitin

Phka1: phosphorylase kinase, alpha 1 (muscle)

Phkg2: phosphorylase kinase, gamma 2 (testis)

Pi4ka: phosphatidylinositol 4-kinase, catalytic, alpha

Picalm: phosphatidylinositol binding clathrin assembly protein

Pik3c3: phosphoinositide-3-kinase, class 3

Pik3cb: phosphoinositide-3-kinase, catalytic, beta polypeptide

Pik3r2: phosphoinositide-3-kinase, regulatory subunit 2 (beta)

Pim1: pim-1 oncogene

Pip5k2a: phosphatidylinositol-5-phosphate 4-kinase, type II, alpha

Pitpnm1: phosphatidylinositol transfer protein, membrane-associated 1

Pkia: protein kinase (cAMP-dependent, catalytic) inhibitor alpha

Pkm2: pyruvate kinase, muscle

Pla2g2a: phospholipase A2, group IIA (platelets, synovial fluid)

Plb1: phospholipase B1

Plcb4: phospholipase $\mathrm{C}$, beta 4

Plcd4: phospholipase C, delta 4

Plec1: plectin 1, intermediate filament binding protein $500 \mathrm{kDa}$

Plk1: polo-like kinase 1 (Drosophila)

Pnkp: polynucleotide kinase 3'-phosphatase

Pnma1: paraneoplastic antigen MA1

Ppara: peroxisome proliferator-activated receptor alpha

Ppargclb: peroxisome proliferator-activated receptor gamma, coactivator 1 beta

ppfibp2: PTPRF interacting protein, binding protein 2 (liprin beta 2)

Ppia: peptidylprolyl isomerase A (cyclophilin A)

Ppm2c: pyruvate dehydrogenase phosphatase catalytic subunit 1

Ppp1r14b: protein phosphatase 1, regulatory (inhibitor) subunit $14 \mathrm{~B}$

Ppplrla: protein phosphatase 1, regulatory (inhibitor) subunit $1 \mathrm{~A}$ 
Ppp1r9a: protein phosphatase 1, regulatory (inhibitor) subunit $9 \mathrm{~A}$

Ppp2r1a: protein phosphatase 2 (formerly 2A), regulatory subunit $\mathrm{A}$, alpha isoform

Ppp3cc: protein phosphatase 3 (formerly $2 \mathrm{~B}$ ), catalytic subunit, gamma isoform

Pqlc1: PQ loop repeat containing 1

Prdx1: peroxiredoxin 1

Prdx6: peroxiredoxin 6

Prg3: proteoglycan 3

Prkaa2: protein kinase, AMP-activated, alpha 2 catalytic subunit

Prkaca: protein kinase, cAMP-dependent, catalytic, alpha

Prkar1a: protein kinase, cAMP-dependent, regulatory, type I, alpha (tissue specific extinguisher 1)

Prkar2a: protein kinase, cAMP-dependent, regulatory, type II, alpha

Prkcd: protein kinase C, delta

PRKCQ: protein kinase $\mathrm{C}$, theta

Prkwnk1: WNK lysine deficient protein kinase 1

Prlh: prolactin releasing hormone

Prmt3: protein arginine methyltransferase 3

Prpsap2: phosphoribosyl pyrophosphate synthetaseassociated protein 2

Prrx2: paired related homeobox 2

Prss12: protease, serine, 12 (neurotrypsin, motopsin)

Prss15: lon peptidase 1, mitochondrial

Prx: periaxin

Psap: prosaposin

Pscd2: cytohesin 2

Pscd3: cytohesin 3

Psg4: pregnancy specific beta-1-glycoprotein 4

Psma1: proteasome (prosome, macropain) subunit, alpha type, 1

Psma2: proteasome (prosome, macropain) subunit, alpha type, 2

Psmc4: proteasome (prosome, macropain) 26S subunit, ATPase, 4

Psme2: proteasome (prosome, macropain) activator subunit 2 (PA28 beta)

Ptbp1: polypyrimidine tract binding protein 1

Ptch1: patched homolog 1 (Drosophila)

Pth: parathyroid hormone

Pthr2: parathyroid hormone 2 receptor

Ptk2b: PTK2B protein tyrosine kinase 2 beta
Ptp4a1: protein tyrosine phosphatase type IVA, member 1

Ptpn11: protein tyrosine phosphatase, non-receptor type 11

Ptpn23: protein tyrosine phosphatase, non-receptor type 23

Ptprj: protein tyrosine phosphatase, receptor type, J

Ptprr: protein tyrosine phosphatase, receptor type, $\mathrm{R}$

Ptprv: protein tyrosine phosphatase, receptor type, $\mathrm{V}$ (pseudogene)

Pxn: paxillin

Pygl: phosphorylase, glycogen, liver

Qscn6: quiescin Q6 sulfhydryl oxidase 1

Rab10: RAB10, member RAS oncogene family

Rab14: RAB14, member RAS oncogene family

Rab2: RAB2A, member RAS oncogene family

Rab21: RAB21, member RAS oncogene family

Rab35: RAB35, member RAS oncogene family

Rab3il1: RAB3A interacting protein (rabin3)-like 1

Rab4a: RAB4A, member RAS oncogene family

Rab5a: RAB5A, member RAS oncogene family

Rab7: RAB7A, member RAS oncogene family

Rab9: RAB9A, member RAS oncogene family

Rabac1: Rab acceptor 1 (prenylated)

Rabggta: Rab geranylgeranyltransferase, alpha subunit

Rac1: ras-related C3 botulinum toxin substrate 1 (rho family, small GTP binding protein Racl)

Rala: v-ral simian leukemia viral oncogene homolog A (ras related)

RalB: v-ral simian leukemia viral oncogene homolog B (ras related; GTP binding protein)

Ralb: v-ral simian leukemia viral oncogene homolog B (ras related; GTP binding protein)

Raly: RNA binding protein, autoantigenic (hnRNP associated with lethal yellow homolog (mouse))

RanGap1: Ran GTPase activating protein 1

Rap2b: RAP2B, member of RAS oncogene family

Rbbp6: retinoblastoma binding protein 6

Rbl2: retinoblastoma-like 2 (p130)

Rbm3: RNA binding motif (RNP1, RRM) protein 3

Rcn1: reticulocalbin 1, EF-hand calcium binding domain

Rcvrn: recoverin

Rdh10: retinol dehydrogenase 10 (all-trans)

Rdx: radixin

Rest: RE1-silencing transcription factor 
Rgs4: regulator of G-protein signaling 4

Rgs5: regulator of G-protein signaling 5

Rgs7: regulator of G-protein signaling 7

Rhoa: ras homolog gene family, member A

RhoB: ras homolog gene family, member B

Rhoj: ras homolog gene family, member J

Rims1: regulating synaptic membrane exocytosis 1

Rnasel: ribonuclease L ( $2^{\prime}, 5^{\prime}$-oligoisoadenylate synthetase-dependent)

Rnf36: tripartite motif-containing 69

Rnf40: ring finger protein 40

Robo1: roundabout, axon guidance receptor, homo$\log 1$ (Drosophila)

Robo4: roundabout homolog 4, magic roundabout (Drosophila)

Rock1: Rho-associated, coiled-coil containing protein kinase 1

Rock2: Rho-associated, coiled-coil containing protein kinase 2

rora: RAR-related orphan receptor A

Rpe65: retinal pigment epithelium-specific protein $65 \mathrm{kDa}$

Rpl13: ribosomal protein L13

Rpl6: ribosomal protein L6

Rplp1: ribosomal protein, large, $\mathrm{P} 1$

Rpn2: ribophorin II

Rps15: ribosomal protein S15

Rps16: ribosomal protein S16

Rps6ka2: ribosomal protein S6 kinase, $90 \mathrm{kDa}$, polypeptide 2

Rtcd1: RNA terminal phosphate cyclase domain 1

Rtkn: rhotekin

Rtn1: reticulon 1

Rtn3: reticulon 3

Rtn4: reticulon 4

Rtn4r: reticulon 4 receptor

Samsn1: SAM domain, SH3 domain and nuclear localization signals 1

Sardh: sarcosine dehydrogenase

Sart1: squamous cell carcinoma antigen recognized by $\mathrm{T}$ cells

Sbds: Shwachman-Bodian-Diamond syndrome

Sc4mol: sterol-C4-methyl oxidase-like

Sca10: ataxin 10

Scg2: secretogranin II (chromogranin C)

Scg3: secretogranin III
Scn1a: sodium channel, voltage-gated, type I, alpha subunit

Scn2a1: sodium channel, voltage-gated, type II, alpha subunit

Scn5a: sodium channel, voltage-gated, type V, alpha subunit

Scn8a: sodium channel, voltage gated, type VIII, alpha subunit

Scoc: short coiled-coil protein

Scp2: sterol carrier protein 2

SCYB11: chemokine (C-X-C motif) ligand 11

Scye1: aminoacyl tRNA synthetase complexinteracting multifunctional protein 1

Sdfr1: neuroplastin

Sdpr: serum deprivation response

SH3gl1: SH3-domain GRB2-like 1

SH3glb1: SH3-domain GRB2-like endophilin B1

Shank1: SH3 and multiple ankyrin repeat domains 1

Sirpa: signal-regulatory protein alpha

Skiv212: superkiller viralicidic activity 2-like 2 (S. cerevisiae)

Slc12a2: solute carrier family 12 (sodium/potassium/chloride transporters), member 2

Slc12a9: solute carrier family 12 (potassium/chloride transporters), member 9

Slc13a3: solute carrier family 13 (sodium-dependent dicarboxylate transporter), member 3

Slc17a6: solute carrier family 17 (sodium-dependent inorganic phosphate cotransporter), member 6

Slcla1: solute carrier family 1 (neuronal/epithelial high affinity glutamate transporter, system Xag), member 1

Slc26a4: solute carrier family 26, member 4

Slc27a5: solute carrier family 27 (fatty acid transporter), member 5

Slc30a1: solute carrier family 30 (zinc transporter), member 1

Slc44a4: solute carrier family 44, member 4

Slc5a1: solute carrier family 5 (sodium/glucose cotransporter), member 1

Slc6a8: solute carrier family 6 (neurotransmitter transporter, creatine), member 8

Slc8a1: solute carrier family 8 (sodium/calcium exchanger), member 1

Smad4: SMAD family member 4

Smc1l1: structural maintenance of chromosomes $1 \mathrm{~A}$ Smoc1: SPARC related modular calcium binding 1

Snai2: snail homolog 2 (Drosophila) 
Snap25: synaptosomal-associated protein, $25 \mathrm{kDa}$

Snap91: synaptosomal-associated protein, $91 \mathrm{kDa}$ homolog (mouse)

Snca: synuclein, alpha (non A4 component of amyloid precursor)

Sncb: synuclein, beta

Snx3: sorting nexin 3

Snx7: sorting nexin 7

sod1: superoxide dismutase 1 , soluble

Sod2: superoxide dismutase 2, mitochondrial

Son: SON DNA binding protein

Sord: sorbitol dehydrogenase

Sox5: SRY (sex determining region Y)-box 5

Sp4: Sp4 transcription factor

Sp7: Sp7 transcription factor

Sqle: squalene epoxidase

Srfbp1: serum response factor binding protein 1

Sst: somatostatin

St6gal2: ST6 beta-galactosamide alpha-2,6-sialyltransferase 2

St7l: suppression of tumorigenicity 7 like

Stard3nl: STARD3 N-terminal like

Stat3: signal transducer and activator of transcription 3 (acute-phase response factor)

Stat5a: signal transducer and activator of transcription $5 \mathrm{~A}$

Stip1: stress-induced-phosphoprotein 1

Stk2: NIMA (never in mitosis gene a)-related kinase 4

Stk22a: testis-specific serine kinase 1A pseudogene

Stmn1: stathmin 1

Stmn2: stathmin-like 2

Stmn4: stathmin-like 4

Strbp: spermatid perinuclear RNA binding protein

Stx1a: syntaxin 1A (brain)

Stx1b2: syntaxin 1B

Stx5: syntaxin 5

stxbp1: syntaxin binding protein 1

Stxbp3: syntaxin binding protein 3

Sulf1: sulfatase 1

Svop: SV2 related protein homolog (rat)

SYAP1: synapse associated protein 1, SAP47 homolog (Drosophila)

Sycp1: synaptonemal complex protein 1

Sycp2: synaptonemal complex protein 2

Synj2: synaptojanin 2
Syt3: synaptotagmin III

Syt4: synaptotagmin IV

Tacc2: transforming, acidic coiled-coil containing protein 2

Tacc3: transforming, acidic coiled-coil containing protein 3

Tbx2: T-box 2

Tceb3: transcription elongation factor B (SIII), polypeptide $3(110 \mathrm{kDa}$, elongin $\mathrm{A})$

Tcp1: t-complex 1

Tcp11: t-complex 11 homolog (mouse)

Tdg: thymine-DNA glycosylase

Tdrd7: tudor domain containing 7

Tesk2: testis-specific kinase 2

Tfrc: transferrin receptor (p90, CD71)

Tgfb1: transforming growth factor, beta 1

Thrap3: thyroid hormone receptor associated protein 3

Thrb: thyroid hormone receptor, beta (erythroblastic leukemia viral (v-erb-a) oncogene homolog 2, avian)

Thy1: Thy-1 cell surface antigen

Tinag: tubulointerstitial nephritis antigen

Tkt: transketolase

Tle3: transducin-like enhancer of split 3 (E(sp1) homolog, Drosophila)

Tlr5: toll-like receptor 5

Tm4sf8: tetraspanin 3

Tm9sf2: transmembrane 9 superfamily member 2

Tmeff1: transmembrane protein with EGF-like and two follistatin-like domains 1

Tmem17: transmembrane protein 17

Tmlhe: trimethyllysine hydroxylase, epsilon

Tmod2: tropomodulin 2 (neuronal)

Tmpo: thymopoietin

Tnfrsf14: tumor necrosis factor receptor superfamily, member 14 (herpesvirus entry mediator)

Top1: topoisomerase (DNA) I

Tpd52l2: tumor protein D52-like 2

Tpm4: tropomyosin 4

Tra1: heat shock protein 90kDa beta (Grp94), member 1

Traf4: TNF receptor-associated factor 4

Trib3: tribbles homolog 3 (Drosophila)

Trim10: tripartite motif-containing 10

Trim25: tripartite motif-containing 25

Trim50: tripartite motif-containing 50 
Trpal: transient receptor potential cation channel, subfamily A, member 1

TrpV6: transient receptor potential cation channel, subfamily $\mathrm{V}$, member 6

Tsc1: tuberous sclerosis 1

Tsga 10: testis specific, 10

Tshr: thyroid stimulating hormone receptor

Ttc1: tetratricopeptide repeat domain 1

Ttn: titin

Tub: tubby homolog (mouse)

Tubb: tubulin, beta

Tubb2: tubulin, beta $2 \mathrm{~A}$

Txn: thioredoxin

U2af2: U2 small nuclear RNA auxiliary factor 2

Ubc: ubiquitin C

Ube213: ubiquitin-conjugating enzyme E2L 3

Ubqln1: ubiquilin 1

Uchl1: ubiquitin carboxyl-terminal esterase L1 (ubiquitin thiolesterase)

Ugcgll: UDP-glucose glycoprotein glucosyltransferase 1

Uhrf1: ubiquitin-like with PHD and ring finger domains 1

Unc13: unc-13 homolog B (C. elegans)

Unc13a: unc-13 homolog A (C. elegans)

Unc13d: unc-13 homolog D (C. elegans)

Uqcrfs1: ubiquinol-cytochrome c reductase, Rieske iron-sulfur polypeptide 1

Usp14: ubiquitin specific peptidase 14 (tRNAguanine transglycosylase)

Usp15: ubiquitin specific peptidase 15

Vamp2: vesicle-associated membrane protein 2 (synaptobrevin 2)

Vang12: vang-like 2 (van gogh, Drosophila)

Vapa: VAMP (vesicle-associated membrane protein)associated protein $\mathrm{A}, 33 \mathrm{kDa}$

Vapb: VAMP (vesicle-associated membrane protein)associated protein $\mathrm{B}$ and $\mathrm{C}$

Vav1: vav 1 guanine nucleotide exchange factor

Vcp: valosin-containing protein

Vdac2: voltage-dependent anion channel 2

Vegfa: vascular endothelial growth factor A

Vgf: VGF nerve growth factor inducible

Viaat: solute carrier family 32 (GABA vesicular transporter), member 1

Vim: vimentin

Vldlr: very low density lipoprotein receptor
Vps4a: vacuolar protein sorting 4 homolog A (S. cerevisiae)

Vps52: vacuolar protein sorting 52 homolog (S. cerevisiae)

Vps54: vacuolar protein sorting 54 homolog (S. cerevisiae)

Vsnll: visinin-like 1

Vtila: vesicle transport through interaction with tSNAREs homolog 1A (yeast)

Vtn: vitronectin

Wbp11: WW domain binding protein 11

Wbscr1: eukaryotic translation initiation factor $4 \mathrm{H}$

Whsc2: Wolf-Hirschhorn syndrome candidate 2

Wif1: WNT inhibitory factor 1

Wnk4: WNK lysine deficient protein kinase 4

Wrnip1: Werner helicase interacting protein 1

Xpo7: exportin 7

Xrcc5: X-ray repair complementing defective repair in Chinese hamster cells 5

Ybxl: Y box binding protein 1

yes1: v-yes-1 Yamaguchi sarcoma viral oncogene homolog 1

Ywhah: tyrosine 3-monooxygenase/tryptophan 5monooxygenase activation protein, eta polypeptide

Ywhaq: tyrosine 3-monooxygenase/tryptophan 5monooxygenase activation protein, theta polypeptide

Zbtb7a: zinc finger and BTB domain containing 7A

Zeb1: zinc finger E-box binding homeobox 1

Zfhx2: zinc finger homeobox 2

Zfp57: zinc finger protein 57 homolog (mouse)

Zmynd19: zinc finger, MYND-type containing 19

Znf219: zinc finger protein 219

Znf291: S-phase cyclin A-associated protein in the ER

Znf292: zinc finger protein 292

Znf382: zinc finger protein 382.

\section{C.}

Cellular signaling pathway clusters of extracted proteins from primary cortical tissue. Specific cellular signaling pathway clusters generated in an un-biased manner using Ingenuity Pathway Analysis (v. 8.5). The relative score generated for the degree of pathway population by proteins from the respective input sets (control or $3 \times \mathrm{TgAD}$ ) is shown in bold. The following are different types of signaling pathways.

C.1. ERK/MAPK Signaling (Enrichment Ratio* $-\log 10(p)$ ). 
Control Protein (1.32502). PPP2R2A, CRK, PPP1R14B, DUSP2, PTK2, SHC1, PAK1, PPP1R10, PPP1R7, PIK3CG, STAT1, PRKCA, ETS1, SRC, PAK2, YWHAB, CRKL, PRKAR2A, STAT3, PLA2G4C, FOS, PPP2CB, PPP2R1A, PRKAR2B, PRKCI, PAK3, PPP2R2B, PRKACA, PPP2R5E, ELK1, PPP2R1B, PRKAR1A, PRKCB.

3xTgAD Protein (0.1537). PXN, YWHAH, PTK2B, RAC1, PRKAR2A, PLA2G2A, STAT3, MYC, YWHAQ (includes EG:10971), PPP2R1A, PAK3, PIK3C3, PRKCD, PRKACA, PIK3CB, PIK3R2, ELK1, FYN, PRKAR1A.

C.2. Inositol Phosphate Metabolism (Enrichment Ratio* $-\log 10(p))$.

Control Protein (0.86424). PDIA3, OCRL, PAK1, PIK3CG, PRKAA2, PLCB1, PLCL1, PI4KA, PMPCA, ATM, GRK4, PRKCQ, PAK2, CDK7, CDK6, PLK1, GRK5, ITPKA, SYNJ2, PIP5K1A, PAK3, SYNJ1, PIP5K1C, GRK6, PIP4K2A, PIP4K2C, CDK2.

$3 x T g A D$ Protein (0.15355). PLK1, INPP5D, SYNJ2, INPP4A, INPP4B, INPP4B, PAK3, PIM1, PRKCD, PIK3C3, PRKAA2, PIK3CB, PIK3R2, PIP4K2A, PLCD4, PI4KA, ATM.

C.3. Wnt/ $\beta$-Catenin Signaling (Enrichment Ratio* $-\log 10(p))$.

Control Protein (0.5609). PPP2R2A, SOX10, CSNK1E, WIF1, WNT7A, TGFB1, SMO, CSNK2A1, CSNK2B, CTNNB1, SRC, SFRP4, GJA1, CSNK1G2, DVL1, FZD9, PPP2CB, CDH2, CDH1, PPP2R1A, PPP2R2B, TLE3, PPP2R5E, UBC, PPP2R1B, WNT5A.

3xTgAD Protein (0.12772). CSNK1G1, CSNK1G3, DVL1, ACVR1, APC, MYC, CSNK1E, PPP2R1A, CDH2, CDH1, WIF1, TGFB1, GNAO1, TLE3, UBC, CTNNB1, SOX5 (includes EG:6660).

C.4. Calcium Signaling (Enrichment Ratio* $-\log 10(p)$ ).

Control Protein (0.33825). TRPC2, GRIN2A, TNNI2, GRIA1, RCAN2, CALM2, CHRNB4, TRPC5, TNNT3, RYR3, GRIK1, GRIN2B, PRKAR2A, TRPC4, PPP3CC, TRPC7, CHRNG, PRKAR2B, TPM3, CAMKK1, PRKACA, TNNI1, CHRNB3, CAMKK2, PRKAR1A.

3xTgAD Protein (0.110875). RAP2B, MYH10, CALR, MYH6, ATP2B1, PRKAR2A, TPM4, PPP3CC, ATP2B2, HDAC6, TRPV6, HTR3A, CAMKK1, PRKACA, MYH9, SLC8A1, ACTA1, PRKAR1A.

C.5. Tight Junction Signaling (Enrichment Ratio* $-\log 10(p)$ ).

Control Protein (0.4147). TJP2, CDC42, TJP1, PPP2R2A, CLDN19, VAPA, PRKAR2A, PRKCZ, FOS, PPP2CB,
PPP2R1A, PRKCI, PRKAR2B, CLDN4, CLDN1, TGFB1, PPP2R2B, PRKACA, PPP2R5E, STX4, CTNNB1, PPP2R1B, PRKAR1A.

$3 x T g A D$ Protein (0.24514). MYH10, MYH6, CDC42, ACTB, HSF1, CLDN18, VAPA, RAC1, PRKAR2A, MPDZ, PPP2R1A, TGFB1, RHOA, CEBPA, PRKACA, MYH9, CTNNB1, ACTA1, PRKAR1A.

C.6. NF- $\kappa$ B Signaling (Enrichment Ratio* $-\log 10(p)$ ).

Control Protein (0.12096). PRKCQ, EGF, PRKCZ, TLR9, TLR4, TLR5, PIK3CG, TLR6, PRKACA, CSNK2A1, PDGFRA, TLR3, CSNK2B, PRKCB, EGFR.

3xTgAD Protein (0.0190128). IL1R2, TLR5, BCL10, PIK3C3, PRKACA, IL1B, PIK3CB, PIK3R2, NFKB1, EGFR.

C.7. PTEN Signaling (Enrichment Ratio* $-\log 10(p))$.

Control Protein (0.1368). PTK2, SHC1, CDC42, PIK3CG, CSNK2A1, PDGFRA, CSNK2B, CDKN1B, PRKCZ, FASLG, EGFR.

$3 x T g A D$ Protein (0.1199). CDC42, YWHAH, RAC1, PIK3CB, PIK3R2, CDKN1B, NFKB1, INPP5D, EGFR, MAGI3.

C.8. SAPK/JNK Signaling (Enrichment Ratio* $-\log 10(p)$ ).

Control Protein (0.0544984). SHC1, CDC42, GADD45A, CRKL, PIK3CG, CRK, ELK1, GNG7.

$3 x T g A D$ Protein (0.0717288). CDC42, GADD45A, IRS1, PIK3C3, RAC1, PIK3CB, PIK3R2, ELK1, HNRNPK.

C.9. PI3K/AKT Signaling (Enrichment Ratio* $-\log 10(p)$ ).

Control Protein (0.15481). TSC1, PPP2R2A, YWHAB, PRKCZ, PPP2CB, SHC1, PPP2R1A, PIK3CG, TSC2, PPP2R2B, PPP2R5E, CDKN1B, CTNNB1, PPP2R1B.

3xTgAD Protein (0.19323). TSC1, JAK1, YWHAH, JAK2, NOS3, INPP5D, YWHAQ (includes EG:10971), MTOR, PPP2R1A, HSP90AB1, PIK3CB, PIK3R2, CDKN1B, CTNNB1.

C.10. p38 MAPK Signaling (Enrichment Ratio* $-\log 10(p)$ ).

Control Protein (0).

$3 x T g A D$ Protein (0.0630702). IL1R2, MYC, TGFB1, MAP3K7IP2, IL1B, PLA2G2A, EEF2K, MAPKAPK2, ELK1. 


\section{C.11. p53 Signaling (Enrichment Ratio* $-\log 10(p)$ ).}

Control Protein (0.037996). GADD45A, FASN, PIK3CG, SNAI2, CTNNB1, CDK2, ATM, SERPINE2.

3xTgAD Protein (0.12075). CCND2, GADD45A, PIK3C3, SNAI2, CABC1, PIK3CB, PIK3R2, BAX, CTNNB1, ATM.

C.12. JAK/Stat Signaling (Enrichment Ratio* $-\log 10(p))$.

Control Protein (0.051714). SHC1, SOCS1, PTPN11, PIK3CG, STAT3, STAT1.

3xTgAD Protein (0.25857). STAT5A, MTOR, JAK1, PTPN11, PIK3C3, PIK3CB, STAT3, PIK3R2, JAK2.

D.

Neuronal function pathway clusters of extracted proteins from primary cortical tissue. Specific neuronal function pathway clusters generated in an un-biased manner using Ingenuity Pathway Analysis (v. 8.5). The relative score generated for the degree of pathway population by proteins from the respective input sets (control or $3 \times T$ TgD) is shown in bold. The following are different types of signaling pathways.

D.1. Synaptic Long-Term Potentiation (Enrichment Ratio* $-\log 10(p))$.

Control Protein (1.27872). GRIN2B, GRIN2A, PRKCQ, PPP1R1A, GRIA1, PRKAR2A, CALM2, CACNA1C, PPP3CC, GRM4, PPP1R14B, PRKCZ, GRM5, PRKCI, PRKAR2B, PPP1R10, PPP1R7, PPP1R14D, PRKACA, PLCB1, PRKD1, PRKCA, PRKAR1A, PRKCB

$3 x T g A D$ Protein (0).

D.2. Axonal Guidance Signaling (Enrichment Ratio* $-\log 10(p))$.

Control Protein (0.69525). NOS1, GUCY1B2, PRKCQ, GUCY1A3, GUCY2D, PPP2R2A, GRID2, GRIA1, GRM4, PRKCZ, GRM5, PPP2CB, PPP2R1A, PRKCI, PPP2R2B, RYR3, GUCY1A2, PLCB1, PPP2R5E, PPP2R1B, PRKD1, PRKCA, PRKCB.

$3 x T g A D$ Protein (0.1918). NOS1, GUCY2D, GNAI1, PLA2G2A, GNAZ, NOS3, PRDX6, GNAI2, PPP2R1A, PRKCD, GNAO1, IGF1R, GUCY2F, ADCY8, NPR2.

D.3. Synaptic Long-Term Depression (Enrichment Ratio* $-\log 10(p))$.

Control Protein (0.47428). NOS1, GUCY1B2, PRKCQ, GUCY1A3, GUCY2D, PPP2R2A, GRID2, GRIA1, GRM4,
PRKCZ, GRM5, PPP2CB, PPP2R1A, PRKCI, PPP2R2B, RYR3, GUCY1A2, PLCB1, PPP2R5E, PPP2R1B, PRKD1, PRKCA, PRKCB.

$3 x T g A D$ Protein (0.11112). NOS1, GUCY2D, GNAI1, PLA2G2A, GNAZ, NOS3, PRDX6, GNAI2, PPP2R1A, PRKCD, GNAO1, IGF1R, GUCY2F, ADCY8, NPR2.

D.4. Parkinson's Signaling (Enrichment Ratio* $-\log 10(p)$ ).

Control Protein (0.168256). UCHL1, PARK7, SNCA.

$3 x T g A D$ Protein (0.18656). UCHL1, PARK7, SNCA.

D.5. Huntington's Disease Signaling (Enrichment Ratio* $-\log 10(p))$.

Control Protein (0.20048). EGF, GNG7, PRKCZ, TGM2, CTSD, SHC1, PIK3CG, VAMP3, PLCB1, PRKD1, PRKCA, EGFR, SDHA, GRIN2B, PRKCQ, YKT6, SH3GL3, STX1A, SNAP25, TAF9B, GRM5, PRKCI, UBC, GOSR2, SNCA, PRKCB.

$3 x T g A D$ Protein (0.36179). VTI1A, HSPA14, REST, GNB2L1, HDAC6, NSF, MTOR, PIK3C3, IGF1R, PIK3R2, EGFR, CAPN5, CAPN6, CLTC, BAX, STX1A, SNAP25, DNM1, DNAJC5, ATP5B, CAPN1, PRKCD, HAP1, DCTN1, PIK3CB, UBC, SNCA, CASP7.

D.6. Regulation of Actin-Based Motility by Rho (Enrichment Ratio* $\left.^{*} \log 10(p)\right)$.

Control Protein (0.1476). PIP5K1A, WIPF1, PAK1, PAK2, CDC42, CFL1, PAK3, PIP5K1C, PIP4K2A, PIP4K2C, PI4KA.

$3 x T g A D$ Protein (0.3243). CDC42, CFL1, ACTB, RAC1, RHOJ, ROCK1, PAK3, RHOB, RHOA, ARHGDIA, PIP4K2A, ACTA1, PI4KA.

D.7. Amyotrophic Lateral Sclerosis Signaling (Enrichment Ratio* $\left.^{*} \log 10(p)\right)$.

Control Protein (0.23184). NOS1, GRIN2B, GRIN2A, SOD1, GRIA1, PGF, VEGFA, PAK1, GRIK4, PIK3CG, CAT, GLUL, SSR4.

$3 x T g A D$ Protein $(\boldsymbol{0} .55955)$. NOS1, CAPN5, CAPN6, SOD1, RAB5A, RAC1, NEFH, BAX, CCS, VEGFA, CAPN1, PIK3C3, NEFM, PIK3CB, PIK3R2, CASP7.

D.8. Actin Cytoskeleton Signaling (Enrichment Ratio* $-\log 10(p))$.

Control Protein (0.0763861). PAK2, CFL1, CDC42, CRKL, EGF, CRK, TTN, PTK2, SHC1, PAK1, PIP5K1A, PAK3, 
PIP5K1C, FGF18, PIK3CG, EZR, FGF23, PIP4K2A, PIP4K2C, GIT1.

3xTgAD Protein (0.44928). MYH10, MYH6, CDC42, ROCK2, PIK3C3, EZR, PIK3R2, LBP, ACTA1, PXN, ARHGEF12, CFL1, ACTB, RAC1, RDX, APC, TTN, ROCK1, PAK3, RHOA, ARHGEF6, MYH9, VAV1, PIK3CB, ACTN4, PIP4K2A, MSN.

D.9. Amyloid Processing (Enrichment Ratio* $-\log 10(p)$ ).

Control Protein (0.20636). CSNK1E, PRKAR2B, CSNK2A, PRKAR2A, PRKACA, NCSTN, CSNK2B, PRKAR1A.

3xTgAD Protein (0.66144). CSNK1E, CAPN5, CAPN6, CAPN1, MARK1, PRKAR2A, PRKACA, NCSTN, BACE1, APP, PRKAR1A.

\section{E.}

Energy regulation/Metabolism pathway clusters of extracted proteins from primary cortical tissue. Specific energy regulation/metabolism pathway clusters generated in an unbiased manner using Ingenuity Pathway Analysis (v. 8.5). The relative score generated for the degree of pathway population by proteins from the respective input sets (control or $3 \mathrm{xTgAD}$ ) is shown in bold. The following are different types of signaling pathways.

E.1. Amino Sugars Metabolism (Enrichment Ratio* $-\log 10(p))$.

Control Protein (0.61425). FMO3, PDE7A, PDE10A, GM2A, CYB5R1, PDE4A, PDE4B, PDE1A, PDE4D, GFPT2, PDE1C, PDE7B, GALK1, CYB5R3.

3xTgAD Protein (0.0471409). HK1, CYB5R4, PDE10A, GALK1, CYB5R3, PDE4B.

E.2. Pentose Phosphate Pathway (Enrichment Ratio* $-\log 10(p))$.

Control Protein (0.48608). GPI, PGD, TKT, TALDO1, PRPSAP1, PGM1, PRPSAP2, PFKP, PFKL, PDHB, PFKM.

3xTgAD Protein (0.0245154). GPI, TKT, PRPSAP2, PFKL.

E.3. Glutamate Metabolism (Enrichment Ratio* $-\log 10(p)$ ).

Control Protein (0.31165). FMO3, GPT, GLUL, GCLC, GLUD1, GOT1, GOT2, GFPT2, EPRS.
$3 x \operatorname{TgAD}$ Protein (0).

E.4. Pantothenate and CoA Biosynthesis (Enrichment Ratio* $-\log 10(p))$.

Control Protein (0.34188). ENPP3, CRMP1, BCAT1, DPYD, DPYSL3, ENPP5, ENPP2.

$3 x T g A D$ Protein (0.0348908). CRMP1, DPYD, ENPP2.

E.5. Glycolysis/Gluconeogenesis (Enrichment Ratio* $-\log 10(p))$.

Control Protein (0.28363). PKM2, PGK1, ENO3, ENO2, GAPDH (includes EG:2597), PGM1, ALDH1L1, PFKP, PFKL, PDHB, PFKM, GPI, GALK1, DLAT, PGAM1, DLD.

$3 x T g A D$ Protein (0.0610449). PKM2, HK1, ADH1A, GPI, ALDH1A3, DLAT, GALK1, ENO2, PFKL, ACSL1.

E.6. Citrate Cycle (Enrichment Ratio* $-\log 10(p)$ ).

Control Protein (0.1581). SDHA, PC, CS, SUCLG1, DLD, PCK1.

$3 x T g A D$ Protein (0).

E.7. Galactose Metabolism.

Control Protein (0.104958). GLA, GALT, GALK1, GAA, PGM1, PFKP, PFKL, PFKM.

3xTgAD Protein (0.0486688). HK1, GLA, GALK1, GAA, PFKL, AKR1B1.

E.8. Glycosphingolipid Biosynthesis-Globoseries (Enrichment Ratio $\left.^{*}-\log 10(p)\right)$.

Control Protein (0.0411384). GLA, ST3GAL1, GM2A.

$3 x \operatorname{TgAD}$ Protein (0).

E.9. Glycosphingolipid Biosynthesis-Ganglioseries (Enrichment Ratio* $-\log 10(p))$.

Control Protein (0.008832). ST3GAL1, GM2A.

$3 x T g A D$ Protein (0).

E.10. N-Glycan Biosynthesis (Enrichment Ratio* $-\log 10(p)$ ).

Control Protein (0).

$3 x T g A D$ Protein $(\mathbf{0 . 0 1 5 2 7 2})$. RPN2, ST6GAL2, MGAT5, MGAT1. 
E.11. Pentose and Glucuronate Interconversions (Enrichment Ratio* $\left.^{*} \log 10(p)\right)$.

Control Protein (0).

3xTgAD Protein (0.0184464). UCHL1, B3GAT1, HPSE, DCXR, AKR1B1.

E.12. Fatty Acid Elongation in Mitochondria (Enrichment Ratio $\left.^{*}-\log 10(p)\right)$.

Control Protein (0).

$3 x T g A D$ Protein (0.0206016). ECHS1, PECR.

E.13. Glycerophospholipid Metabolism (Enrichment Ratio* $-\log 10(p))$.

Control Protein (0.0232106). GPAM, PCYT1B, PLD3, CDS1, PDIA3, GNPAT, DGKB, DGKG, PLCB1, PLCL1.

$3 x T g A D$ Protein (0.048204). AGPAT4, CDS1, GNPAT, CHKA, AGPAT1, DGKG, PLA2G2A, CLCF1, PHKA1, PLCD4, PRDX6.

E.14. Fatty Acid Biosynthesis (Enrichment Ratio* $-\log 10(p))$.

Control Protein (0).

$3 x T g A D$ Protein (0.0329256). ACACB, ACACA.

E.15. Purine Metabolism (Enrichment Ratio* $-\log 10(p)$ ).

Control Protein (0.08616). ENTPD8, PDE7A, PDE4A, DNTT, PDE1A, PDE4D, PSMC5, PDE7B, VCP, DHX16, ENPP2, PRPSAP2, PKM2, TJP2, GUCY1B2, GUCY1A3, GUCY2D, PDE10A, ENPP5, PDE4B, PDE1C, ENPP3, PSMC1, ENTPD2, PRPSAP1, GUCY1A2, PDE5A, DDX1, GMPR2, PSMC3.

3xTgAD Protein (0.124932). MYH6, ENTPD8, NME2, DNTT, WRNIP1, ABCC1, VCP, KATNA1, ATP5H (includes EG:10476), ENPP2, PRPSAP2, ADCY8, ATP5F1, ATP5I, ATP5J, PKM2, ATP1B1, GUCY2D, PDE10A, AMPD1, ATP5A1, AK3, PSMC4, PDE4B, DDX19B, ATP5B, GUCY2F, MYH9, DDX1, NPR2.

E.16. Pyruvate Metabolism (Enrichment Ratio* $-\log 10(p)$ ).

Control Protein (0.0214935). PKM2, PC, DLAT, DLD, PCK1, PDHB, GLO1.

$3 x T g A D$ Protein (0.070173). PKM2, ACACB, ALDH1A3, DLAT, ACAT1, ACACA, MDH1, AKR1B1, ACSL1.
E.17. Butanoate Metabolism (Enrichment Ratio* $-\log 10(p))$.

Control Protein (0).

$3 x T g A D$ Protein (0.0523452). ECHS1, ALDH1A3, AACS, ACAT1, MYO5B, DCXR, HMGCS1.

E.18. Synthesis and Degradation of Ketone Bodies (Enrichment Ratio $\left.^{*}-\log 10(p)\right)$.

Control Protein (0).

3xTgAD Protein (0.071505). ACAT1, HMGCS1.

\section{F.}

Cellular stress/damage pathway clusters of extracted proteins from primary cortical tissue. Specific energy cellular stress/damage pathway clusters generated in an un-biased manner using Ingenuity Pathway Analysis (v. 8.5). The relative score generated for the degree of pathway population by proteins from the respective input sets (control or $3 \mathrm{xTgAD}$ ) is shown in bold. The following are different types of signaling pathways.

F.1. NRF2-mediated Oxidative Stress Response (Enrichment Ratio $\left.^{*}-\log 10(p)\right)$.

Control Protein (0.44064). PRDX1, PPIB, GCLC, PRKCZ, GSTM2, SOD2, PIK3CG, VCP, DNAJA2, TXN, FKBP5, PRKD1, CBR1, PRKCA, GSTK1, SOD1, PRKCQ, TXNRD1, GSTO1, FOS, PRKCI, ERP29, STIP1, CAT, PRKCB, EPHX1.

$3 x T g A D$ Protein (0.23751). USP14, SOD1, PRDX1, ACTB, GSTM3 (includes EG:2947), JUNB, DNAJC5, GSTM2, SOD2, ERP29, STIP1, PIK3C3, PRKCD, ABCC1, VCP, PIK3CB, FOSL1, PIK3R2, TXN, EIF2AK3, ACTA1.

F.2. Ceramide Signaling (Enrichment Ratio* $-\log 10(p))$.

Control Protein (0.15748). CTSD, PPP2CB, FOS, PPP2R1A, PPP2R2A, PIK3CG, PPP2R2B, PPP2R5E, PPP2R1B.

$3 x T g A D$ Protein (0).

F.3. Glutathione Metabolism (Enrichment Ratio* $-\log 10(p))$.

Control Protein (0.094285). OPLAH, PGD, GSTM2, GGT5, GCLC, GGT1, GSTO1, GSTK1, GGT7.

$3 x \operatorname{TgAD}$ Protein (0).

F.4. LPS/IL-1 Mediated Inhibition of RXR Function (Enrichment Ratio* $-\log 10(p))$. 
Control Protein (0.19071). SULT1C2, FMO3, CPT1A, CHST7, ALDH1L1, NR1H3, GSTO1, SULT4A1, GSTM2, NR1I2, SREBF1, SULT1A1, CAT, CPT2, FABP4, XPO1, FABP1, FABP7, SULT1E1, FABP3, GSTK1, TLR4, FABP2.

3xTgAD Protein (0.13872). PPARA, GSTM3 (includes EG:2947), ABCA1, IL1R2, ABCC2, SLC27A5, GSTM2, ALDH1A3, NR1I2, CPT2, ACSL4, FABP1, IL1B, NR5A2, CHST10, LBP, ABCC4, HMGCS1, ACSL1, MAOA.

F.5. Fatty Acid Elongation in Mitochondria (Enrichment Ratio* $\left.^{*} \log 10(p)\right)$.

Control Protein (0).

3xTgAD Protein (0.0206016). ECHS1, PECR

F.6. Endoplasmic Reticulum Stress Pathway (Enrichment Ratio* $\left.^{*} \log 10(p)\right)$.

Control Protein (0).

$3 x T g A D$ Protein $(\mathbf{0 . 0 4 7 8 4 1 )}$. EIF2AK3, CASP7.

F.7. Apoptosis Signaling (Enrichment Ratio* $-\log 10(p))$.

Control Protein (0).

$3 x T g A D$ Protein (0.059156). ACIN1, ROCK1, CAPN5, CAPN6, CAPN1, BAX, CASP7, PARP1.

F.8. Hypoxia Signaling (Enrichment Ratio* $-\log 10(p)$ ).

Control protein (0).

$3 x T g A D$ Protein (0.111983). VEGFA, P4HB, HSP90AB1, UBE2L3, NOS3, UBC, ARNT, ATM.

F.9. PPAR $\alpha / R X R \alpha$ Activation (Enrichment Ratio* $-\log 10(p))$.

Control Protein (0.0315615). PDIA3, PRKAR2A, NR2C2, SHC1, PRKAR2B, TGFB1, FASN, PRKACA, PLCB1, GOT2, PLCL1, PRKCB, PRKCA, PRKAR1A.

3xTgAD Protein (0.14586). PPARA, ACVR1, PRKAR2A, CD36, IL6, JAK2, ABCA1, IL1R2, ACADL, HSP90AB1, TGFB1, IRS1, PRKACA, SMAD4, IL1B, ADCY8, PLCD4, PRKAR1A.

\section{G.}

Receptor signaling pathway clusters of extracted proteins from primary cortical tissue. Specific receptor signaling pathway clusters generated in an un-biased manner using
Ingenuity Pathway Analysis (v. 8.5). The relative score generated for the degree of pathway population by proteins from the respective input sets (control or 3xTgAD) is shown in bold. The following are different types of signaling pathways.

\section{G.1. Dopamine Receptor Signaling.}

Control Protein (0.65824). PPP2R2A, PRKAR2A, PPP1R14B, PPP2CB, PPP2R1A, PRKAR2B, PPP1R10, PPP1R14D, PPP1R7, PPP2R2B, PRKACA, FREQ, PPP2R5E, PPP2R1B, PRKAR1A, CALY.

3xTgAD Protein (0.071199). PPP2R1A, FREQ, PRKAR2A, PRKACA, ADCY8, CALY, MAOA, PRKAR1A.

\section{G.2. PDGF Signaling.}

Control Protein (0.73892). SRC, CRKL, CRK, STAT3, FOS, SHC1, PIK3CG, CSNK2A1, PDGFRA, CAV1, CSNK2B, ELK1, STAT1, PRKCB, PRKCA.

3xTgAD Protein (0.15738). MYC, JAK1, PIK3C3, STAT3, PIK3R2, JAK2, ELK1, INPP5D.

\section{G.3. EGF Signaling.}

Control Protein (0.7956). SHC1, FOS, PIK3CG, CSNK2A1, EGF, CSNK2B, STAT3, STAT1, ELK1, PRKCA, EGFR.

$3 x T g A D$ Protein (0.22499). JAK1, PIK3C3, PIK3CB, STAT3, PIK3R2, ELK1, EGFR.

G.4. Neuregulin Signaling.

Control Protein (0.68068). SRC, PRKCQ, CRKL, EGF, CRK, ERBB3, PRKCZ, SHC1, PRKCI, PTPN11, ERBB4, CDKN1B, ELK1, PRKD1, PRKCB, EGFR, PRKCA.

3xTgAD Protein (0.1276). MYC, STAT5A, MTOR, PTPN11, HSP90AB1, PRKCD, PIK3R2, CDKN1B, ELK1, EGFR.

\section{G.5. Glutamate Receptor Signaling.}

Control Protein (0.53342). GRM5, GRIN2B, GRIN2A, GRIK4, GRIA1, GRID2, CALM2, GLUL, GRIP1, GRM4, GNG7, GRIK1.

$3 x \operatorname{TgAD}$ Protein (0).

G.6. Sonic Hedgehog Signaling.

Control Protein (0.73272). SHH, CCNB1, PRKAR2B, PRKAR2A, PRKACA, SMO, GLI1, PRKAR1A. 
3xTgAD Protein (0.20769). ADRBK1, PTCH1, PRKAR2A, PRKACA, PRKAR1A.

\section{G.7. TR/RXR Activation.}

Control Protein (0.5481). UCP2, RCAN2, UCP1, THRA, GRIP1, PFKP, PCK1, KLF9, SREBF1, DIO1, FASN, PIK3CG, STRBP, THRB, FGA, SYT12.

3xTgAD Protein (0.0321142). MTOR, PIK3C3, STRBP, ACACA, PIK3CB, PIK3R2, THRB.

\section{G.8. Ephrin Receptor Signaling.}

Control Protein (0.62472). GRIN2A, CDC42, EGF, CRK, GNG7, PGF, VEGFA, PTK2, SHC1, PAK1, SDC2, PIK3CG, EFNB1, EPHA7, GRIN2B, SRC, PAK2, CFL1, CRKL, EPHA3, STAT3, WIPF1, SDCBP, PTPN11, PAK3, EPHA5, EPHB3.

3xTgAD Protein (0.21037). FYN, PXN, CFL1, CDC42, KALRN, GNB2L1, GNAI1, RAC1, JAK2, STAT3, GNAZ, ROCK1, VEGFA, GNAI2, ROCK2, ABI1, PTPN11, PAK3, RHOA, GNAO1.

G.9. G-Protein Coupled Receptor Signaling.

Control Protein (0.3808). PDE7A, PDE4A, PDE1A, PDE4D, SHC1, SYNGAP1, PDE7B, PIK3CG, PLCB1, PRKCA, SRC, GRK4, EDNRB, PDE10A, PRKAR2A, PDE4B, STAT3, GRM4, PDE1C, CHRM5, GRM5, OPRD1, PRKAR2B, PRKACA, PRKCB, PRKAR1A.

3xTgAD Protein (0.13938). FYN, PTK2B, HTR4, ADRBK1, PDE10A, RGS7, PRKAR2A, GNAI1, RGS4, STAT3, PDE4B, GNAI2, HTR2C, PIK3C3, GNAO1, PRKACA, PIK3CB, PIK3R2, ADCY8, PRKAR1A.

G.10. Chemokine Signaling.

Control Protein (0.23961). PTK2, NOX1, SRC, FOS, CCL4, CFL1, PIK3CG, CALM2, PLCB1, PRKCA, PRKCB.

$3 x T g A D$ Protein (0.03232). ROCK2, GNAI2, CFL1, PTK2B, RHOA, GNAI1.

\section{G.11. Ceramide Signaling.}

Control Protein (0.15748). CTSD, PPP2CB, FOS, PPP2R1A, PPP2R2A, PIK3CG, PPP2R2B, PPP2R5E, PPP2R1B, PRKCZ.

$3 x \operatorname{TgAD}$ protein $(\mathbf{0})$.

G.12. FGF (Fibroblast Growth Factor) Signaling.
Control Protein (0.17423). FGFR3, PTPN11, FGF18, CRKL, PIK3CG, FGFR1, FGF23, FGFR2, CRK, STAT3, PRKCA.

3xTgAD Protein (0.0667352). PTPN11, PIK3C3, HGF, RAC1, PIK3CB, STAT3, PIK3R2, MAPKAPK2.

\section{G.13. Toll-like Receptor Signaling.}

Control Protein (0.1644). TLR4, FOS, TLR5, TLR6, TLR3, ELK1, TLR9.

3xTgAD Protein (0.065562). PPARA, TLR5, MAP3K7IP2, LBP, ELK1.

G.14. Nitric Oxide Signaling.

Control Protein (0.41157). GUCY1B2, GUCY1A3, GUCY2D, CALM2, PRKAR2A, PGF, VEGFA, PRKAR2B, PIK3CG, GUCY1A2, CAV1, PRKACA, PRKAR1A.

$3 x T g A D$ Protein (0.36801). VEGFA, HSP90AB1, GUCY2D, PRKCD, PIK3C3, GUCY2F, PRKAR2A, PRKACA, PIK3CB, PIK3R2, NOS3, PRKAR1A.

G.15. cAMP-Mediated Signaling.

Control Protein (0.39295). GRK4, SRC, CNGA4, PDE7A, PDE10A, PRKAR2A, CALM2, PDE4A, PPP3CC, STAT3, PDE4B, GRM4, PDE1A, PDE4D, CNGA1, PDE1C, CHRM5, OPRD1, PRKAR2B, PDE7B, PRKACA, PKIA, PRKAR1A.

3xTgAD Protein (0.40848). AKAP12, HTR4, ADRBK1, PDE10A, RGS7, PRKAR2A, GNAI1, RGS4, AKAP3, PPP3CC, STAT3, PDE4B, CNGA1, GNAI2, AKAP4, GNAO1, PRKACA, CNGB1, PKIA, ADCY8, AKAP9, PRKAR1A.

G.16. TGF- $\beta$ Signaling.

Control protein (0).

$3 x T g A D$ Protein (0.0236421). TGFB1, ACVR1, SMAD4, HNF4A, UBC, INHBC.

G.17. Neurotrophin/TRK Signaling.

Control Protein (0.016851). SHC1, FOS, CDC42, PTPN11, PIK3CG.

3xTgAD Protein (0.0440592). NTRK2, CDC42, PTPN11, PIK3C3, PIK3CB, PIK3R2.

G.18. IGF-1 Signaling. 
Control Protein (0.49715). PRKAR2A, PTK2, SHC1, FOS, PRKAR2B, PTPN11, PIK3CG, PRKACA, CSNK2A1, CSNK2B, ELK1, PRKAR1A.

3xTgAD Protein (0.52722). PXN, YWHAH, PRKAR2A, NEDD4, YWHAQ, PTPN11, IRS1, PIK3C3, PRKACA, IGF1R, IRS2, PIK3CB, PIK3R2, ELK1, PRKAR1A.

G.19. Serotonin Receptor Signaling.

Control protein (0).

3xTgAD Protein (0.059769). HTR2C, HTR4, HTR3A, MAOA.

G.20. Glucocorticoid Receptor Signaling.

Control protein (0).

$3 x T g A D$ Protein (0.066). STAT5A, JAK1, YWHAH, HSPA14, RAC1, IL6, PPP3CC, STAT3, JAK2, NFKB1, MNAT1, IL1R2, HSP90AB1, TGFB1, PIK3C3, CEBPA, PRKACA, SMAD4, IL1B, PIK3CB, PIK3R2, ELK1.

G.21. Aryl Hydrocarbon Receptor Signaling.

Control Protein (0.1218). SRC, CDK6, ALDH1L1, GSTO1, TGM2, CTSD, FOS, GSTM2, TGFB1, FASN, CDKN1B, ESR2, CDK2, FASLG, ATM, GSTK1.

3xTgAD Protein (0.31125). CYP1A1, NFIX, GSTM3, IL6, BAX, NFKB1, ARNT, MYC, AHRR, GSTM2, CCND2, HSP90AB1, TGFB1, ALDH1A3, IL1B, CDKN1B, AHR, MCM7, ATM.

\section{G.22. Insulin Receptor Signaling.}

Control Protein (0.28288). TSC1, TRIP10, CRKL, PRKAR2A, CRK, VAMP2, PPP1R14B, PRKCZ, SHC1, PRKCI, PPP1R10, PTPN11, PIK3CG, TSC2, PRKACA.

3xTgAD Protein (0.5056). TSC1, FYN, JAK1, PRKAR2A, JAK2, VAMP2, INPP5D, MTOR, PTPN11, IRS1, PIK3C3, PRKACA, EIF2B1, PIK3CB, IRS2, PIK3R2, PRKAR1A.

\section{G.23. Notch Signaling.}

Control Protein (0.0437248). DLL1, CNTN1, NCSTN, DLL3.

3xTgAD Protein (0.30609). NOTCH4, DLL1, NOTCH2, NCSTN, JAG1, NOTCH1, HEY1.

G.24. GABA Receptor Signaling.
Control Protein (0.099214). GABRG2, UBQLN1, GABBR1, GABARAP, UBC, GABRA3.

3xTgAD Protein (0.4216). DNM1, NSF, SLC32A1, UBQLN1, GABBR1, MYO5B, GABRE, UBC, GABRA3.

G.25. VEGF Signaling.

Control Protein (0.0252652). VEGFA, PTK2, SHC1, PIK3CG, PGF, PRKCA, PRKCB.

3xTgAD Protein (0.53464). EIF2S2, PXN, PTK2B, ACTB, NOS3, ARNT, ROCK2, VEGFA, ROCK1, PIK3C3, EIF2B1, PIK3CB, PIK3R2, ACTN4, ACTA1.

G.26. Integrin Signaling.

Control protein (0).

$3 x \operatorname{TgAD}$ Protein (0.54604). RAP2B, FYN, RALA, CDC42, TSPAN3, ARF6, RHOB, PIK3C3, ARF4, PIK3R2, ACTA1, CAPN5, CAPN6, PXN, ACTB, RALB, RAC1, ITGA6, RHOJ.

\section{H.}

Heatmap Protein Position Key. The individual position numbers are correlated to the specific multidimensional proteins represented by colored blocks in Figure 6. The following are the symbols of the proteins (the numbers between brackets refer to the position).

(1) adcyap1: adenylate cyclase activating polypeptide 1 (pituitary)

(2) adcyap1r1: adenylate cyclase activating polypeptide 1 (pituitary) receptor type I

(3) hap 1: huntingtin-associated protein 1

(4) htr4: 5-hydroxytryptamine (serotonin) receptor 4

(5) ntrk2: neurotrophic tyrosine kinase, receptor, type 2

(6) Kalrn: kalirin, RhoGEF kinase

(7) klc1: kinesin light chain 1

(8) adrbk1: adrenergic, beta, receptor kinase 1

(9) agrn: agrin

(10) apod: apolipoprotein D

(11) app: amyloid beta (A4) precursor protein

(12) arhgef11: Rho guanine nucleotide exchange factor (GEF) 11

(13) arpp 19: cAMP-regulated phosphoprotein, $19 \mathrm{kDa}$

(14) bacel: beta-site APP-cleaving enzyme 1

(15) ccs: copper chaperone for superoxide dismutase

(16) chgb: chromogranin B (secretogranin 1)

(17) flot1: flotillin 1

(18) flot2: flotillin 2 
(19) gnb211: guanine nucleotide binding protein ( $\mathrm{G}$ protein), beta polypeptide 2-like 1

(20) hyoul: hypoxia up-regulated 1

(21) lphn1: latrophilin 1

(22) lphn2: latrophilin 2

(23) magi3: membrane associated guanylate kinase, WW and PDZ domain containing 3

(24) nefh: neurofilament, heavy polypeptide

(25) prdx6: peroxiredoxin 6

(26) pscd2: cytohesin 2

(27) rtn3: reticulon 3

(28) rtn4r: reticulon 4 receptor

(29) slc44a4: solute carrier family 44, member 4

(30) stxbp1: syntaxin binding protein 1

(31) abca1: ATP-binding cassette, sub-family A (ABC1), member 1

(32) abil: abl-interactor 1

(33) adcy1: adenylate cyclase 1 (brain)

(34) adcy5: adenylate cyclase 5

(35) akap9: A kinase (PRKA) anchor protein 9

(36) aldh1a3: aldehyde dehydrogenase 1 family, member A3

(37) alox5: arachidonate 5-lipoxygenase

(38) arf6: ADP-ribosylation factor 6

(39) arhgef12: Rho guanine nucleotide exchange factor (GEF) 12

(40) atcay: ataxia, cerebellar, Cayman type

(41) atp2b1: ATPase, Ca++ transporting, plasma membrane 1

(42) atxn3: ataxin 3

(43) begain: brain-enriched guanylate kinase-associated homolog (rat)

(44) cabc1: chaperone, ABC1 activity of bcl complex homolog (S. pombe)

(45) capn1: calpain 1, (mu/l) large subunit

(46) caskin1: CASK interacting protein 1

(47) casp7: caspase 7, apoptosis-related cysteine peptidase

(48) cd36: CD36 molecule (thrombospondin receptor)

(49) cfd: complement factor D (adipsin)

(50) clu: clusterin

(51) cpa2: carboxypeptidase A2 (pancreatic)

(52) cript: cysteine-rich PDZ-binding protein

(53) dpp6: dipeptidyl-peptidase 6

(54) espn: espin

(55) fdps: farnesyl diphosphate synthase

(56) filip1: filamin A interacting protein 1
(57) gna11: guanine nucleotide binding protein (G protein), alpha 11 (Gq class)

(58) gnai2: guanine nucleotide binding protein (G protein), alpha inhibiting activity polypeptide 2

(59) gnao1: guanine nucleotide binding protein (G protein), alpha activating activity polypeptide $\mathrm{O}$

(60) gnaq: guanine nucleotide binding protein ( $\mathrm{G}$ protein), q polypeptide

(61) gnaz: guanine nucleotide binding protein ( $G$ protein), alpha $z$ polypeptide

(62) gpr56: G protein-coupled receptor 56

(63) hip1r: huntingtin interacting protein 1 related

(64) hmgcr: 3-hydroxy-3-methylglutaryl-Coenzyme A reductase

(65) hsf1: heat shock transcription factor 1

(66) htr2c: 5-hydroxytryptamine (serotonin) receptor 2C

(67) itm2c: integral membrane protein $2 \mathrm{C}$

(68) itpr3: inositol 1,4,5-triphosphate receptor, type 3

(69) lsamp: limbic system-associated membrane protein

(70) maoa: monoamine oxidase A

(71) map1lc3a: microtubule-associated protein 1 light chain 3 alpha

(72) mapkapk2: mitogen-activated protein kinase-activated protein kinase 2

(73) mark1: MAP/microtubule affinity-regulating kinase 1

(74) mdga2: MAM domain containing glycosylphosphatidylinositol anchor 2

(75) mpdz: multiple PDZ domain protein

(76) mpo: myeloperoxidase

(77) mpp7: membrane protein, palmitoylated 7 (MAGUK p55 subfamily member 7)

(78) ncam1: neural cell adhesion molecule 1

(79) ncam2: neural cell adhesion molecule 2

(80) ncdn: neurochondrin

(81) ndufs1: NADH dehydrogenase (ubiquinone) Fe-S protein $1,75 \mathrm{kDa}$ (NADH-coenzyme Q reductase)

(82) ndufs7: NADH dehydrogenase (ubiquinone) Fe-S protein 7, $20 \mathrm{kDa}(\mathrm{NADH}$-coenzyme $\mathrm{Q}$ reductase)

(83) nes: nestin

(84) nlgn2: neuroligin 2

(85) nlgn3: neuroligin 3

(86) npc2: Niemann-Pick disease, type C2

(87) npdc1: neural proliferation, differentiation and control, 1

(88) npy5r: neuropeptide Y receptor Y5

(89) nsf: N-ethylmaleimide-sensitive factor 
(90) padi2: peptidyl arginine deiminase, type II

(91) plcb4: phospholipase C, beta 4

(92) pxn: paxillin

(93) rab5a: RAB5A, member RAS oncogene family

(94) rgs4: regulator of $\mathrm{G}$-protein signaling 4

(95) rgs7: regulator of G-protein signaling 7

(96) rims1: regulating synaptic membrane exocytosis 1

(97) rora: RAR-related orphan receptor A

(98) sh3gl1: SH3-domain GRB2-like 1

(99) sod2: superoxide dismutase 2, mitochondrial

(100) sst: somatostatin

(101) vldlr: very low density lipoprotein receptor

(102) camkk1: calcium/calmodulin-dependent protein kinase kinase 1 , alpha

(103) cacna2d2: calcium channel, voltage-dependent, alpha 2/delta subunit 2

(104) vcp: valosin-containing protein

(105) stx1a: syntaxin 1A (brain)

(106) vgf: VGF nerve growth factor inducible

(107) calb2: calbindin 2

(108) prdx1: peroxiredoxin 1

(109) stmn2: stathmin-like 2

(110) vsnl1: visinin-like 1

(111) cdh2: cadherin 2, type 1, N-cadherin (neuronal)

(112) dbn1: drebrin 1

(113) oxr1: oxidation resistance 1

(114) gpsm1: G-protein signaling modulator 1 (AGS3-like, C. elegans)

(115) gmfb: glia maturation factor, beta

(116) cd2ap: CD2-associated protein

(117) centa1: ArfGAP with dual PH domains 1

(118) ncstn: nicastrin

(119) pak3: p21 protein (Cdc42/Rac)-activated kinase 3

(120) sncb: synuclein, beta

(121) calb1: calbindin 1

(122) cnr1: cannabinoid receptor 1 (brain)

(123) disc1: disrupted in schizophrenia 1

(124) dlgap2: discs, large (Drosophila) homolog-associated protein 2

(125) dlgap4: discs, large (Drosophila) homolog-associated protein 4

(126) dtnb: dystrobrevin, beta

(127) dvl1: dishevelled, dsh homolog 1 (Drosophila)

(128) exoc7: exocyst complex component 7

(129) gabbr1: gamma-aminobutyric acid (GABA) B receptor, 1
(130) panxl: pannexin 1

(131) pclo: piccolo (presynaptic cytomatrix protein)

(132) phactr3: phosphatase and actin regulator 3

(133) picalm: phosphatidylinositol binding clathrin assembly protein

(134) shank1: SH3 and multiple ankyrin repeat domains 1

(135) snap91: synaptosomal-associated protein, $91 \mathrm{kDa}$ homolog (mouse)

(136) stmn4: stathmin-like 4

(137) sod1: superoxide dismutase 1, soluble

(138) synj2: synaptojanin 2

(139) tub: tubby homolog (mouse)

(140) snca: synuclein, alpha (non A4 component of amyloid precursor)

(141) park7: Parkinson disease (autosomal recessive, early onset) 7

(142) pcsk1n: proprotein convertase subtilisin/kexin type 1 inhibitor

(143) palm: Paralemmin

(144) vapb: VAMP (vesicle-associated membrane protein)associated protein $\mathrm{B}$ and $\mathrm{C}$

(145) cttn: cortactin

(146) cacnala: calcium channel, voltage-dependent, P/Q type, alpha 1A subunit

(147) celsr3: cadherin, EGF LAG seven-pass G-type receptor 3 (flamingo homolog, Drosophila)

(148) chl1: cell adhesion molecule with homology to L1CAM (close homolog of L1)

(149) chrm5: cholinergic receptor, muscarinic 5

(150) cnksr2: connector enhancer of kinase suppressor of Ras 2

(151) cnp: $2^{\prime}, 3^{\prime}$-cyclic nucleotide $3^{\prime}$ phosphodiesterase

(152) cntfr: ciliary neurotrophic factor receptor

(153) cntn1: contactin 1

(154) cplx1: complexin 1

(155) dag1: dystroglycan 1 (dystrophin-associated glycoprotein 1)

(156) dbndd2: dysbindin (dystrobrevin binding protein 1) domain containing 2

(157) dbnl: drebrin-like

(158) dclk2: doublecortin-like kinase 2

(159) dlgap1: discs, large (Drosophila) homolog-associated protein 1

(160) dscam: Down syndrome cell adhesion molecule

(161) ecel1: endothelin converting enzyme-like 1

(162) efnb1: ephrin-B1

(163) eml5: echinoderm microtubule associated protein like 5 
(164) epha3: EPH receptor A3

(165) epha5: EPH receptor A5

(166) epha7: EPH receptor A7

(167) ephb3: EPH receptor B3

(168) erbb4: v-erb-a erythroblastic leukemia viral oncogene homolog 4 (avian)

(169) evl: Enah/Vasp-like

(170) exoc5: exocyst complex component 5

(171) fzd9: frizzled homolog 9 (Drosophila)

(172) GABARAP: GABA(A) receptor-associated protein

(173) gabarapl2: GABA(A) receptor-associated proteinlike 2

(174) gabrr3: gamma-aminobutyric acid (GABA) A receptor, alpha 3

(175) gcgr: glucagon receptor

(176) gng7: guanine nucleotide binding protein ( $\mathrm{G}$ protein), gamma 7

(177) got1: glutamic-oxaloacetic transaminase 1, soluble (aspartate aminotransferase 1)

(178) got2: glutamic-oxaloacetic transaminase 2, mitochondrial (aspartate aminotransferase 2)

(179) gprc6a: G protein-coupled receptor, family C, group 6 , member A

(180) gria1: glutamate receptor, ionotropic, AMPA 1

(181) grid2: glutamate receptor, ionotropic, delta 2

(182) grik4: glutamate receptor, ionotropic, kainate 4

(183) grin2a: glutamate receptor, ionotropic, N-methyl Daspartate $2 \mathrm{~A}$

(184) grip1: glutamate receptor interacting protein 1

(185) gripap1: GRIP1 associated protein 1

(186) grk6: G protein-coupled receptor kinase 6

(187) opa1: optic atrophy 1 (autosomal dominant)

(188) pak1: p21 protein (Cdc42/Rac)-activated kinase 1

(189) pak2: p21 protein (Cdc42/Rac)-activated kinase 2

(190) pawr: PRKC, apoptosis, WT1, regulator

(191) phactr1: phosphatase and actin regulator 1

(192) pip5k1c: phosphatidylinositol-4-phosphate 5-kinase, type I, gamma

(193) plcb1: phospholipase C, beta 1 (phosphoinositidespecific)

(194) pld3: phospholipase D family, member 3

(195) ppfia3: protein tyrosine phosphatase, receptor type, $\mathrm{f}$ polypeptide (PTPRF), interacting protein (liprin), alpha 3

(196) ppid: peptidylprolyl isomerase D

(197) ppp2cb: protein phosphatase 2 (formerly 2A), catalytic subunit, beta isoform
(198) ppp2r2a: protein phosphatase 2 (formerly $2 \mathrm{~A}$ ), regulatory subunit $\mathrm{B}$, alpha isoform

(199) prkar2b: protein kinase, cAMP-dependent, regulatory, type II, beta

(200) prnp: prion protein

(201) ptk2: PTK2 protein tyrosine kinase 2

(202) ptn: pleiotrophin

(203) ryr3: ryanodine receptor 3

(204) sdcbp: syndecan binding protein (syntenin)

(205) sh3gl3: SH3-domain GRB2-like 3

(206) shank2: SH3 and multiple ankyrin repeat domains 2

(207) sirt2: sirtuin (silent mating type information regulation 2 homolog) 2 (S. cerevisiae)

(208) smu1: smu-1 suppressor of mec-8 and unc-52 homolog (C. elegans)

(209) soat1: sterol O-acyltransferase 1

(210) sstr2: somatostatin receptor 2

(211) ssx2ip: synovial sarcoma, $\mathrm{X}$ breakpoint 2 interacting protein

(212) stau1: staufen, RNA binding protein, homolog 1 (Drosophila)

(213) stmn3: stathmin-like 3

(214) strn: striatin, calmodulin binding protein

(215) syn1: synapsin I

(216) syngap1: synaptic Ras GTPase activating protein 1 homolog (rat)

(217) synj2bp: synaptojanin 2 binding protein

(218) synpo: synaptopodin

(219) syt11: synaptotagmin XI

(220) trap1: TNF receptor-associated protein 1

(221) trpc2: transient receptor potential cation channel, subfamily C, member 2 (pseudogene)

(222) trpc4: transient receptor potential cation channel, subfamily $\mathrm{C}$, member 4

(223) trpc5: transient receptor potential cation channel, subfamily $\mathrm{C}$, member 5

(224) wnt7a: wingless-type MMTV integration site family, member 7A

(225) tuba1a: tubulin, alpha 1a

(226) vipr2: vasoactive intestinal peptide receptor 2

(227) trak2: trafficking protein, kinesin binding 2

(228) snx17: sorting nexin 17

(229) psd: pleckstrin and Sec7 domain containing

(230) ppp2r2b: protein phosphatase 2 (formerly $2 \mathrm{~A}$ ), regulatory subunit $\mathrm{B}$, beta isoform

(231) ppp1r9b: protein phosphatase 1, regulatory (inhibitor) subunit $9 \mathrm{~B}$ 
(232) grm5: glutamate receptor, metabotropic 5

(233) grk5: G protein-coupled receptor kinase 5

(234) grin2b: glutamate receptor, ionotropic, N-methyl Daspartate $2 \mathrm{~B}$

(235) grik1: glutamate receptor, ionotropic, kainate 1

(236) grasp: GRP1 (general receptor for phosphoinositides 1)-associated scaffold protein

(237) gipc1: GIPC PDZ domain containing family, member 1

(238) dpysl4: dihydropyrimidinase-like 4

(239) dpysl3: dihydropyrimidinase-like 3

(240) dgkb: diacylglycerol kinase, beta $90 \mathrm{kDa}$

(241) ddn: dendrin

(242) cit: citron (rho-interacting, serine/threonine kinase 21)

(243) grm4: glutamate receptor, metabotropic 4

(244) ctnnd2: catenin (cadherin-associated protein), delta 2 (neural plakophilin-related arm-repeat protein).

I.

Receptor-specific protein interaction networks in lipid raft extracts from control animals. IPA-generated receptorspecific protein lists from control lipid raft samples were clustered into coherent functional interaction networks. Focus molecules (BOLD in "molecules in network") denote the proteins that are present in the predicted reaction network as well as the experimental input protein set. The following are different types of interaction networks.

(1) Control: cell signaling, nucleic acid metabolism, small molecule biochemistry; score: 32; focus molecule: 15; molecules in network: ADCY, Akt, Beta-Arrestin, CD4, CHRM5, CNTFR, Creb, EDNRB, ERK1/2, FSHR, GABBR1, GCGR, Gpcr, GPR3, GPR12, GPR20, GPR34, GPR65, GPR161, GRM5, hCG, Mapk, OPRD1, OVGP1, P2RY6, P2RY11, PDGF BB, PI3K, Pkc(s), PTGER2, SFRP4, SSTR2, THBD, UNC5B, VIPR2.

(2) Control: infectious disease, antigen presentation, antimicrobial response; score: 31; focus molecule: 15; molecules in network: Ap1, CHRNB3, CHRNB4, CHRNG, CLDN4, CNR1, CXADR, G alphai, Ifn, IFN Beta, IFN TYPE 1, IgG, IKK (complex), IL-1R/TLR, IL12 (complex), Il12 (family), Interferon alpha, IRAK, IRF, NFkB (complex), NRG, OSMR, P38 MAPK, SMO, TACR1, Tlr, TLR3, TLR4, TLR5, TLR6, TLR9, TSHR, Ubiquitin.

(3) Control: cellular development tumor morphology, cell death; score: 18; focus molecule: 10; molecules in network: APOL5, ARR3, ARTN, ASB16, Cadherin (E,N,P,VE), CELSR2, CELSR3, CTNNB1, CTNN $\beta-C D H E / N$, DAG1, EVX1, FZD9, GDNF, GFRA1, GFRA3, GLTSCR1, GPRC6A, GRB2, GRM4, HRH4, KCNN1, OPN1LW, OPN1SW, PALM2-AKAP2, PHACTR2, PRICKLE3, PTH2R, SAG, SEPN1, SHROOM2, SRC.
(4) Control: carbohydrate metabolism; score: 2; focus molecule: 1; molecules in network: CLEC4A, IL13.

(5) Control: cell-to-cell signaling and interaction, nervous system development and function; score: 2; focus molecule: 1; molecules in network: PRPH2, ROM1.

(6) Control: protein synthesis, molecular transport, protein trafficking; score: 2; focus molecule: 1; molecules in network: GABRR3, PRKCZ, SQSTM1.

\section{J.}

Receptor-specific protein interaction networks in lipid raft extracts from 3xTgAD animals. IPA-generated receptorspecific protein lists from $3 x$ TgAD lipid raft samples were clustered into coherent functional interaction networks. Focus molecules (BOLD in "molecules in network") denote the proteins that are present in the predicted reaction network as well as the experimental input protein set. The following are different types of interaction networks.

(1) 3xTgAD: metabolic disease, endocrine system disorders, cell signaling; score: 45; focus molecule: 18; molecules in network: ADCYAP1R1, Ap1, CD36, CD86, CNR1, Creb, CREB-NFkB, CXADR, ERK, ERK1/2, GABBR1, GPR56, hCG, HTR4, HTR2C, IGF1R, IGF2R, IgG, IL12 (complex), IL1R2, Insulin, Jnk, LDL, LIFR, LRPAP1, Mapk, MIP1, NFkB (complex), NPR2, OVGP1, P38 MAPK, Pkc(s), TLR5, TNFRSF14, TSHR.

(2) 3xTgAD: cell signaling, nucleic acid metabolism, small molecule biochemistry; score: 13; focus molecule: 7; molecules in network: AATK, ABR, AKR1A1, BPI, CACNG5, CRYM, cyclic AMP, CYP26B1, DHRS3, DLG4, GH1, Histone h3, HSD17B11, KCTD11, KIF3C, LAP3, LPHN1, LPHN2, OPN1LW, OPN1SW, P2RY11, PTCH1, PTH2R, RLN3, RN5S, ROBO1, ROS1, RXFP1, RXFP2, SERPINB8, TMEM49, TNF, USP3.

(3) 3xTgAD: cell signaling, cellular function and maintenance, molecular transport; score: 2; focus molecule: 1; molecules in network: CFTR, FREQ, IL1RAPL1, MYD88.

(4) 3xTgAD: cell-to-cell signaling and interaction, cellular function and maintenance, cellular movement; score: 2; focus molecule: 1; molecules in network: GPR1, PAX3, PRDM5.

(5) 3XTgAD: behavior, digestive system development and function, cell morphology; score: 2; focus molecule: 1; molecules in network: NPY, NPY5R, PPY, PYY, SSB.

(6) 3xTgAD: cancer, reproductive system disease, gene expression; score: 2 ; focus molecule: 1 ; molecules in network: FOS, MIR103-1, MIR103-2, MIR107, MIRLET7G, MYC, OMG, RHOA, RTN4R.

\section{Acknowledgments}

This work was carried out with the support of the Intramural Research Program of the National Institute on Aging, National Institutes of Health. The authors thank Dr. LaFerla for kindly donating 3xTgAD breeding pairs to the National Institute on Aging. 


\section{References}

[1] J. W. Ashford, “APOE genotype effects on Alzheimer's disease onset and epidemiology," Journal of Molecular Neuroscience, vol. 23, no. 3, pp. 157-165, 2004.

[2] M. P. Mattson, S. Maudsley, and B. Martin, "BDNF and 5HT: a dynamic duo in age-related neuronal plasticity and neurodegenerative disorders," Trends in Neurosciences, vol. 27, no. 10, pp. 589-594, 2004.

[3] J. Hardy, K. Duff, K. G. Hardy, J. Perez-Tur, and M. Hutton, "Genetic dissection of Alzheimer's disease and related dementias: amyloid and its relationship to tau," Nature Neuroscience, vol. 1, no. 5, pp. 355-358, 1998.

[4] J. Hardy and D. J. Selkoe, "The amyloid hypothesis of Alzheimer's disease: progress and problems on the road to therapeutics," Science, vol. 297, no. 5580, pp. 353-356, 2002.

[5] S. Maudsley and M. P. Mattson, "Protein twists and turns in Alzheimer disease," Nature Medicine, vol. 12, no. 4, pp. 392393, 2006.

[6] R. W. Mahley, "Apolipoprotein E: cholesterol transport protein with expanding role in cell biology," Science, vol. 240, no. 4852, pp. 622-630, 1988.

[7] W. J. Strittmatter, A. M. Saunders, D. Schmechel et al., "Apolipoprotein E: high-avidity binding to $\beta$-amyloid and increased frequency of type 4 allele in late-onset familial Alzheimer disease," Proceedings of the National Academy of Sciences of the United States of America, vol. 90, no. 5, pp. 19771981, 1993.

[8] C.-L. Schengrund, "Lipid rafts: keys to neurodegeneration," Brain Research Bulletin, vol. 82, no. 1-2, pp. 7-17, 2010.

[9] D. Lingwood and K. Simons, "Lipid rafts as a membraneorganizing principle," Science, vol. 327, no. 5961, pp. 46-50, 2010.

[10] G. P. Gellermann, T. R. Appel, A. Tannert et al., "Raft lipids as common components of human extracellular amyloid fibrils," Proceedings of the National Academy of Sciences of the United States of America, vol. 102, no. 18, pp. 6297-6302, 2005.

[11] S.-I. Kubo, V. M. Nemani, R. J. Chalkley et al., "A combinatorial code for the interaction of $\alpha$-synuclein with membranes," Journal of Biological Chemistry, vol. 280, no. 36, pp. 3166431672, 2005.

[12] R. J. Morris, C. J. Parkyn, and A. Jen, "Traffic of prion protein between different compartments on the neuronal surface, and the propagation of prion disease," FEBS Letters, vol. 580, no. 23, pp. 5565-5571, 2006.

[13] D. R. Riddell, G. Christie, I. Hussain, and C. Dingwall, "Compartmentalization of $\beta$-secretase (Asp2) into low-buoyant density, noncaveolar lipid rafts," Current Biology, vol. 11, no. 16, pp. 1288-1293, 2001.

[14] M. O. W. Grimm, H. S. Grimm, I. Tomic, K. Beyreuther, T. Hartmann, and C. Bergmann, "Independent inhibition of Alzheimer disease $\beta$ - and $\gamma$-secretase cleavage by lowered cholesterol levels," Journal of Biological Chemistry, vol. 283, no. 17, pp. 11302-11311, 2008.

[15] L. Kalvodova, N. Kahya, P. Schwille et al., "Lipids as modulators of proteolytic activity of BACE: involvement of cholesterol, glycosphingolipids, and anionic phospholipids in vitro," Journal of Biological Chemistry, vol. 280, no. 44, pp. 36815-36823, 2005.

[16] N. Yamamoto, Y. Hirabayashi, M. Amari et al., "Assembly of hereditary amyloid $\beta$-protein variants in the presence of favorable gangliosides," FEBS Letters, vol. 579, no. 10, pp. 2185-2190, 2005.
[17] N. Yamamoto, K. Matsuzaki, and K. Yanagisawa, "Crossseeding of wild-type and hereditary variant-type amyloid $\beta$ proteins in the presence of gangliosides," Journal of Neurochemistry, vol. 95, no. 4, pp. 1167-1176, 2005.

[18] T. Ariga, M. P. McDonald, and R. K. Yu, "Role of ganglioside metabolism in the pathogenesis of Alzheimer's disease-a review," Journal of Lipid Research, vol. 49, no. 6, pp. 1157-1175, 2008.

[19] R. Williamson, A. Usardi, D. P. Hanger, and B. H. Anderton, "Membrane-bound $\beta$-amyloid oligomers are recruited into lipid rafts by a fyn-dependent mechanism," FASEB Journal, vol. 22, no. 5, pp. 1552-1559, 2008.

[20] K. Bhaskar, S.-H. Yen, and G. Lee, "Disease-related modifications in tau affect the interaction between Fyn and tau," Journal of Biological Chemistry, vol. 280, no. 42, pp. 3511935125, 2005.

[21] T. A. Fulga, I. Elson-Schwab, V. Khurana et al., "Abnormal bundling and accumulation of F-actin mediates tau-induced neuronal degeneration in vivo," Nature Cell Biology, vol. 9, no. 2, pp. 139-148, 2007.

[22] S. Oddo, A. Caccamo, J. D. Shepherd et al., "Triple-transgenic model of Alzheimer's Disease with plaques and tangles: intracellular A $\beta$ and synaptic dysfunction," Neuron, vol. 39, no. 3, pp. 409-421, 2003

[23] R. L. Nelson, Z. Guo, V. M. Halagappa et al., "Prophylactic treatment with paroxetine ameliorates behavioral deficits and retards the development of amyloid and tau pathologies in 3xTgAD mice," Experimental Neurology, vol. 205, no. 1, pp. 166-176, 2007.

[24] B. Martin, R. Brenneman, K. G. Becker, M. Gucek, R. N. Cole, and S. Maudsley, "iTRAQ analysis of complex proteome alterations in 3xTgAD Alzheimer's mice: understanding the interface between physiology and disease," PLoS ONE, vol. 3, no. 7, article e2750, 2008.

[25] A. M. Stranahan, K. Lee, K. G. Becker et al., "Hippocampal gene expression patterns underlying the enhancement of memory by running in aged mice," Neurobiology of Aging, vol. 31, no. 11, pp. 1937-1949, 2010.

[26] S. Maudsley, K. L. Pierce, A. M. Zamah et al., "The $\beta 2-$ adrenergic receptor mediates extracellular signal-regulated kinase activation via assembly of a multi-receptor complex with the epidermal growth factor receptor," Journal of Biological Chemistry, vol. 275, no. 13, pp. 9572-9580, 2000.

[27] S. E. Calvano, W. Xiao, D. R. Richards et al., "A network-based analysis of systemic inflammation in humans," Nature, vol. 437, no. 7061, pp. 1032-1037, 2005.

[28] B. Martin, M. P. Mattson, and S. Maudsley, "Caloric restriction and intermittent fasting: two potential diets for successful brain aging," Ageing Research Reviews, vol. 5, no. 3, pp. 332353,2006

[29] J. Murray and R. A. Capaldi, "Screening for the metabolic basis of neurodegeneration: developing a focused proteomic approach," Annals of the New York Academy of Sciences, vol. 1147, pp. 348-357, 2008.

[30] G. J. Brewer, "Epigenetic oxidative redox shift (EORS) theory of aging unifies the free radical and insulin signaling theories," Experimental Gerontology, vol. 45, no. 3, pp. 173-179, 2010.

[31] J. A. Allen, R. A. Halverson-Tamboli, and M. M. Rasenick, "Lipid raft microdomains and neurotransmitter signalling," Nature Reviews Neuroscience, vol. 8, no. 2, pp. 128-140, 2007.

[32] S. L. Petersen, S. Krishnan, and E. D. Hudgens, "The aryl hydrocarbon receptor pathway and sexual differentiation of neuroendocrine functions," Endocrinology, vol. 147, no. 6, pp. S33-S42, 2006. 
[33] J. Caltagarone, Z. Jing, and R. Bowser, "Focal adhesions regulate $\mathrm{A} \beta$ signaling and cell death in Alzheimer's disease," Biochimica et Biophysica Acta, vol. 1772, no. 4, pp. 438-445, 2007.

[34] S. Maudsley, B. Martin, and L. M. Luttrell, "G protein-coupled receptor signaling complexity in neuronal tissue: implications for novel therapeutics," Current Alzheimer Research, vol. 4, no. 1, pp. 3-19, 2007.

[35] S. Maudsley, B. Martin, and L. M. Luttrell, "The origins of diversity and specificity in $\mathrm{G}$ protein-coupled receptor signaling," Journal of Pharmacology and Experimental Therapeutics, vol. 314, no. 2, pp. 485-494, 2005.

[36] N. C. Inestrosa and E. Arenas, "Emerging roles of Wnts in the adult nervous system," Nature Reviews Neuroscience, vol. 11, no. 2, pp. 77-86, 2010.

[37] J. A. Johnson, D. A. Johnson, A. D. Kraft et al., "The Nrf2ARE pathway: an indicator and modulator of oxidative stress in neurodegeneration," Annals of the New York Academy of Sciences, vol. 1147, pp. 61-69, 2008.

[38] M. W. Marlatt and P. J. Lucassen, "Neurogenesis and Alzheimer's disease: biology and pathophysiology in mice and men," Current Alzheimer Research, vol. 7, no. 2, pp. 113-125, 2010.

[39] E. Cohen, J. F. Paulsson, P. Blinder et al., "Reduced IGF-1 signaling delays age-associated proteotoxicity in mice," Cell, vol. 139, no. 6, pp. 1157-1169, 2009.

[40] S. Freude, K. Schilbach, and M. Schubert, "The role of IGF-1 receptor and insulin receptor signaling for the pathogenesis of Alzheimer's disease: from model organisms to human disease," Current Alzheimer Research, vol. 6, no. 3, pp. 213-223, 2009.

[41] W. Araki, H. Kume, A. Oda, A. Tamaoka, and F. Kametani, "IGF-1 promotes $\beta$-amyloid production by a secretaseindependent mechanism," Biochemical and Biophysical Research Communications, vol. 380, no. 1, pp. 111-114, 2009.

[42] B. Martin, R. Brenneman, E. Golden et al., "Growth factor signals in neural cells: coherent patterns of interaction control multiple levels of molecular and phenotypic responses," Journal of Biological Chemistry, vol. 284, no. 4, pp. 2493-2511, 2009.

[43] Y. A. Ushkaryov, A. Rohou, and S. Sugita, "alpha-Latrotoxin and its receptors," Handbook of Experimental Pharmacology, vol. 184, pp. 171-206, 2008.

[44] J. O. Reilly, I. D. Karavanova, K. P. Williams, N. K. Mahanthappa, and K. L. Allendoerfer, "Cooperative effects of Sonic hedgehog and NGF on basal forebrain cholinergic neurons," Molecular and Cellular Neuroscience, vol. 19, no. 1, pp. 88-96, 2002.

[45] A. Thathiah and B. De Strooper, "G protein-coupled receptors, cholinergic dysfunction, and $\mathrm{A} \beta$ toxicity in Alzheimer's disease," Science Signaling, vol. 2, no. 93, p. re8, 2009. 


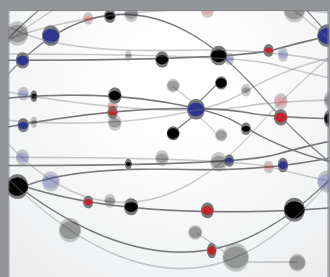

The Scientific World Journal
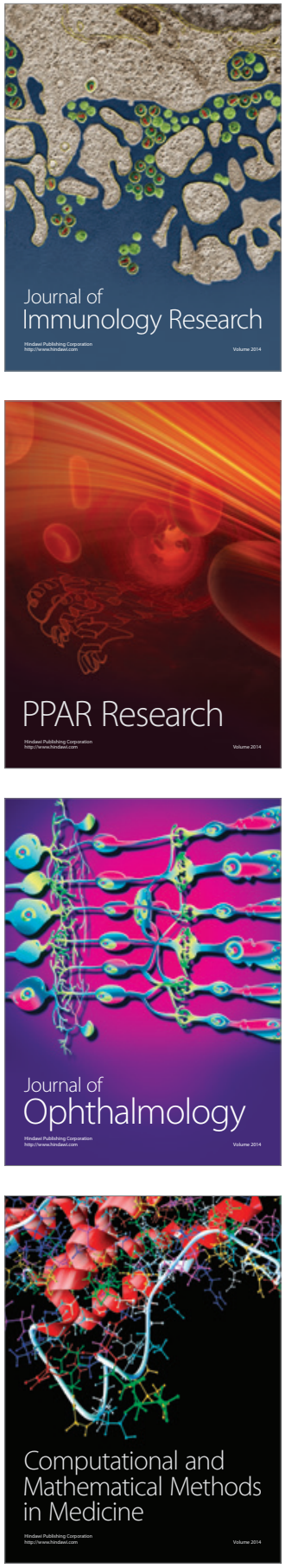

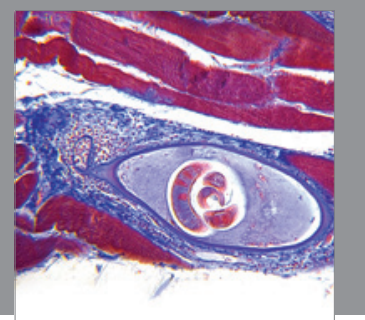

Gastroenterology

Research and Practice
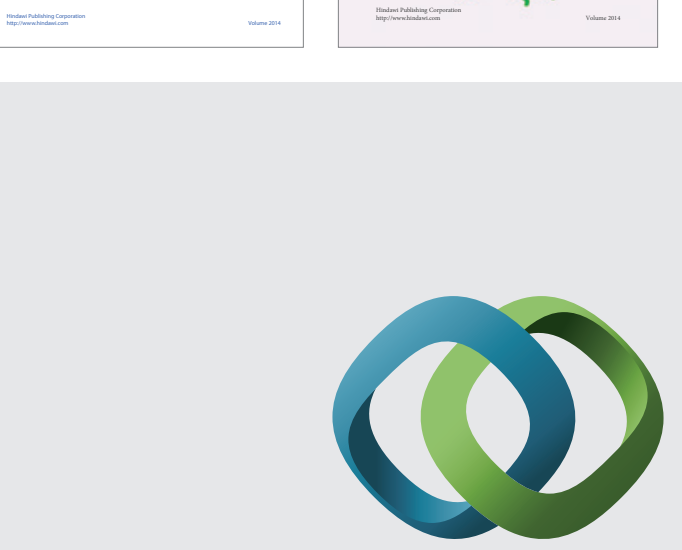

\section{Hindawi}

Submit your manuscripts at

http://www.hindawi.com
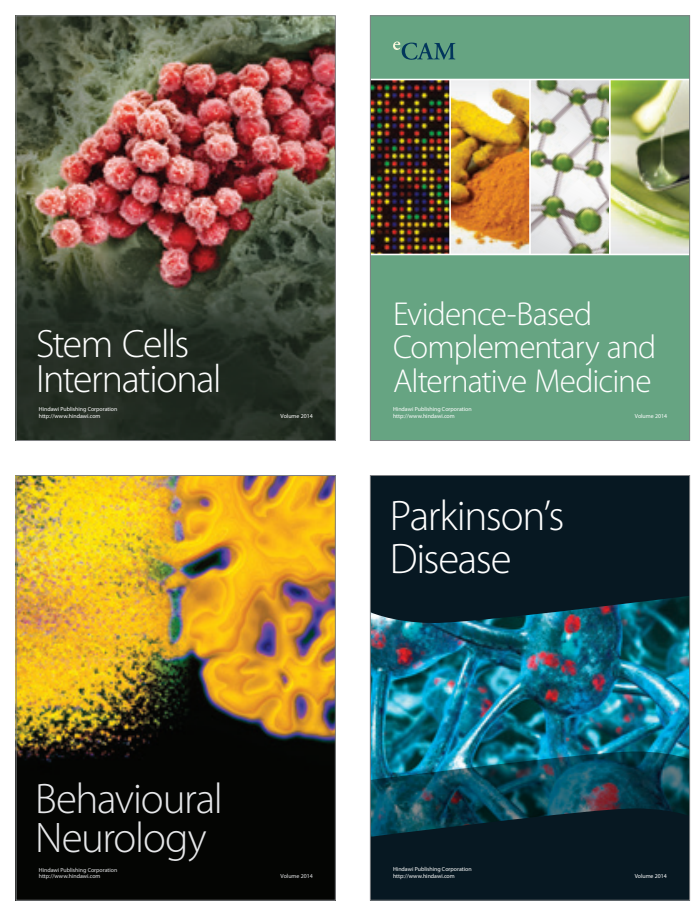

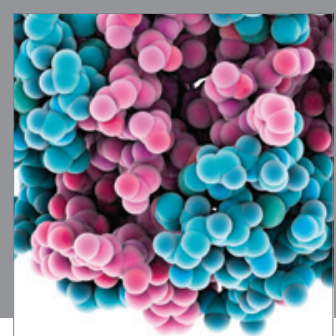

Journal of
Diabetes Research

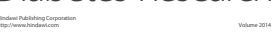

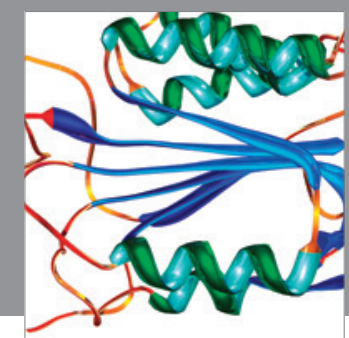

Disease Markers
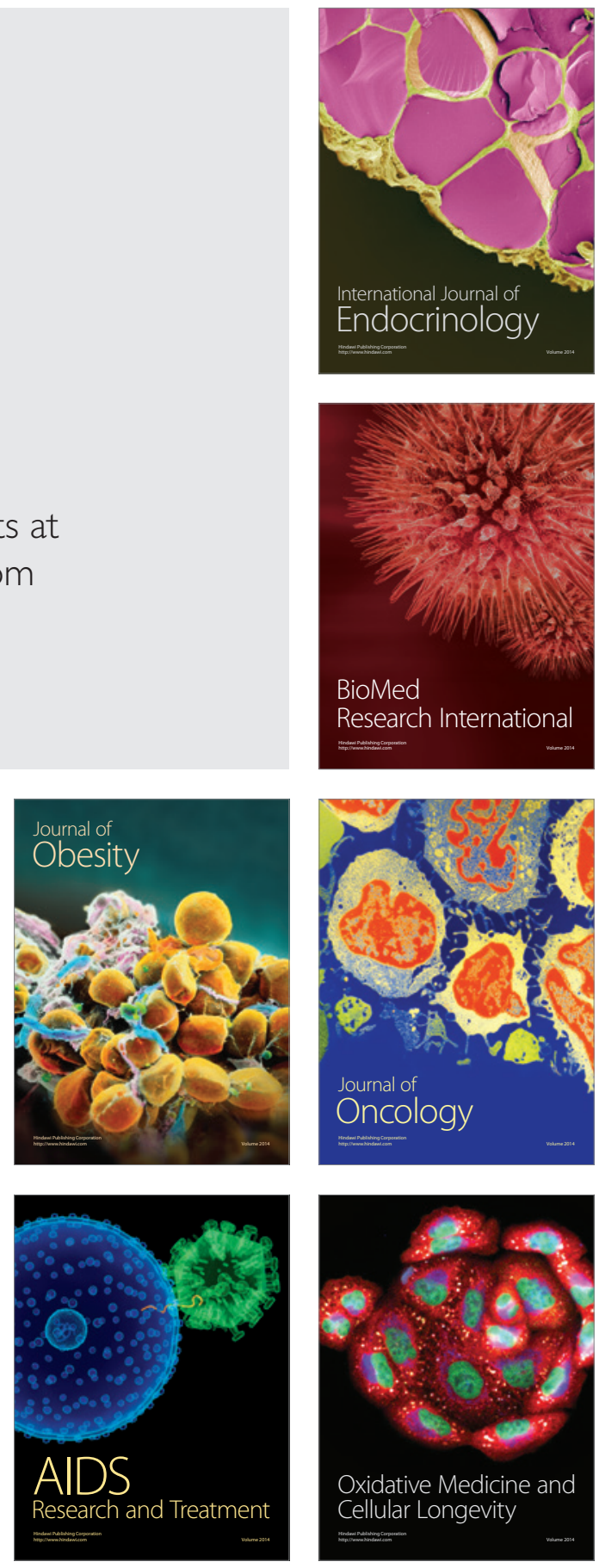